\title{
EQUATION DISCOVERY IN DATABASES FROM ENGINEERING
}

\author{
By \\ Liye Zhang \\ W. M. Kim Roddis \\ A Report on Research Sponsored by \\ THE NATIONAL SCIENCE FOUNDATION \\ Research Grant No. CDA-9401021 Amendment 4 \\ Proposal No. CDA-9743731 \\ INFORMATION \& TELECOMMUNICATION \\ TECHNOLOGY CENTER \\ University of Kansas
}

Structural Engineering and Engineering Materials SM Report No. 53 April 1999 


\title{
EQUATION DISCOVERY IN DATABASES FROM ENGINEERING
}

\author{
By \\ Liye Zhang \\ W. M. Kim Roddis
}

A Report on Research Sponsored by

THE NATIONAL SCIENCE FOUNDATION

Research Grant No. CDA-9401021 Amendment 4

Proposal No. CDA-9743731

\section{INFORMATION AND TELECOMMUNICATION TECHNOLOGY CENTER University of Kansas}

Structural Engineering and Engineering Materials SM Report No. 53

UNIVERSITY OF KANSAS CENTER FOR RESEARCH, INC.

LAWRENCE, KANSAS

April 1999 


\section{ABSTRACT}

As the quantity of electronically generated engineering data grows rapidly, building computer systems to analyze data automatically and intelligently becomes increasingly important to engineers. The overall process of extracting useable knowledge from electronically stored data is called knowledge discovery in databases. The part of the process where patterns are extracted or models are built is referred to as data mining.

This dissertation proposes a data mining method that combines machine learning and regression to help engineers in acquiring knowledge which is preferably expressed as equations. A learning algorithm based on the method has been implemented in the computer system EDDE (Equation Discovery in Databases from Engineering). In addition, to obtain useful models that are understandable to engineers, knowledge specific to the particular problem area is incorporated into EDDE to guide the discovery process. The role of this domain knowledge is investigated.

The system EDDE is extensively tested on both synthetic data sets and actual engineering data sets. The tests on synthetic data show that EDDE has some important features, such as not being sensitive to the number of variables in data sets. When compared to other methods (regression tree CART, instances based IBL, multivariate linear regression, model tree M5, neural nets, and combinations of these methods), EDDE generates a smaller size model with lower prediction error. EDDE thus summarizes the data more concisely and describes the data better.

EDDE has been used to analyze actual data sets from civil engineering (duration of construction activities, development/splice length of reinforcing bars, and 


\section{CONTENTS}

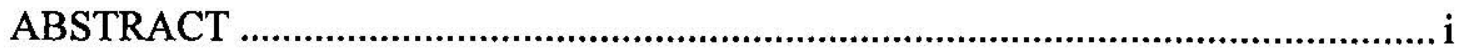

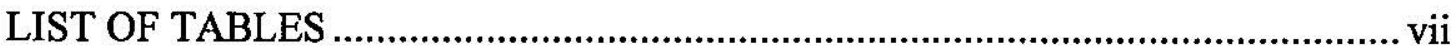

LIST OF FIGURES...................................................................................... viii

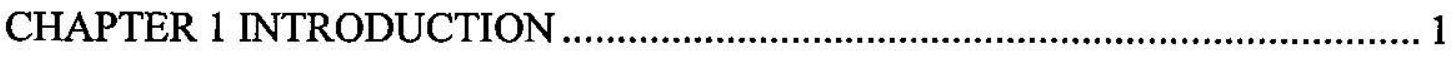

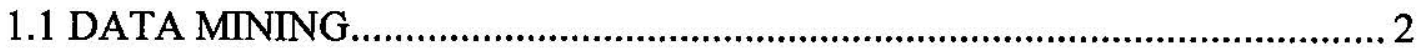

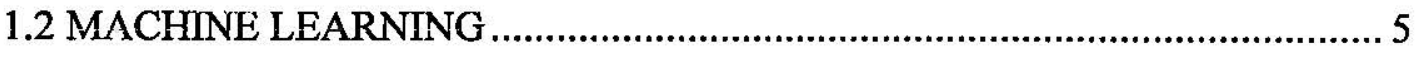

1.3 INTENDED PR.OBLEM CHARACTERISTICS ……...................................... 7

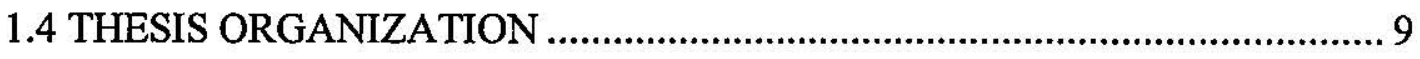

CHAPTER 2 REVIEW OF RELATED WORK ..................................................... 11

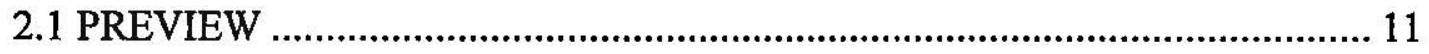

2.2 GENERAL REVIEW OF MACHINE LEARNING METHODS.................... 12

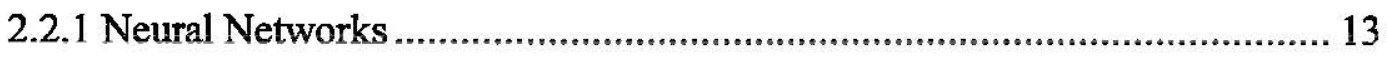

2.2.2 Instance-Based or Case-Based Methods ................................................... 13

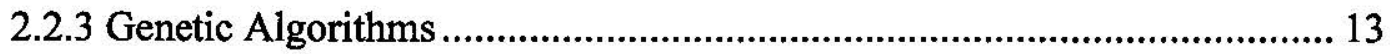

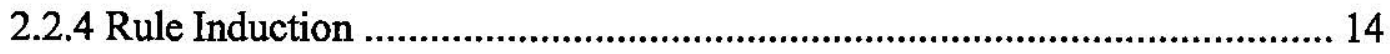

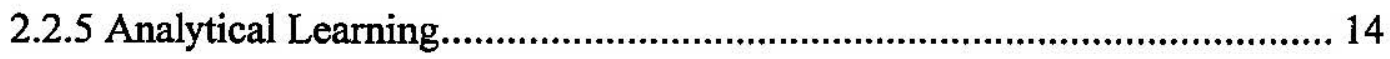

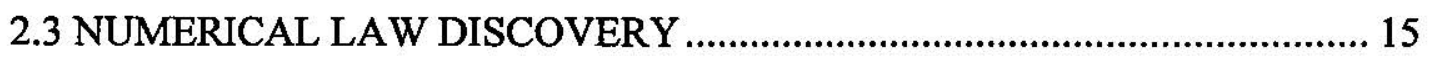

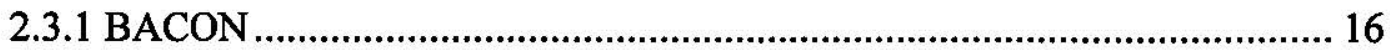

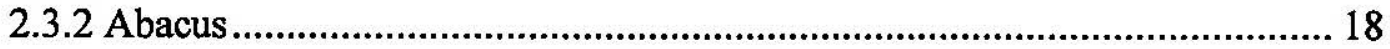

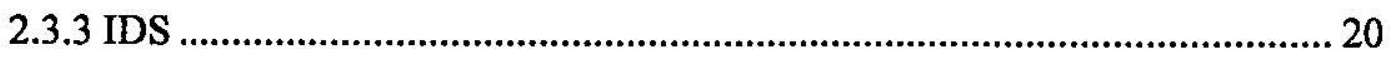

iii 
4.2.1 The Influence of the Number of Variables.................................................67

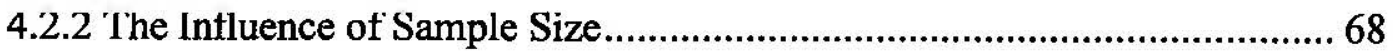

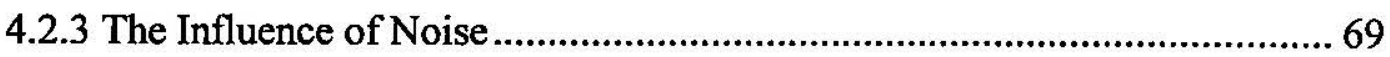

4.3 SOLUTION TO TREE INSTABILITY ........................................................ 70

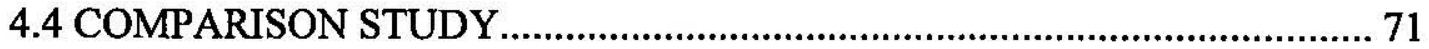

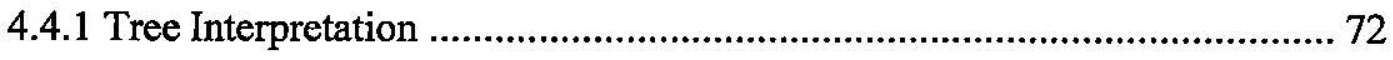

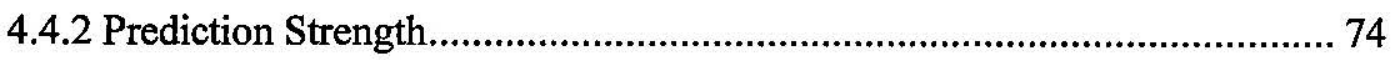

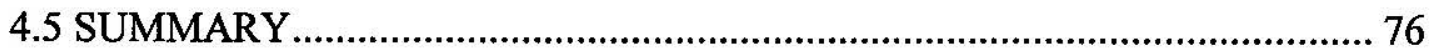

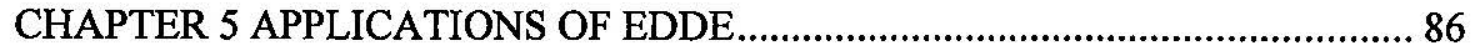

5.1 PLANNING AND SCHEDULING PROJECTS............................................. 86

5.2 DEVELOPMENT/SPLICE STRENGTH OF REINFORCING BARS ............ 88

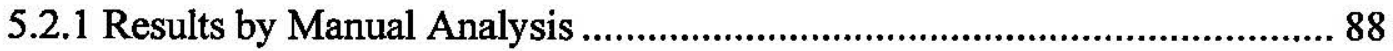

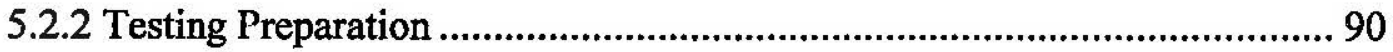

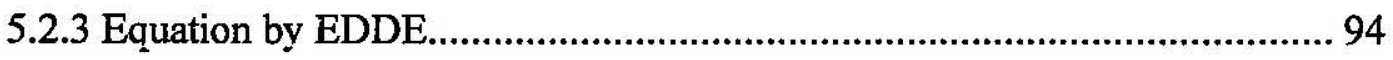

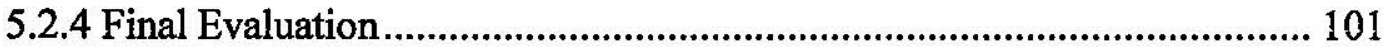

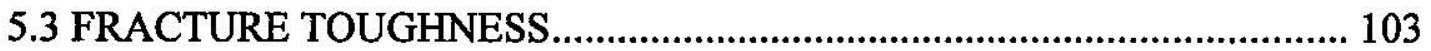

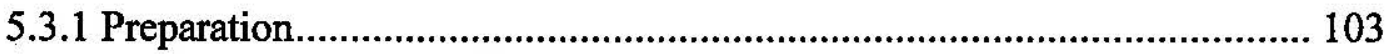

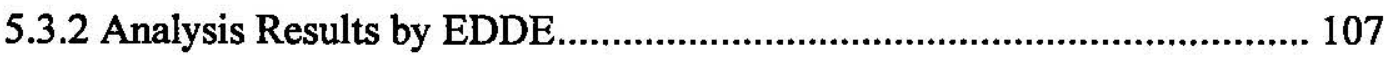

5.4 DISSOLUTION OF IONIZABLE DRUG ...................................................... 110

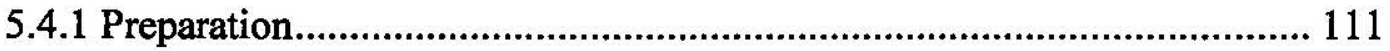

5.4.2 Analysis Results by EDDE.................................................................. 113

5.5 AUTOMOBILE FUEL CONSUMPTION................................................... 115

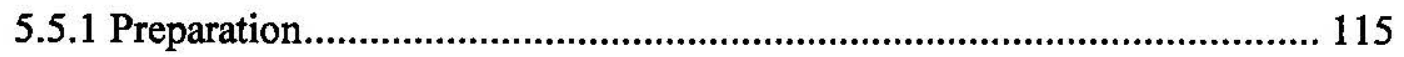




\section{LIST OF TABLES}

Table 1.1 The steps in the KDD process [Fayyard, Piatetsky-Shapiro, Smith, 1996b] 3

Table 2.1 Data obeying Kepler's third law of planetary motion................................ 17

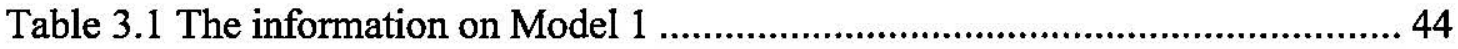

Table 3.2 Variable information on the generated data set.......................................... 44

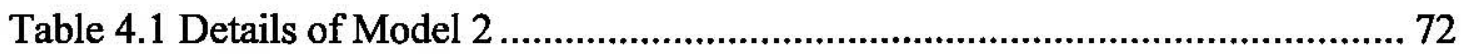

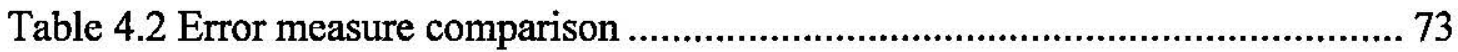

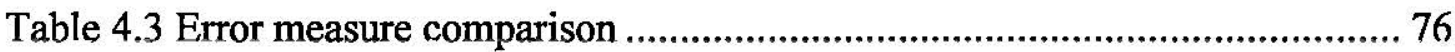

Table 5.1 Discovered equation when the dependent variable is $\mathrm{T}_{\mathcal{d}} / \mathrm{f}_{\mathrm{c}}{ }^{1 / 4} \ldots \ldots \ldots \ldots \ldots \ldots . . .97$

Table 5.2 Results when the dependent variable is $\mathrm{T}_{\mathrm{c}} / \mathrm{f}_{\mathrm{c}}{ }^{\mathrm{p}}(\mathrm{p}=0.22 \sim 0.50) \ldots \ldots \ldots \ldots \ldots \ldots . . .98$

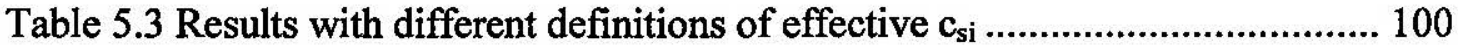

Table 5.4 Evaluation of the induced equation .......................................................... 102

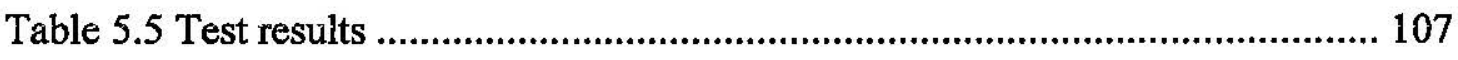

Table 5.6 Model drugs............................................................................................. 111

Table 5.7 Performance of induced equation at different stages............................... 119

Table 5.8 Results by different percentage of testing data ....................................... 120

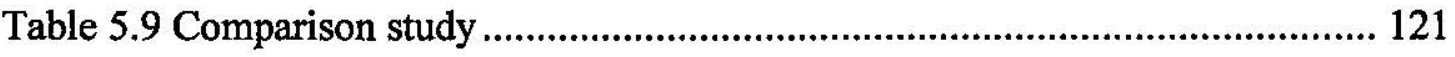


Fig. 5.1 Process of managing a project .......................................................... 127

Fig. 5.2 Test set-up and beam configuration .................................................... 128

Fig. 5.3 Fracture surface at splice failure ……………......................................... 129

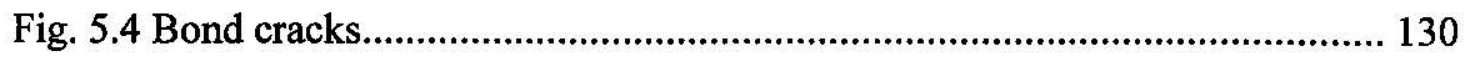

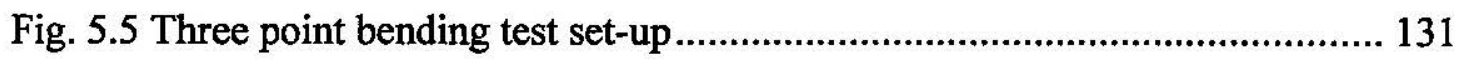

Fig. 5.6 Schematic relationship between CTOD and temperature............................. 132

Fig. 5.7 Schematic effect of loading rate on CTOD-temperature relationship......... 132

Fig. 5.8 Influence of crack depth and a/W ration on $\ln (\mathrm{CTOD}) \ldots \ldots \ldots \ldots \ldots \ldots \ldots \ldots \ldots \ldots \ldots . . . . . . . . . . . . . .133$

Fig. 5.9 Constant crack depth and varying a/W ratio............................................ 134

Fig. 5.10 Constant crack depth and varying a/W ratio......................................... 134

Fig. 5.11 Varying crack depth and constant a/W ratio............................................ 135

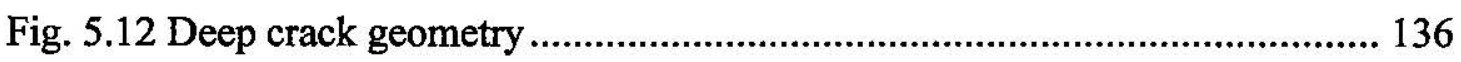

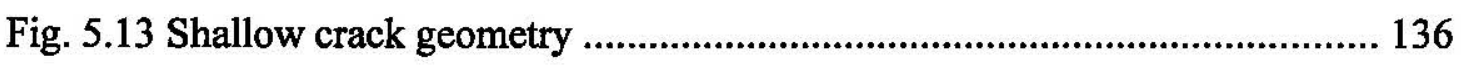

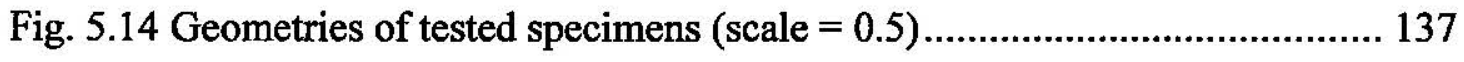

Fig. 5.15 Schematic diagram of the dissolution cell ............................................. 138

Fig. 5.16 Flux prediction when pKs=4.02 ........................................................ 139

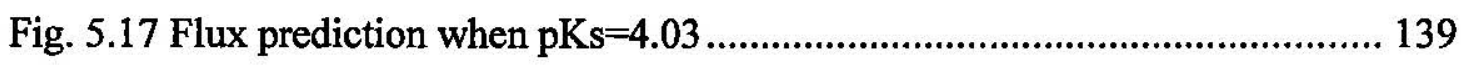

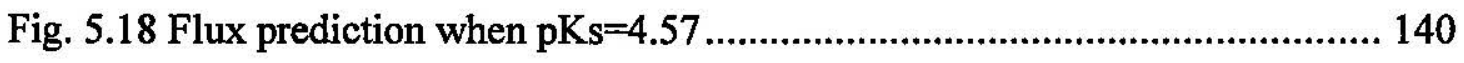

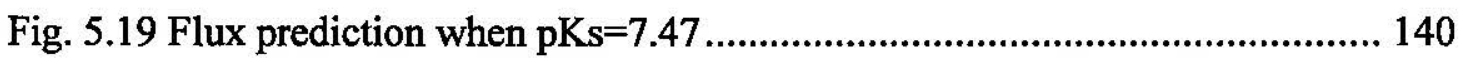

Fig. 5.20 Overall performance of predicting flux ................................................. 141

Fig. 5.21 Prediction performance on fuel consumption ......................................... 142

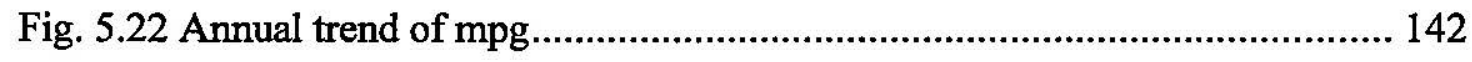




\section{CHAPTER 1}

\section{INTRODUCTION}

Engineering is data rich. The data collected for most engineering purposes are generally associated with sets of observations and examples from problem domains. However, the data sets have limited usefulness because they describe the behavior of the domains only at the level of examples, while providing no insight into the domains.

To better understand engineering domains, engineers are often challenged by the need to analyze large databases. Such data analysis has two aspects. On the one hand, analysis of the data offers the opportunities of acquiring useful knowledge implicitly underlying the observed data and using the newly discovered knowledge in conjunction with the existing knowledge. On the other hand, the quantity of data grows and the number of dimensions increases with the easy collection and storage of data by computers. Consequently, manually analyzing the data from engineering domains is time consuming, or even impossible within any practical length of time. Building computer systems to analyze data automatically and intelligently becomes more and more important for engineers to overcome the difficulties of dealing with large quantities of data to acquire useful knowledge on the domains.

This thesis is concerned with building an intelligent computer system to help engineers in acquiring knowledge. This chapter first gives an overview of research in terms of data mining, machine learning, and intended problem characteristics, then closes with a brief description of the organization of this thesis. 
Table 1.1 The steps in the KDD process [Fayyard, Piatetsky-Shapiro, Smith, 1996b]

1. Learning the application domain: includes relevant prior knowledge and the goals of the application.

2. Creating a target dataset: includes selecting a dataset or focusing on a subset of variables or data samples on which discovery is to be performed.

3. Data cleaning and preprocessing: includes basic operations, such as removing noise or outliers if appropriate, collecting the necessary information to model or account for noise, deciding on strategies for handling missing data fields, and accounting for time sequence information and known changes, as well as deciding DBMS issues, such as data types, schema, and mapping of missing and unknown values.

4. Data reduction and projection: includes finding useful features to represent the data, depending on the goal of the task, and using dimensionality reduction or transformation methods to reduce the effective number of variables under consideration or to find invariant representations for the data.

5. Choosing the function of data mining: including deciding the purpose of the model derived by the data mining algorithm (e.g., summarization, classification, regression, and clustering)

6. Choosing the data mining algorithm(s): includes selecting method(s) to be used for searching for patterns in the data, such as deciding which models and parameters may be appropriate (e.g., models for categorical data are different from models on vectors over reals) and matching a particular data mining method with the overall criteria of the KDD process (e.g., the user may be more interested in understanding the model than in its predictive capabilities)

7. Data mining: includes searching for patterns of interest in a particular representational form or a set of such representations, including classification rules or trees, regression, clustering, sequence modeling, dependency, and line analysis

8. Interpretation: includes interpreting the discovered patterns and possibly returning to any of the previous steps, as well as possible visualization of the extracted patterns, removing redundant or irrelevant patterns, and translating the useful ones into terms understandable by users

9. Using discovered knowledge: includes incorporating this knowledge into the performance system, taking actions based on the knowledge, or simply documenting it and reporting it to interested parties, as well as checking for and resolving potential conflicts with previously believed (or extracted) knowledge 
Mining offers a promising approach to attack the problem.

Based on the consideration of the stated characteristics, the method proposed combines a machine learning technique and regression analysis to automatically and intelligently help in discovery of knowledge hidden in data.

\subsection{MACHINE LEARNING}

Learning from databases with a set of examples or observations is called empirical learning or inductive learning. This type of learning is one of the most extensively studied in machine learning and statistics, and is the subject of this thesis.

Empirical learning systems can compress and abstract a large data set into a higher level compact model that more concisely describes the data. Based on the degree of supervision a system obtains, there are two techniques that use given examples to derive a concept: supervised learning and unsupervised learning.

In the activities of supervised learning, a tutor or domain expert provides the information about which variable to focus on during the learning task. In other words, the domain variables are divided as dependent variables and independent variables for supervised learning, in sharp contrast to unsupervised learning activities where such information is absent. However, many methods designed for supervised learning problems can be adapted to unsupervised ones [Langley, 1996].

For most engineering data analysis tasks, the domain knowledge provides sufficient information for engineers to decide which variables to focus on. This is the reason that this thesis aims at supervised learning instead of unsupervised learning. The learning activity scenario of interest in this thesis is one that happens quite often in engineering, and can be stated as follows:

Given a data set, $L$, with $N$ examples $L=\left\{\mathbf{e}_{1}, \mathbf{e}_{2}, \ldots, \mathbf{e}_{N}\right\}$. Each example $\mathbf{e}_{i}$ in the data set has one targeted dependent variable $y$ and a fixed number (M) of variables $\mathrm{X}=\left\{x_{1}, x_{2}, \ldots, x_{\mathrm{M}}\right\}$ in a domain space $\mathrm{D}$, where $\mathrm{X} \in \mathrm{D}$. Therefore, $\mathrm{e}_{i}$ 
Generally, an engineering database includes high dimensionality, a mixture of data types, and different relationships holding between variables in different parts of the domain space as mentioned in Section 1.1. Furthermore, engineering problems are various. Building a system that can learn from all kinds of engineering databases does not appear feasible with current computational techniques. Instead, this research presents a method suitable for a particular set of characteristics of the database and acquired equations as discussed in the next section.

\subsection{INTENDED PROBLEM CHARACTERISTICS}

Frequently in engineering domains, both prediction and description are equally stressed. The functions found from data require not only satisfactory prediction on unseen or new cases, but also understandability to the domain engineers. Therefore, engineers want to introduce a simple, understandable model based on the domain knowledge in guiding data analysis. That is to say, engineers give their expectations about possible forms of the numerical relationships. The introduction of domain knowledge restricts the relationships that can be found. The domain knowledge of the problem type intended for solution by the proposed method includes:

- The problem space is multi-dimensional and nonhomogeneous. Therefore, Eq. (1.1) can be more explicitly expressed as

$$
\mathrm{R}_{i}: \mathrm{y}=f_{i}\{\mathrm{X}\}
$$

where $\mathrm{R}_{i}$ are regional descriptions and $f_{i}$ are regional equations.

- Independent variables $\mathbf{X}$ are divided into description variables and prediction variables. Description variables are used in region descriptions $\mathbf{R}_{i}$, and prediction variables are used in region equations $f_{i}$. The prediction variables have to be numeric while the description variables can either be numeric or symbolic. However, if a numerical variable is used as 
affect the degree of the influence of the road length. That is to say, the influence of the road length behaves differently when projects are located in different regions. This is reflected in equation expressions where the same relationship does not hold over the entire problem domain so that different equations hold in different parts of the problem space. In the actual situation, the problem is not so simple because many project attributes (or variables as they are called in general data analysis) are involved in the database with historical records of past construction. The road construction projects in the database can be used as a training set of examples which the data mining method uses to build a model to predict activity durations based on project information including length, tracts, location, and situation. Models could also be built to describe the data in a way that lets the user better understand what the data means, for example, by looking at work done by various contractors and seeing what project types were best done by what contractors.

This planning and scheduling case is one example of the type of engineering learning that is the subject of this thesis.

\subsection{THESIS ORGANIZATION}

The remainder of this thesis is organized as follows:

Chapter 2 first introduces the concept of learning. The definition by Langley [1996] is given, which the author believes is the most broad and accurate. Then, the chapter reviews the machine learning methods in five paradigms according to knowledge representation. Finally, the chapter discusses in detail the systems designed for function discovery, which are related to the research presented in this thesis. Their learning capabilities and shortcomings are summarized.

Chapter 3 describes EDDE, a learning system for Equation Discovery in Databases from Engineering. The chapter discusses the technical approach specified for EDDE in terms of the learning task, knowledge representation, equation discovery 


\section{CHAPTER 2}

\section{REVIEW OF RELATED WORK}

Learning is viewed as the central feature of intelligent systems. This chapter starts with a brief preview of the definition of learning. Then, it reviews important categories of machine learning techniques grouped by knowledge representation. Finally, it reviews in more detail the machine learning techniques for numerical law discovery, which are closely related to the research presented in this thesis.

\subsection{PREVIEW}

It would be satisfying to here state a clear, unambiguous definition of learning. Unfortunately, any attempt to draw a fixed boundary around such a broad concept is doomed to failure. Although one can give a precise formal definition, others can always find intuitive examples that fall outside the specified boundaries and counterexamples that fall within them. However, many definitions have been given before and some of them are reviewed in [Reich and Fenves, 1989]. Among the previous definitions, the author believes that the definition given by Lanley [1996] is the broadest and the most accurate:

Learning is the improvement of performance in some environment through the acquisition of knowledge resulting from experience in that environment.

This definition states that learning can only happen with the presence of four 
machine learning techniques are classified differently. Here Langley's classification of machine learning techniques is adopted, which classifies the machine learning techniques into five paradigms according to knowledge representations [Langley, 1996].

\subsubsection{Neural Networks}

Neural networks represent knowledge as a multilayer network of threshold units that spreads activation from input nodes through internal units to output nodes. Weights on the links determine how much activation is passed on in each case. The neural network typically attempts to improve the accuracy of classification or prediction by modifying the weights on the links. A comprehensive presentation of various neural networks is given in [Freeman and Shapura, 1991; Shapura, 1996].

\subsubsection{Instance-Based or Case-B ased Methods}

Instance-based methods, rather than forming some abstract and storing this structure in memory, represent knowledge in terms of specific cases or experiences and rely on flexible matching methods to retrieve these cases and apply them to new situations. Different algorithms are developed based on the matching methods. This paradigm contains methods such as nearest neighbor algorithms [Cover and Hart, 1967; Dasarathy 1991], k-nearest neighbor algorithms [Stanfill and Waltz, 1986], and average-case analysis [Langley and Iba, 1993].

\subsubsection{Genetic Algorithms}

Genetic algorithms were derived from the evolutionary model of learning [Forsyth, 1989]. They typically represent acquired knowledge as a set of Boolean or binary features, which are sometimes used as the conditions and actions of rules. Genetic algorithms use the Darwinian principle of 'survival of the fittest'. A genetic classifier is comprised of a set of classification elements that replicate and mutate to 
As Langley points out, the reasons for the distinct identities of these paradigms are more theoretical than scientific [Langley, 1996]. Recent development in the machine learning community has helped break down these boundaries, and hybrid approaches that cross these boundaries are increasingly common.

\subsection{NUMERICAL LAW DISCOVERY}

Most methods discussed above construct knowledge structures that classify objects into a finite number of classes. We may refer to them as classification systems. For some applications, however, we would like to predict the value, usually numeric, of an attribute of interest. If we still adopt the classification methods, we can construct decision rules or other forms of knowledge, but some problems arise including the following.

If we construct decision rules that predict the unknown value, we would end up with as many rules as these are different values. A solution to this is to discretize these values by mapping them into a finite number of classes. Methods taking this discretization approach include CART [Breiman, Friedman, Olshen and Stone, 1984] and M5 [Quinlan, 1992]. However, discretization involves a loss of information and prediction accuracy will be influenced. The prediction accuracy depends on how finely the classes are discretized.

If we construct other forms of knowledge such as a neural network, the knowledge underlying the data is not explicitly expressed. Such implicit classification methods are unsatisfying for engineering applications that use models for both prediction and description.

As engineers, we would like the knowledge in the domain to be expressed as numerical functions because they clearly and definitely state the relationship among domain variables qualitatively and quantitatively. Therefore, previous work related to the knowledge acquisition process of numerical law discovery is particularly relevant. 
revolution $p$ are related as $\frac{d^{3}}{p^{2}}=k$ where $\mathrm{k}$ is a constant.

Table 2.1 Data obeying Kepler's third law of planetary motion.

\begin{tabular}{|c|c|c|c|c|c|}
\hline Planet & $d$ & $p$ & Term-1: $\frac{d}{p}$ & Term-2 $: \frac{d^{2}}{p}$ & Term-3: $\frac{d^{3}}{p^{2}}$ \\
\hline $\mathrm{A}$ & 1 & 1 & 1.0 & 1.0 & 1.0 \\
\hline $\mathrm{B}$ & 4 & 8 & 0.5 & 2.0 & 1.0 \\
\hline $\mathrm{C}$ & 9 & 27 & 0.33 & 3.0 & 1.0 \\
\hline
\end{tabular}

A set of examples, i.e. the values of $d$ and $p$ for three (strictly hypothetical) planets, are given in the second and third columns of the Table 2.1. Based on the observation that $d$ and $p$ increase monotonically, the term $\frac{d}{p}$ is constructed. This term is not constant, we can see in the fourth column, so term construction continues. Since $d$ and $\frac{d}{p}$ vary inversely, a new term $d\left(\frac{d}{p}\right)=\frac{d^{2}}{p}$ is constructed. This term varies inversely with $\frac{d}{p}$, so their product $\frac{d^{3}}{p^{2}}$ is computed. Computation of this term results in a constant for all examples. The algorithm reports it as the numerical relationship that can describe all the data, and then stops.

BACON has some requirements for input data. It requires enough data to be gathered so it is always possible to observe changing values of two variables while holding the others constant. This requirement can be satisfied if the data comes from controllable experiments. Furthermore, it allows no irrelevant variables in the data provided. That is to say, all variables are relevant. This requirement suggests all variables are present in the function reported by the system, which is quite often not the case in engineering domains. 
among the variables.

Abacus does allow irrelevant variables to be present in the input data. The presence of irrelevant variables in data sets exacerbates the problem that combining existing variables to form new ones is inherently exponential. To limit searching, Abacus uses three constraints that prevent mathematically redundant or physically impossible equations from being formed. First, Abacus prohibits redundant terms. For example, if a term $\frac{x y}{z}$ has been created, this strategy will prevent the term formation of $\frac{x}{z} y$. Second, Abacus prohibits combinations that would result in cancellation. For example, this strategy prevents the discovery of tautologies such as the invariance of $\frac{x y}{x y}$. Third, Abacus applies the notion of dimensional analysis. For example, if two terms $x$ and $y$ are measured in different units, this strategy prevents the formation of new terms of $x+y$ and $x-y$.

Another innovation introduced into Abacus is its ability to handle cases in which different functional relationships govern different parts of the input data set. Abacus deals with multiple relationships by finding formulas which are invariant over a significant subset of the data, characterizing and removing this subset and then, recursively, analyzing the remaining data. When multiple equations are discovered for a given set of data, Abacus has a separate module to generate a condition with each equation.

Abacus, like BACON, requires enough data to be collected so that it could always be able to observe changing values of two variables while holding the others constant. It is tested on artificial data sets similar to those tested by BACON. Its ability to analyze real data is also weak.

Other related system are Abacus.2 [Greene, 1988], Coper [Kokar, 1986a and 1986b], Fahrenheit [Zytkow, 1987] and Fortyniner [Zytkow and Baker, 1991]. 
IDS's search. That is to say, the system selects the function that fits the training data set, not the one that does the best job of predicting a set of unseen data. In this aspect, IDS is also different from the BACON and Abacus type systems discussed above where predictive accuracy is not a concern.

\subsubsection{Kepler}

Kepler, constructed by $\mathrm{Wu}$ and Wang [1991], is a system designed for discovery of functional relationships from observational data. As its authors claimed, it is designed to discover more complicated functions than BACON and Abacus. Like BACON and Abacus, Kepler finds functional relationships by detecting the invariant. However, the relationship is not expressed as a term but as

$$
f\left(x_{1}, x_{2}, \ldots x_{n}\right)=k=\text { constant }
$$

To simplify the problem, Kepler has three assumptions: (1) each variable appears in the formula just once, (2) each operator takes at most two variables as its arguments, and (3) two functions that differ only by a constant (for example, $x^{2}+3$ and $\left.x^{2}+7\right)$ are considered equivalent.

Unlike BACON and Abacus, which form new terms only using the basic arithmetic operations: addition, subtraction, multiplication and division, Kepler uses primitive functions that are defined as a nondivisible part of a formula. Dividing a formula means putting the formula into different parts, with each variable appearing in only one part. A formula can be discovered by discovering its part. This discovery is possible because the parts are independent of each other. Therefore, discovering a complex multivariable formula is accomplished by finding its primitive functions. Some examples of prototypes of primitive functions are

$$
\begin{aligned}
& x^{k_{1}} \times y^{k_{2}} \\
& x^{k_{1}} / y^{k_{2}}
\end{aligned}
$$




\subsubsection{KEDS}

The systems reviewed above are intended to be domain independent while KEDS (Knowledge based Equation Discovery System), as its name implies, is intended to be domain dependent [Rao and $\mathrm{Lu}, 1993$; Rao, 1993]. It attempts to discovery comprehensible models in an engineering domain that is multidimensional and nonhomogeneous. These models are expressed as region-equation pairs of the form:

$$
R_{i}: y=f_{i}\left(x_{1}, x_{2} \ldots\right)
$$

where $R_{i}$ is the description of the region $i, y$ is the predicted (dependent) variable, and $x_{1}, x_{2}, \ldots x_{\mathrm{n}}$ are independent variables. $f_{i}$ is the region related equation, which is a user defined template. The templates represent the domain knowledge. In KEDS, the templates are expressed as families of parameterized models (polynomial equation templates) such as

$$
\begin{aligned}
& y=c \\
& y=a x_{1}+b \\
& y=a x_{1}{ }^{2}+b x_{1}+c \\
& y=a x_{1}+b x_{2}+c
\end{aligned}
$$

where $a, b$, and $c$ are unknown coefficients.

To accomplish the discovery process, KEDS adopts the method of empirical discovery and conccptual clustering. Its algorithm involves two phases: discovery and partitioning. In the discovery phase, the discovery process is restricted within the boundaries of the regions created by the partitioning. In the partitioning phase, partitioning is model-driven and based on the relationships that are discovered from the data. The discovery and partitioning phases are coupled and are thus done interactively, not sequentially. 


$$
\begin{aligned}
& y=k_{1} x+k_{2} \\
& y=k_{1} x^{-2} \\
& y=k_{1} x^{-1} \\
& y=k_{1} x^{-1 / 2} \\
& y=k_{1} x^{1 / 2} \\
& y=k_{1} x \\
& y=k_{1} x^{2}
\end{aligned}
$$

or the null relation equivalent to "none of the above". Three measures, significance, distinction, and systematic lack of fit, are used to do the classification. The $\mathrm{E}^{*}$ algorithm focuses, in fact, on improving the reliability of direct curve finding but not on function finding [Schaffer, 1991]. For this reason, the $\mathrm{E}^{*}$ algorithm is not discussed further here. More details can be found in [Schaffer, 1990]. 
equation discovery in engineering problems. On the one hand, different engineering problems have their own characteristics and need different methods to solve them; on the other hand, a specific method has its limitations and is not applicable to all kinds of problems.

EDDE is designed for a coherent class of problems with the characteristics and goals discussed in Chapter 1, which attempts to find a relationship between a dependent variable and other independent variables, expressed as region-equation pairs shown in Eq. (1.2). The task is restated here:

Given a data set, $L$, with $N$ examples $L=\left\{\mathbf{e}_{1}, \mathbf{e}_{2}, \ldots, \mathbf{e}_{N}\right\}$. Each example $\mathbf{e}_{i}$ in the data set has one targeted dependent numerical variable, $y$, and a fixed number $\mathrm{M}$ of variables $\mathbf{X}=\left\{x_{1}, x_{2}, \ldots, x_{M}\right\}$ in a domain space $\mathbf{D}$, where $\mathbf{X} \in$ D. Therefore, $\mathbf{e}_{i}=\{\mathbf{y}, \mathbf{X}\}$. The data analysis requires finding a model between the dependent variable $y$ and the independent variables $\mathbf{X}$, which is expressed as

$$
\mathbf{R}_{i}: y=f_{i}\{\mathbf{X}\}
$$

where $\mathrm{R}_{i}$ is the region description that sets region boundaries; and $f_{i}$ is the equation related with region $i$. The region descriptions are determined by description variables, and the region equations are determined by prediction variables. The information about description variables and prediction variables is provided by domain engineers based on the domain knowledge before the learning begins.

EDDE is targeted to apply to real world engineering problems. Therefore, the given data is almost guaranteed to be noisy with not all of the data points satisfying the model. The only solution is to learn an approximate model. Hence, Eq. (3.1) becomes

$$
\mathrm{R}_{i}: y=f_{i}\{\mathbf{X}\}+\mathrm{Z}\left(0, \sigma^{2}\right)
$$

where the term $\mathrm{Z}$ is added to reflect the noise existing in the data. It is assumed that $\mathrm{Z}$ 
learning partial models and then combining the partial models into a complete model as KEDS does [Rao, 1993]. A partial model is referred to as one region-equation pair in KEDS.

Learning a model represented as region-equation pairs involves learning both region descriptions and region equations. The regional equation $f_{i}$ may be any function of the prediction variables. It may be a linear or nonlinear function. When only linear functions are taken into account, $f_{i}$ may be expressed as Eq. (1.3). Note that this equation gives only the general form, not the actual function, because significant variables in the equation are not identified until the model is induced.

In many engineering domains, linear functions can not give satisfactory results. Nonlinear functions are necessary for engineering problems. When the linear function restriction is relaxed, nonlinear functions may appear in regression models. Based on the characteristics appearing in regression models, nonlinear functions can be divided into two types: intrinsically linear and intrinsically nonlinear [Draper and Smith, 1981]. These two types of nonlinear functions are explained by Draper and Smith as follows:

Two examples of such models are

$$
\begin{aligned}
& Y=\exp \left(\theta_{1}+\theta_{2} t^{2}+\varepsilon\right) \\
& Y=\frac{\theta_{1}}{\theta_{1}-\theta_{2}}\left(e^{-\theta_{2} t}-e^{-\theta_{1} t}\right)+\varepsilon
\end{aligned}
$$

In these examples the parameters to be estimated are denoted by $\theta$ 's

$t$ is the single independent variable, and $\varepsilon$ is a random error term with $\mathrm{E}(\varepsilon)=0, \mathrm{~V}(\varepsilon)=\sigma^{2}$

The models (a) and (b) are both nonlinear in the sense that they involve $\theta_{1}$ and $\theta_{2}$ in a nonlinear way but they are of essentially different character. The model (a) can be transformed, by taking 
Nonlinear functions are introduced in the form of function templates. The nonlinear function templates are expressed in the following form

$$
y \propto g_{i}(\mathbf{X})
$$

where $y$ is the target or dependent variable, and the $g_{i}$ are nonlinear function templates. The sign " $\propto$ " indicates that the targeted variable $y$ should be qualitatively proportional to the nonlinear function of $g_{i}(X)$ if the template is truly in the final induced model. The followings are some template samples:

$$
\begin{aligned}
& y \propto x_{1}^{2} \\
& y \propto \sin \left(x_{1}\right) \\
& y \propto x_{1} \times x_{2} \\
& y \propto e^{x_{1}}
\end{aligned}
$$

The templates can be any nonlinear functions as long as they are based on and supported by the already existing domain knowledge. However, the system decides, according to the data, whether or not a template appears in the induced model. Therefore, when nonlinearity is considered, the region-equation pairs are expressed as a parameterized model and Eq. (3.2) becomes

$$
R_{i}: y=c_{0 i}+c_{1 i} g_{1}(\mathbf{X})+c_{2 i} g_{2}(\mathbf{X})+\ldots+c_{m i} g_{m}(\mathbf{X})+Z
$$

where $g_{1}(\mathbf{X}), g_{2}(\mathbf{X}), \ldots, \mathrm{g}_{m}(\mathbf{X})$ are user defined nonlinear function templates that are understandable to the domain engineers and consistent with the existing domain knowledge. To be more general, $g_{(}(X)$ can be any function template, including linear and nonlinear function templates.

\subsubsection{Equation Discovery as Search}

To find equations expressed as region-equation pairs, both region description and region equation must be learned. The central problem for this learning is to 
somewhere between the top and the bottom of the hierarchy, which is applicable to the kind of learning tasks with the characteristics discussed in Chapter 1.

The nature of the heuristic search process is determined by four basic issues [Langley, 1994; Blum and Langley, 1997].

The first issue is the starting point in the space, which in turn affects the direction of the search. Two extreme approaches are forward selection and backward elimination. Forward selection starts with no feature and successively adds significant variables, whereas backward elimination starts with all variables and successively removes insignificant variables. Some approaches may be somewhere between the two and start with some features at the beginning. For the intended task, the starting points of searching the variable spaces are different for the variable identifications used in region descriptions and region equations. Forward selection is used to identify the significant variables in region descriptions, and backward elimination is applied to identify the significant variables in region equations.

The second issue is the organization of the search. As seen at the beginning of this section, exhaustive search is not practical for most real world engineering problems. A greedy search method is adopted for the intended learning task, which is a method that considers all possible ways within the allowed constraints at each step, evaluates them in terms of an evaluation function in the step, and then selects the best one for the step.

The third issue concerns the strategy used to evaluate alternative variables and select the best variable. Some induction algorithms incorporate a criterion based on information theory as in C4.5 [Quilan, 1994] for their specific targeted learning task. Others measure the accuracy on the training data set or a separate test data set. A broader issue concerns how the feature selection strategy interacts with the basic induction algorithm. EDDE selects error reduction as the strategy to evaluate the alternative variables as discussed in Section 3.2. 
function that fits the training data set, not the one that does the best job of predicting a set of unseen data.

KEDS attempts to discover comprehensible models in an engineering domain. These models are multidimensional and nonhomogeneous. KEDS evaluates generated models along two dimensions: human comprehensibility and accuracy in predicting unknown values.

Like KEDS, EDDE is a system designed to be applied to actual engineering domains. Therefore its evaluation should also be based on both human comprehensibility and predictive strength on the cases that were not accessed to build the model.

Comprehensibility is inversely related to the complexity of a model. The complexity of the model expressed as region-equation pairs depends on the complexity of region descriptions as well as the complexity of associated equations. In general, for a model to have low complexity, it should have as few regions as possible. To further reduce complexity, the equations associated with regions should have as few variables as possible. Therefore, model complexity is measured using two dimensions: model size and leaf size. Model size $(\mathrm{ms})$ is defined and expressed as

$$
\begin{aligned}
m s & =\text { the number of leaves in a model tree } \\
& =\text { the number of regions in a final model }
\end{aligned}
$$

and leaf size $(l s)$ is defined and expressed as

$$
l s=\frac{N_{V}}{N_{R}}
$$

where $N_{V}$ is the total number of variables in all regions and $N_{R}$ is the total number of regions.

Model size is the primary factor and leaf size is the secondary factor that determines the model complexity. Leaf size should be considered only after the 
with higher comprehensibility will be preferable. The parameter $\varepsilon$ defines the percentage of predictive error that can be sacrificed to get higher comprehensibility of region equation pairs. In essence, $\varepsilon$ is a parameter that measures the trade-off between model complexity and model prediction error.

There is a system design choice as to whether the region-equation pairs are reported to users in all circumstances. BACON and the previously discussed systems like it will not report any equation if the preset criterion is not satisfied. However, EDDE always reports the region-equation pairs in each batch of the learning process. It is believed that, based on the feedback information given by EDDE while reporting the region-equation pairs, users have several choices, including using the reported region-equation pairs as equations adequate to describe the data for the intended purpose, modifying the introduced nonlinear function templates, and adjusting model formation parameters (discussed in Section 3.3.3) to start the learning process again. To obtain useful and satisfactory model discovery, domain engineers must cooperate by not only supplying the required data and available domain knowledge but also paying attention to the findings made by EDDE. Any useful discoveries are likely to occur only through interaction between domain engineers and the system. Chapter 5 illustrates the importance of the interaction between domain engineers and the system.

\subsection{EDDE SYSTEM}

Based on the consideration of the technical aspects discussed in Section 3.1, EDDE is built up to accomplish the intended learning task: equation discovery in databases form engineering. The algorithm used to find the region-equation pairs capable of describing the data is discussed in this section. 
with $\mathrm{T}_{i}$ examples. (For comparison, CART chooses a test to give the greatest expected reduction in either variance or absolute deviation). The algorithm uses a greedy search through the non-ancestor prediction variables one by one to choose the prediction variable that maximizes the expected error reduction. This process is repeated on the subsets until every subset either contains few cases, or no other prediction variable is left for further splitting, or the error measure is less than a user defined threshold $\Delta . \Delta$ is one of the control parameters for model induction discussed in Section 3.3.3.

Multivariate models (in the form of general linear models) are constructed for the cases at each node of the model tree, using standard regression analysis [Press, Teukolsky, Vetterling and Flannery, 1988]. However, instead of using all templates (linear and nonlinear) in the standard regression analysis of each node, the templates used in a node are restricted to the templates inherited from its parent node.

After each multivariate model is obtained as above, it is simplified by eliminating templates to minimize its weighted standard deviation after the node variable is identified. Weighted standard deviation ( $w s d$ ) of a node is defined as

$$
w s d=\sum \frac{\mathrm{T}_{i}}{\mathrm{~T}} s d\left(\mathrm{~T}_{i}\right)
$$

The templates are removed one by one through greedy search until no more templates reduce the weighted standard deviation. In the multivariate model the remaining templates at a node will be given to its child nodes.

The multivariate model is further simplified by introducing another model formation parameter $\delta$. For the same data set with noise, the standard deviation will generally decrease with the introduction of more prediction variables. However, the variables that are independent of each other and have no influence on the dependent variable will not result in much decrease in the standard deviation if the variables are added to the equation. These variables should be removed. But, if only the values of standard deviation are taken into account, they will not be removed, even though 
The prediction error will decrease or remain essentially the same as a tree is pruned upward, and then will increase rapidly as the tree is overpruned.

For each examined node, the algorithm chooses as the final model for this node either the simplified multivariate model in the node or the model subtree, depending on the prediction error comparison between the simplified multivariate model and the model subtree. If the multivariate model is chosen, the subtree at this node is pruned and the node becomes a leaf.

In the pruning process, if the prediction error of a node is less than that of its subtree, the node is pruned to a leaf. If the prediction errors of a node and its subtree are comparable, the consideration of the trade-off between comprehensibility and predictive strength will determine whether or not the node should be pruned. The prediction errors of a node and its subtree are said to be comparable if

$$
\left|\frac{e_{n}-e_{s}}{e_{n}}\right|<\varepsilon
$$

where $e_{\mathrm{n}}$ and $e_{\mathrm{s}}$ are the prediction errors of the node and its subtree. $\varepsilon$ is the same as in Eq. (3.10) and will be discussed Section 3.3.3.

\subsubsection{EDDE Algorithm}

Given a data set and templates based on the domain knowledge, EDDE will induce region equation pairs based on the following algorithm.

1. Divide the data into training and testing sets

2. Initialize the tree:

3. Grow a tree starting at the root while there is a generation \{

while there is a node at the current generation \{

$$
\text { if }(s d>\Delta \text { or }
$$


depends on many factors, such as data set size, and computer speed. On relatively small data sets, such as those discussed in Chapter 5, each batch of learning (Repeat set to default value of 20) can be finished within a minute on Pentium II with a clock speed of 350 . For larger size data sets, such as a data set with 50 variables and 800 examples, each batch of learning can be finished within 20 minute on Pentium II with a clock speed of 350 .

EDDE learns a complete model from data in a batch scenario. At the beginning of the learning process, users may specify, in addition to the introduction of nonlinear function templates, the model formation parameters for EDDE. In the end, EDDE reports what is found and provides feedback information about the discovery to the users. The users then decide whether to terminate the learning, or to modify the model formation parameters or templates for continuing the learning process. This section discusses the implementation of the algorithm and shows how a model tree is built through an example.

\subsubsection{Model Induction}

The algorithm of EDDE is presented in Section 3.2.3 and its detailed implementation is shown through the following example. The data set used in this example has 200 data points. It is generated based on Model 1 with $\sigma^{2}=10$ by MATLAB [MATLB, 1992]. The details of Model 1 are given in Table 3.1 and the details of the generated data are given in Table 3.2. The complete data set is listed in Appendix A.

With the given data and the default model formation parameters $t=30, \Delta=0$, $\delta=10$, and $\varepsilon=10$, EDDE reports region-equation pairs that correspond to the induced tree shown in Fig. 3.2. The figure shows that the known function and the induced equations are the same except for small differences in the corresponding coefficients. Now we will show how EDDE induces the equations. 


$$
\begin{aligned}
s d(\mathrm{~T}) & =s d\left(\mathrm{~T} ; x_{6}, x_{7}, x_{8}, x_{9} \text { and } x_{10}\right) \\
& =\sqrt{\frac{\sum_{i=1}^{N}\left(y_{i}-\hat{y}_{i}\right)}{N-M-1}}=\sqrt{\frac{\sum_{i=1}^{140}\left(y_{i}-\hat{y}_{i}\right)}{140-5-1}}=29.151
\end{aligned}
$$

where $\mathrm{N}$ is the number of the examples in the node, $\mathrm{M}$ is the number of the variables $\left(x_{6}, x_{7}, x_{8}, x_{9}\right.$, and $\left.x_{10}\right)$ in the node, $\hat{y}_{i}$ is the predicted value of the $i$ th example by the equation that includes $x_{6}, x_{7}, x_{8}, x_{9}$, and $x_{10}$. The root node needs to grow because the standard deviation (29.151) is larger than the threshold $\Delta$ which is set at the default value of zero. The selection of a variable used in the root node is done by greedy search as follows. EDDE calculates the error reductions of all possible splits by description variables and selects the split that gives maximum error reduction. The error reductions are

$$
\begin{aligned}
\Delta \text { error by selecting } x_{1} & =s d(\mathbf{T})-\frac{T_{1}}{T} s d\left(\mathbf{T}_{1}\right)-\frac{T_{2}}{T} s d\left(\mathbf{T}_{2}\right) \\
& =29.151-\frac{74}{151} \times 18.694-\frac{77}{151} \times 3.226 \\
& =18.344
\end{aligned}
$$$$
\Delta \text { error by selecting } x_{2}=3.569
$$$$
\Delta \text { error by selecting } x_{3}=-0.582
$$$$
\Delta \text { error by selecting } x_{4}=0.863
$$$$
\Delta \text { error by selecting } x_{5}=-0.325
$$

where $T_{1}$ is one subset of $\mathrm{T}$ with $x_{1}=Y, T_{2}$ is another subset of $\mathrm{T}$ with $x_{1}=\mathrm{N}$, and $\mathrm{T}_{1}+\mathrm{T}_{2}=\mathrm{T}$. $S d$ 's are taken with regard to $x_{6}, x_{7}, x_{8}, x_{9}$, and $x_{10}$. The variable $x_{1}$ gives the maximum error reduction, thus the variable $x_{1}$ is used in the root node. The data will be split according to the values of the variable $x_{1}$ into two branches $\left\langle x_{1}=\mathrm{Y}\right\rangle$ and $<x_{1}=\mathrm{N}>$ (See Fig. 3.3 branches from root node.) 
elimination of $x_{9}$ is smaller, thus $x_{9}$ is eliminated. This process repeats until no other variables should be removed. For this node, no other variables should be further eliminated from the prediction variables. Therefore, only the prediction variables of $x_{6}, x_{7}, x_{8}$ and $x_{10}$ will be inherited and used in its child nodes.

Then, the tree will grow to the second generation. For the branch of $\left\langle x_{1}=Y\right\rangle$, the first node of the second generation, its standard deviation is 18.644 , larger than the threshold $\Delta$, and this node needs to grow. EDDE again calculates the error reduction of all remaining description variables $\left(x_{2}, x_{3}, x_{4}\right.$ and $\left.x_{5}\right)$ and finds the split based on the variable $x_{2}$ will give the maximum error reduction. Thus, the variable $x_{2}$ is selected to be used in the node. Then, EDDE calculates the weighted standard deviation and finds $x_{10}$ should be eliminated. Therefore, only $x_{6}, x_{7}$ and $x_{8}$ will be inherited by its child nodes down below.

For the branch of $\left\langle x_{1}=\mathrm{N}\right\rangle$, the second node of the second generation, its standard deviation is 3.210 , larger than the threshold $\Delta$, and this node also needs to grow. EDDE again calculates the error reduction of all remaining description variables $\left(x_{2}, x_{3}, x_{4}\right.$ and $\left.x_{5}\right)$ and finds the split based on the variable $x_{4}$ will give the maximum error reduction. Thus, the variable $x_{4}$ is selected to be used in the node. Then EDDE calculates the weighted standard deviation and finds $x_{6}, x_{7}$ and $x_{10}$ should be eliminated. Therefore, only $x_{8}$ will be inherited by its child nodes down below.

After EDDE checks all nodes of the second generation, EDDE checks the third generation and so on until the tree stops growing. The tree grows like that in Fig. 3.3 and stops growing because there are not enough examples in the nodes for further splitting thus the nodes become leaves. At this point, the process of growing the tree has been completed.

Before starting its pruning process, EDDE will further simplify the multivariate model in all nodes if the prediction variables do not influence the standard deviation in the nodes significantly (within the threshold $\delta$ ). For example, at the node $\left\langle x_{1}=\mathrm{Y}>\right.$, the first node of the second generation, the standard deviation with 
leaf, which contains the following equation

$$
y=2.213+8.546 x_{8}
$$

Consider another non-leaf node $\left\langle x_{1}=\mathrm{N}>\right.$. The prediction error given by the non-leaf node and its subtree on the testing data are 2.228 and 2.113 respectively. Although the prediction error given by the non-leaf node is larger than that by its subtree, the improvement by keeping the subtree is small and is within the range of $\varepsilon$ $\left(\left|\frac{2.113-2.228}{2.113}\right|=5.44 \%<10 \%=\varepsilon\right)$. The subtree of the node $<x_{1}=\mathrm{N}>$ should be pruned. This pruning process goes on at each non-leaf node of the model tree and the final tree after the pruning is shown in Fig. 3.2. The equations corresponding to the final tree and the information about the induced model are

$$
\begin{aligned}
& \text { if } x_{1}=\mathrm{Y} \text { and } x_{2}=\mathrm{T}, y=4.489+7.949 x_{6}+9.889 x_{7}<s d=2.721, \mathrm{COD}=0.991> \\
& \text { if } x_{1}=\mathrm{N}, \quad y=4.492+7.979 x_{8} \quad<s d=3.195, \mathrm{COD}=0.983> \\
& \text { Model complexity }(\mathrm{ms}) \quad=3 \\
& (l s) \quad=1.667 \\
& \text { Prediction error } \quad=2.547 \\
& \text { Prediction percentage error } \quad=28.955 \% \\
& \text { Prediction accuracy } \quad=91.837 \% \\
& \text { 1-RE } \quad=0.993 \\
& \text { Ratio mean } \quad=0.992 \\
& \text { Variance of ratio mean } \quad=1.218
\end{aligned}
$$$$
\text { if } x_{1}=\mathrm{Y} \text { and } x_{2}=\mathrm{F}, y=7.746+3.927 x_{6}+6.771 x_{7}<s d=2.881, \mathrm{COD}=0.987>
$$

Based on the given information, the user can make the best decision on the induced model. In the following, a description of the feedback information provided by EDDE is given. 
Coefficient of determination (COD) [Neter and el. at, 1996] describes the degree of fit between the dependent variable $y$ and the independent variables in the equation. Coefficient of determination indicates the proportionate reduction in the total variation of $y$ associated with the use of the independent variables in each region. Thus, the larger the $\mathrm{COD}$, the more the total variation of $y$ is reduced by introducing the independent variables in the region. The range of $\operatorname{COD}$ is $[0,1]$. It should be noted that COD is calculated based on training data and is a resubstitution estimate.

The square root of COD

$$
C O R= \pm \sqrt{C O D}
$$

is called the coefficient of correlation (COR) and is another often used measure. However, the coefficient of correlation is not directly given because it does not have a clear-cut operational interpretation as coefficient of determination does.

\subsubsection{Model Complexity and Prediction Error}

Model complexity and prediction error are the two measures of an induced model as a whole, which are the direct goals the system tries to reach.

Model complexity has two dimensions: model size $(m s)$ defined as the number of the regions of the induced model (Eq. (3.7)) and leaf size $(l s)$ defined as the average number of variables a region (Eq. (3:8)). Model size should be considered as the primary dimension and leaf size should be considered as the secondary dimension. The complexity and the comprehensibility of the model are inversely related. Therefore, the smaller the model size and leaf size, the less complex and the more comprehensible the equations.

Prediction error is defined as the average error given by an induced model on testing data. It is used to measure the prediction strength of a model. The prediction error and the prediction strength are inversely related. Therefore, the smaller the 
where $\hat{y}_{i}$ is the model predicted value of $y$ and $\bar{y}$ is the mean of $y$ in testing data. It is clear that $s_{1}$ represents the mean squared error about the induced model and $s_{2}$ is the mean squared error about the mean of $y$. The relative error (RE) is defined as

$$
\mathrm{RE}=\frac{s_{1}}{s_{2}}
$$

Obviously, (1-RE) gives an estimate of percentage of variation of $y$ explained by the introduction of the induced model. The larger the (1-RE), the better the induced model.

\subsubsection{Ratio Mean and Its Coef ficient of Variation}

Ratio mean $r$ is defined as

$$
r=\frac{1}{\widetilde{T}} \sum_{i=1}^{\tilde{T}} r_{i}=\frac{1}{\widetilde{T}} \sum_{i=1}^{\tilde{T}}\left|\frac{\hat{y}_{i}}{y_{i}}\right|
$$

where $\hat{y}_{i}$ is the predicted value of the $i$ th example by the equation. The coefficient of variation is defined as $r^{\prime}$ s standard deviation as a percentage of its mean [Hogg and Ledolter, 1987]

$$
\operatorname{COV}=\frac{\sqrt{\frac{1}{\widetilde{T}-1} \sum_{i=1}^{\tilde{T}}\left(r_{i}-r\right)^{2}}}{r}
$$

where $\widetilde{T}$ is the number of testing data. Ratio mean and coefficient of variation are also used by some engineers to evaluate a model. Obviously, the closer to 1 the $r$ and the smaller COV, the better the prediction.

\subsubsection{Model Formation Param eters}

There are four model formation parameters that control the learning process. They are test percentage $t$, standard deviation threshold $\Delta$, leaf size trade-off $\delta$ and 
on unseen cases with unnecessary complexity of the tree structure. The influence of the threshold $\Delta$ on the final induced models can be shown using the example below.

Using the same data set in Section 3.3.1, different thresholds $(5,10,20$ and 30) are set while other control parameters remain the same. The induced models under different threshold $\Delta$ are different. Their model complexity and their prediction error are shown in Fig. 3.5 and Fig. 3.6.

It is clear that if the threshold is set lower than the actual standard deviation, the overgrown tree can be pruned to the right size tree so that the overfitting can be avoided and the final induced tree is corresponding to the known model used to generate the data. Low standard deviation threshold takes the advantage of the pruning process so that the model can be discovered. But high standard deviation threshold stops tree growth prematurely, and pruning cannot correct this.

To discover real regularities in data, the solution to the problem is to let the tree grow larger than the right size tree and prune it back. However, in most realworld engineering data, the standard deviation of noise may not known. In these cases, it is hard for users to make the decision how low the threshold should be set so that a tree will not stop growing too early. Therefore, the default value of the standard deviation threshold $\Delta$ is set to zero. By doing so, the tree will not stop growing too early as long as enough data is provided. However users have easy access to change this threshold.

\subsubsection{Trade-off Parameters: $\delta$ and $\varepsilon$}

There are two trade-off parameters. One is the leaf size trade-off parameter $\delta$. The other is the model size trade-off parameter $\varepsilon$. Compared with the formation parameters discussed above, the trade-off parameters have a more critical influence on the final induced tree and its corresponding model region-equation pairs.

The leaf size trade-off parameter $\delta$ is the maximum percentage of 
However, there are some differences between KEDS and EDDE in the use of function templates.

Although the functional relationship is expressed as region-equation pairs both in KEDS and EDDE, a region equation is one of the templates in KEDS but it may be any possible combination of the introduced templates in EDDE.

- The templates in KEDS are expressed as families of parameterized models (polynomial equation templates), such as those in Eq. (2.5), but the templates in EDDE can be any user defined nonlinear functions based on the available domain knowledge. Some sample templates are shown in Eq. (3.5).

- Region-equation pairs in EDDE are expressed using the tree based model with disjoint regions. In KEDS, the induced region-equation pairs may have overlapping regions with decisions as to which equation to use depending on the ordering of region-equation pairs. From the descriptive point of view, it is confusing that a region may be described by more than one equation. The EDDE approach is thus more descriptively clear.

- The organization of search methods is different. KEDS adopts exhaustive search while EDDE adopts greedy search. Therefore, KEDS has a very low learning speed even on small data sets.

\subsection{USE OF DOMAIN KNO WLEDGE IN EDDE}

Frequently in engineering domains, data analysis stresses both prediction and description equally. The function found from data requires not only satisfactory prediction on unseen or new cases, but also understandability to the domain engineers. Therefore, engineers want to introduce domain knowledge in guiding data analysis (Section 1.3). The domain knowledge of the problem type intended for solution by EDDE is discussed in Section 1.3. 


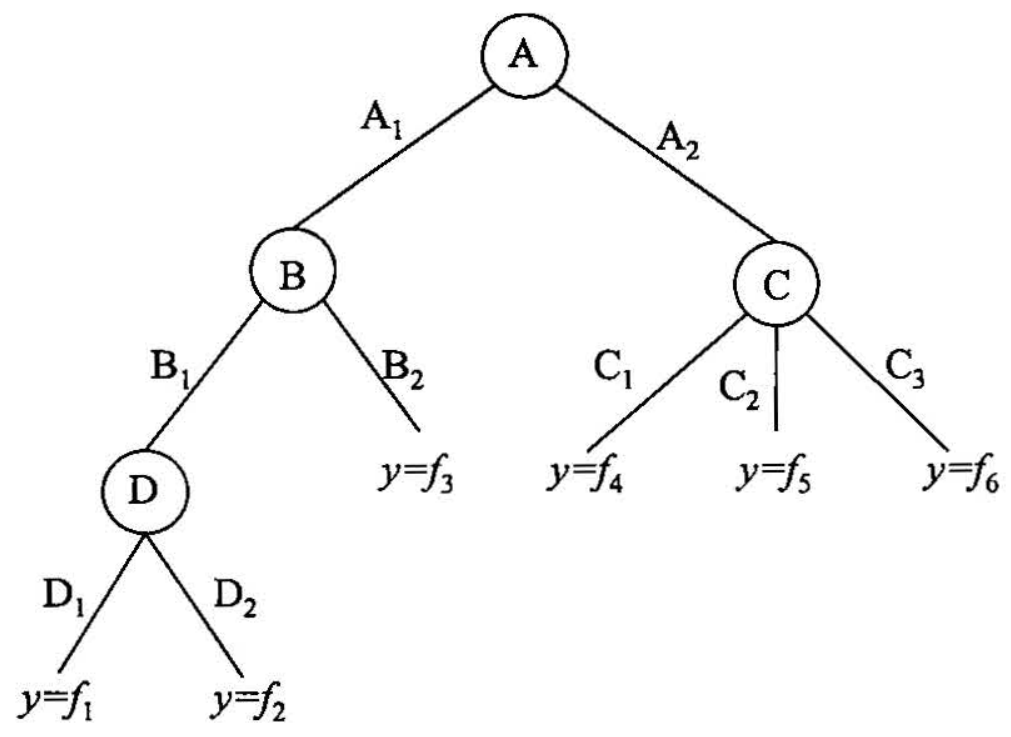

Fig. 3.1 Tree representation of region-equation pairs of Eq.(3.3).

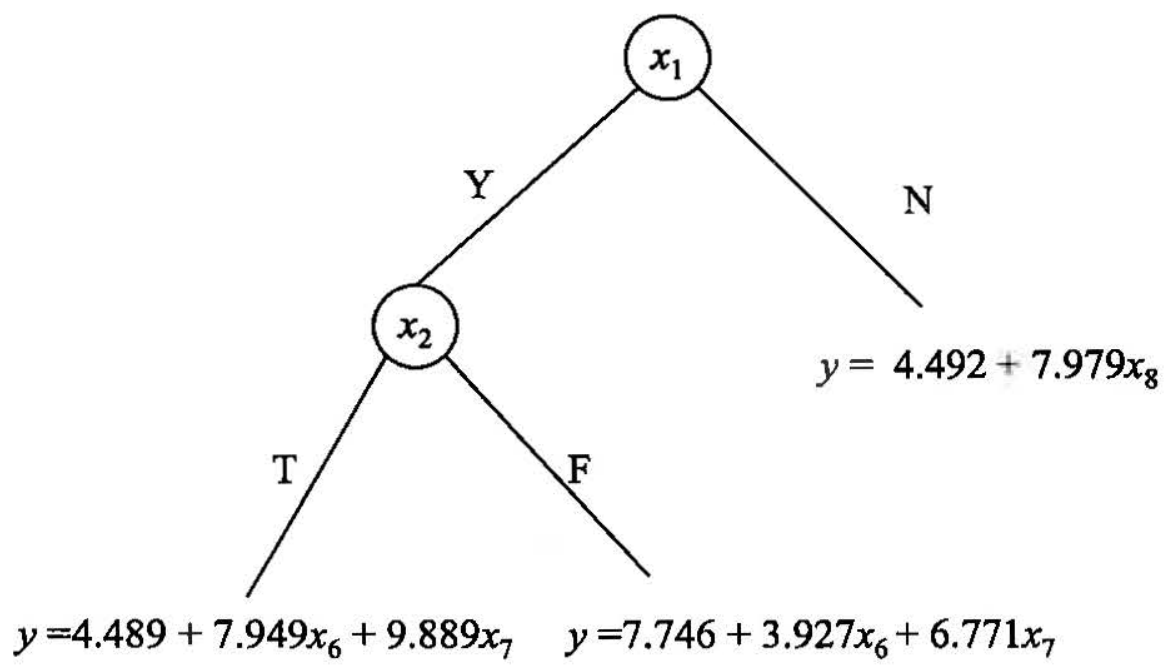

Fig. 3.2 Induced model tree for the example in Section 3.3.1. 


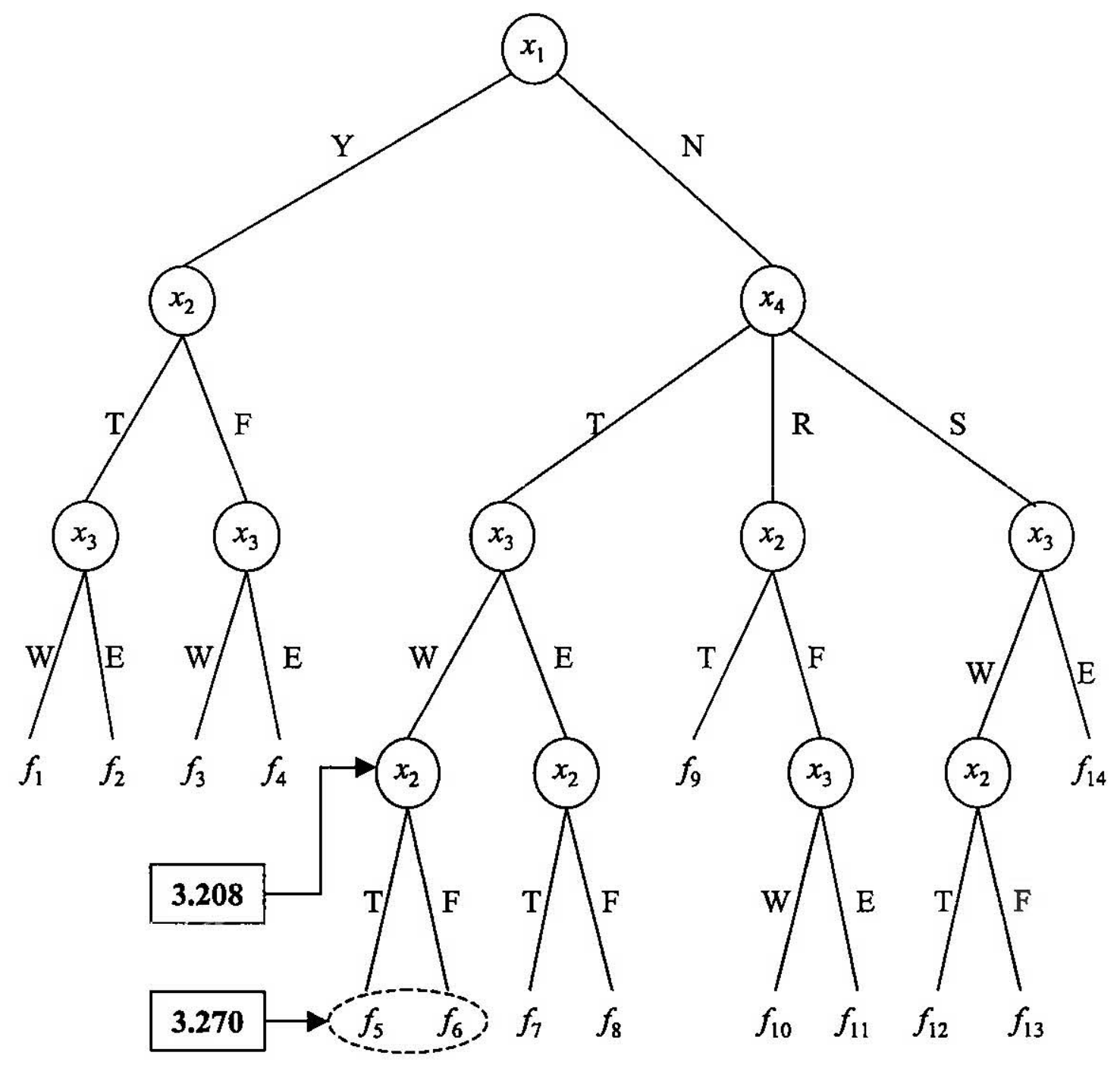

Fig. 3.4 Pruning process for the example in Section 3.3.1. 


\section{CHAPTER 4}

\section{EVALUATION OF EDDE}

Machine learning is one of the most experimentally oriented subfields within artificial intelligence. After a system is built, there is a need to understand the behavior of the system through an experimental study. The experimental study of the system, in other words, testing of the method, should be carried out on both synthetic domains and real world domains [Langley, 1996b]. Experiments with artificial data have important roles in studying the behavior of a system. Such data sets let one systematically vary factors of interest, such as the number of variables, the number of examples, and the amount of noise. By systematically varying the synthetic domain characteristics of the data set, the effect of these factors on the system behavior can be measured and the various aspects of the system are hopefully understood. In this section, an extensive experimental study of EDDE's performance on artificial data is provided.

\subsection{TREE INSTABILITY}

An experiment is carried out on a series of data sets generated in exactly the same way, but each with a different random number seed. Induced trees and thus their corresponding region-equation pairs may be different under the same model formation parameters. Even for the same data set, when the test sets are selected randomly with different random number seeds, induced trees may also be different. 
corresponding region-equation pairs are said to differ from the known model. The induction of false trees is referred to as tree instability.

The amount of tree instability is affected by the noise in the data. The possible causes of instability can be tracked down in the induction process. First, in selecting significant variables for region description, there may be a number of splits on different variables at a given node, some of which give almost the same error reduction. Since data are noisy, the choice between competing splits is almost random. Choosing an alternative split that is almost as good will led to a different evolution of the tree from that node downward. This kind of instability happens quite often in other tree-based systems such as CART [Breiman, Friedman, Olshen and Stone, 1984], but is much less severe in EDDE. Various tests have shown that this kind of instability happens after all significant description variables have been identified, which means different tree structures happen at the lower level of trees (see Fig. 4.1 (b) and (c)). This is attributed to the introduction of domain knowledge, which is used to divide variables into description variables and prediction variables before building a tree. EDDE makes use of domain knowledge to get more stable results whereas CART and the like do not. This shows the importance of introduction of domain knowledge.

Second, in eliminating insignificant variables from a region equation, a variable is eliminated if it does not give an improvement in standard deviation beyond the threshold $\delta$. Because of the clear-cut threshold for elimination, sometimes the significant variable may be eliminated (see Fig. 4.1 (c) $f_{5}$ ) and at other times insignificant variables may not be removed from the equation (see Fig. 4.1 (d) $f_{1}$ ). This will also lead to a false tree.

Third, the instability comes from the pruning process after a tree has been constructed. During the pruning process, a tree is pruned based on the error measure on testing data. If the subtree of a node does not improve the prediction on testing data more than the threshold $\varepsilon$, the subtree will not be pruned. Because of noisy data 


\subsubsection{The Influence of the Number of Variables}

As mentioned in Chapter 1, one of the characteristics of real world engineering problems is that engineers can not always determine the significance of variables a priori. In the following experiment, we investigate how insignificant (or irrelevant) variables affect tree stability ratio.

This experiment also uses Model 1 in Section 3.3 to generate data sets, but more insignificant variables are introduced in this experiment. The number of insignificant variables included in the data varies from 6 to 46 in increments of 8 . Therefore, the total number of the variables including 4 significant variables in the model varies from 10 to 50 in increments of 8 , that is $10,18,26,34,42$, and 50 . Of the insignificant variables, half of them are description variables and the other half are prediction variables. Of the insignificant description variables, half of them have two evenly distributed values like $x_{1}$ in Model 1; the other half have three evenly distributed values like $x_{4}$ in Model 1 .

As mentioned, the influence of the number of variables might be interactive with sample size and noise variance. Therefore, tree stability ratio versus the number of variables is studied when both of the sample size and the noise variance are kept at certain levels. The sample size varies from 200 to 800 in increments of 200 , that is: $200,400,600$, and 800 . The noise variance varies from 10 to 500 , that is 10,100 , $200,300,400$, and 500. The selection of these variances is explained in Section 4.2.3. The tree stability ratio is investigated under all the combinations of these sample sizes and noise variances.

The tree stability ratios versus the number of variables under various noise variances are shown in Fig. 4.2 to Fig. 4.7. The figures show that tree stability ratio does not change much as the number of variables increases. For example, when the sample size is 100 and noise variance is 10 , the stability ratio remains approximately 95 when the number of variables ranges from 10 to 50 . Tree stability ratio slightly decreases with respect to increase in number of variables only when noise variance is 
$600,800,1400$, and 2000. Data sets of various sample sizes $(200,400,600,800$, 1400 , and 2000) at a fixed noise variance are generated by MATLAB based on the Model 1.

Section 4.2.1 also shows that the influence of sample size is related to noise variance. To more thoroughly study the influence of sample size, the dependence of stability ratio on sample size at various noise variances are also examined. The noise variances selected for study range from 10 to 500 , that is $10,100,200,300,400$, and 500. The seiection of these variances is expiained in Section 4.2.3.

The influence of sample size on stability ratio is shown in Fig. 4.12 when noise variance is also taken into account. The figures show that sample size has influence on tree stability ratio. Under the same level of noise variance, smaller sample sizes result in lower stability ratios. When sample size increases, induced trees become more stable. At the first stage, the stability ratio increases sharply, then its increase gradually slows down. With large sample size, induced trees eventually become stable. However, at what sample size the induced trees become stable depends on the noise variance. When variance is 100 , the sample size of 800 makes the induced tree stable. But the sample size of 800 will not make the induced tree stable when noise variance is 500. The influence of noise is discussed in Section 4.2.3.

\subsubsection{The Influence of Noise}

The influence of introduced noise on stability is studied in this section. Since the number of variables does not influence stability ratio as known from Section 4.2.1, the number of variables is kept the same in the investigation. Model 1 in Table 3.1 is again used to generate data sets with the number of variables equal to 10 .

In order to determine the influence of noise, stability ratios of data sets corrupted with various noise variances are studied. Noise variances are selected as $10,100,200,300,400$, and 500 . The reason to select these noise variances less than 
unnecessary tree structure. Taking the experiment results in Section 4.1 as an example, the true tree (Fig. 4.1 (a)) has higher prediction strength than the false trees (Fig. 4.1 (b), (d) and (e)). Also, the true tree (Fig. 4.1 (a)) has the comparable prediction strength without unnecessary tree structure (Fig. 4.1 (c)). This observation promotes a method to stabilize the final induced tree and the final model.

To deal with the instability of model trees and find the true function, the system EDDE runs the data a user preset number of times (called Repeat in the system) by randomly selecting the test data with different random number seeds. EDDE will induce a model tree each time and choose the one with the consideration of the trade off between the prediction error on test data and model complexity. In fact, if the induced model captures the actual regularity in data, it is entirely possible that the learned model will be the same as the true model.

However, it is hard to find an optimal value of Repeat for building trees because many factors influence the stability of the final reported tree as seen in Section 4.2. Qualitatively speaking, the larger the Repeat, the lower the learning speed and the higher the probability of discovering actual regularity. The default value for repeat is 20 . Users have easy access to adjust this value based on sample size and noise strength.

\subsection{COMPARISON STUDY}

The experiment in this section compares the results given by CART [Breiman, Friedman, Olshen and Stone, 1984] and other algorithms [Quinlan, 1993] and the results by EDDE in order to investigate the model tree's interpretation strength and prediction strength. CART is a regression tree method. The method used in Quinlan's study [1993] consist of one default method, one instance-based method, three model-based methods (regression, model tree, and neural nets), and combinations of the instance-based method with each model-based method. The experiment is carried out on the data set generated in the exactly the same way as that 
reported equation is

$$
\begin{array}{r}
\text { if } x_{1}=1, y=3.170+3.044 x_{2}+1.800 x_{3}+0.891 x_{4} \\
<\mathrm{Sd}=1.530, \mathrm{COD}=0.797> \\
\text { if } x_{1}=-1, y=-2.972+2.910 x_{5}+1.675 x_{6}+1.201 x_{7} \\
<\mathrm{Sd}=1.483, \mathrm{COD}=0.814>
\end{array}
$$

with

$$
\begin{array}{ll}
\text { Model size }(m s) & =2 \\
\text { Leaf size }(l s) & =3 \\
\text { Prediction error }(e) & =1.02 \\
\text { RE } & =0.11
\end{array}
$$

However, on the simulated data by the same known model, CART's regression tree is much larger with 13 leaves. The prediction in each leaf is the dependent variable mean of the cases in that leaf. The induced tree is shown in Fig. 4.14 (b).

Table 4.2. Error measure comparison on the evaluation data.

\begin{tabular}{|l|c|c|}
\hline & EDDE & CART \\
\hline $\begin{array}{l}\text { The number of leaves } \\
\text { (model size } m s \text { ) }\end{array}$ & 2 & 13 \\
\hline Leaf size $(l s)$ & 3 & -- \\
\hline Prediction error $(e)$ & 1.14 & --- \\
\hline RE & 0.12 & 0.17 \\
\hline 1-RE & 0.88 & 0.83 \\
\hline
\end{tabular}

CART provides only the estimate of relative error RE on the evaluation data (the additional 5000 cases), which is defined in Eq. (3.18). The comparison of the induced trees by EDDE and CART is listed in Table 4.2.

From the comparison in Table 4.2 and Figure 4.14, it is noticed that while EDDE's function divides the data space into two regions as does the known function, 
7. neural nets: a straight forward neural network was constructed and trained using the default conjugate gradient method of the Xerion neural network simulator developed at the University of Toronto,

8. neural nets + instances: the IBL approach is combined with the neural net.

Quinlan studied the prediction strength of these methods on the simulated data using the same known model as Model $2^{1}$. The performance of these methods is measured by 10 -fold cross-validation. To compare the results with EDDE, the 200 cases are divided into 10 subsets. For each run, only 9 subsets are provided to EDDE leaving the other subset as evaluation data. Therefore, there are 10 runs. The average error measures on the evaluation data of the 10 runs are listed in Table 4.3.

Table 4.3 shows that the prediction error $(e)$ and the relative error (RE) are 1.18 and $12 \%$ by EDDE respectively. (The percentage error is not compared because the actual values of the dependent variable $y$ include zero). They are comparable to or lower than all the algorithms presented in [Quinlan, 1993] except Quinlan's model tree method. Apparently, EDDE outperforms most of algorithms, with the improvement rate of up to $220 \%$ in prediction error and $180 \%$ in RE. This is not a surprise because those algorithms do not require any information on the domain and no domain knowledge is provided to the system. In contrast, EDDE was given the additional information that $x_{1}, x_{8}, x_{9}$, and $x_{10}$ are used for region description and the other variables are used for region prediction. This demonstrates the important role of domain knowledge in knowledge discovery from data. With the introduction of domain knowledge, the system can discover equations with higher prediction strength. This is seen for EDDE's performance on actual data sets, as discussed in the

'Quinlan's 1993 study uses a total of eight data sets to compare the eight methods. EDDE is here run on the single artificial data set generated by Model 2. In Section 5.5, EDDE is run on the actual data set dealing with automobile fuel consumption. The other 6 actual data sets (housing, cpu, auto-price, servo, lhrh-att, and lhrh-def) are not run on EDDE because domain knowledge is required for EDDE and this knowledge is not included in the data sets. 
up to 300. However, actual engineering data sets are often small so that tree instability occurs.

To deal with the instability of model trees and find the tree equations, a method, using trade-off parameters to select the final tree among alternate trees, is promoted to stabilize the final induced tree. Using the method even on a relatively small sample size (200 with 10 variables), the system can find the actual equations from the data, which is shown when the tree interpretation and prediction strength are studied and compared with other methods [Quinlan, 1993] in Section 4.4. The other methods includes regression tree CART, instance based IBL, multivariant linear regression, model trec M5, neural nets, and combinations of these methods.

On the same data set, EDDE generates the equations that divide the data space into two regions as does the known function while CART's equations divides the data space into 13 regions. The results show that EDDE's induced tree summarizes the data more concisely, and its description of the data is more straightforward comparing with CART.

Also on the same data set, the prediction strength is compared with other methods in [Quinlan, 1993]. The results show that the prediction error is 1.18 by EDDE while the prediction error ranges from 1.1 to 3.77 by other methods. Apparently, EDDE outperforms most of the algorithms, with the improvement rate of up to $220 \%$ in prediction error and of up to $180 \%$ in RE. 


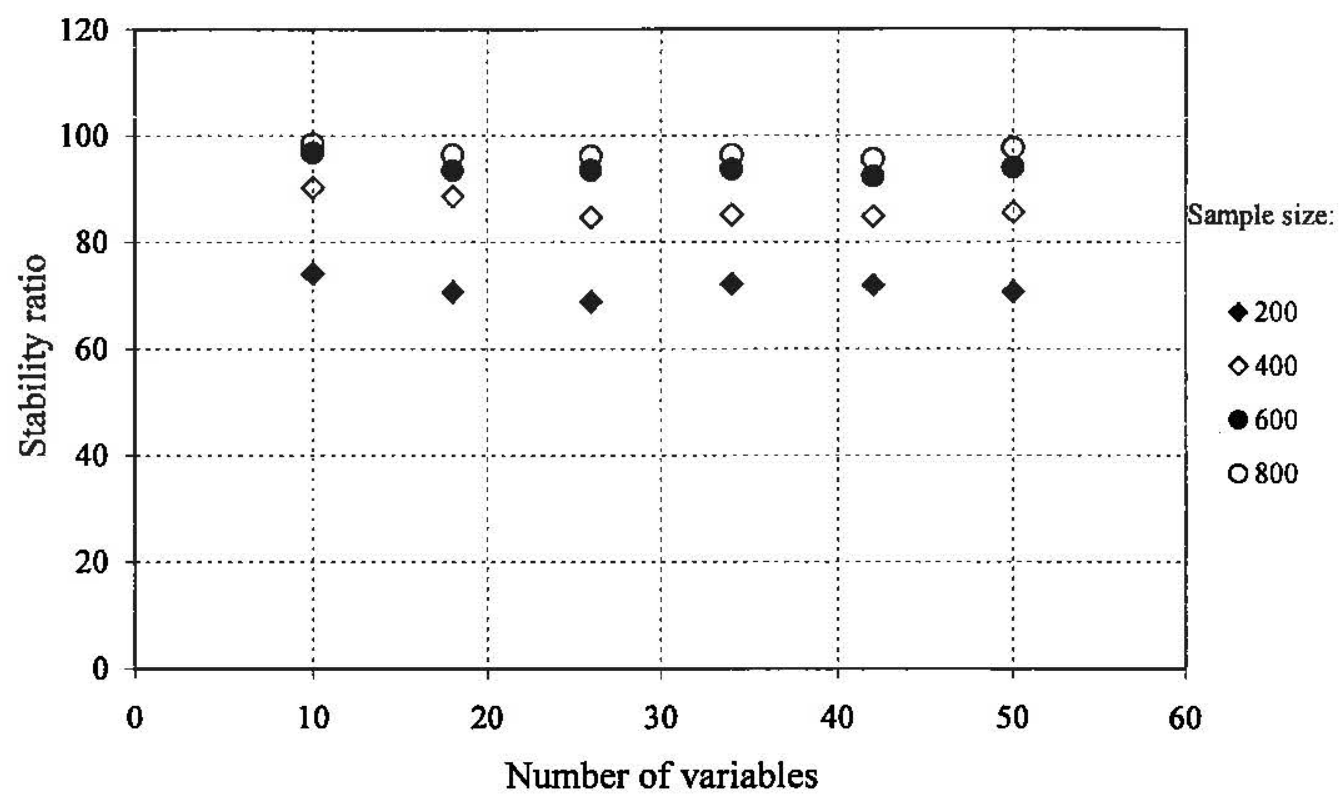

Fig. 4.2 Stability ratio when noise variance is 10 .

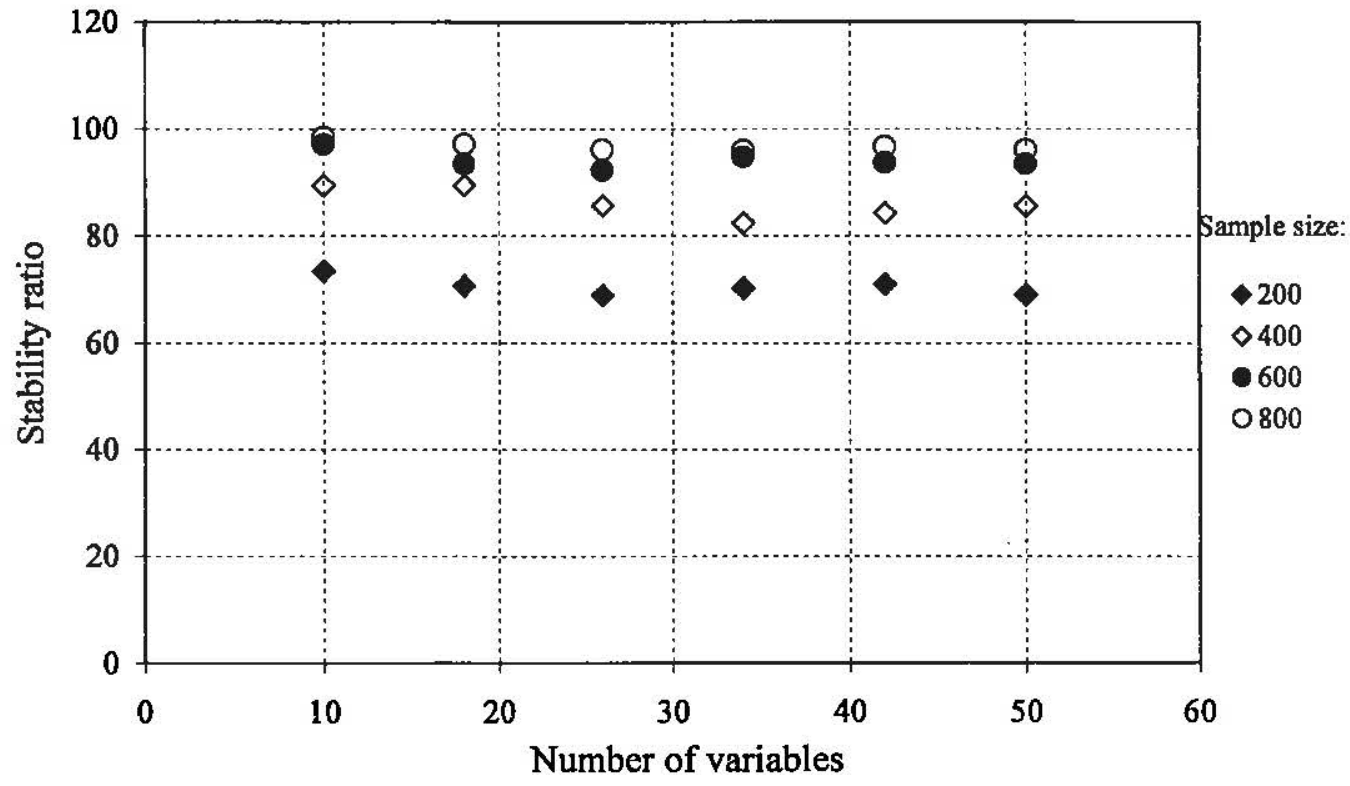

Fig. 4.3 Stability ratio when noise variance is 100 . 


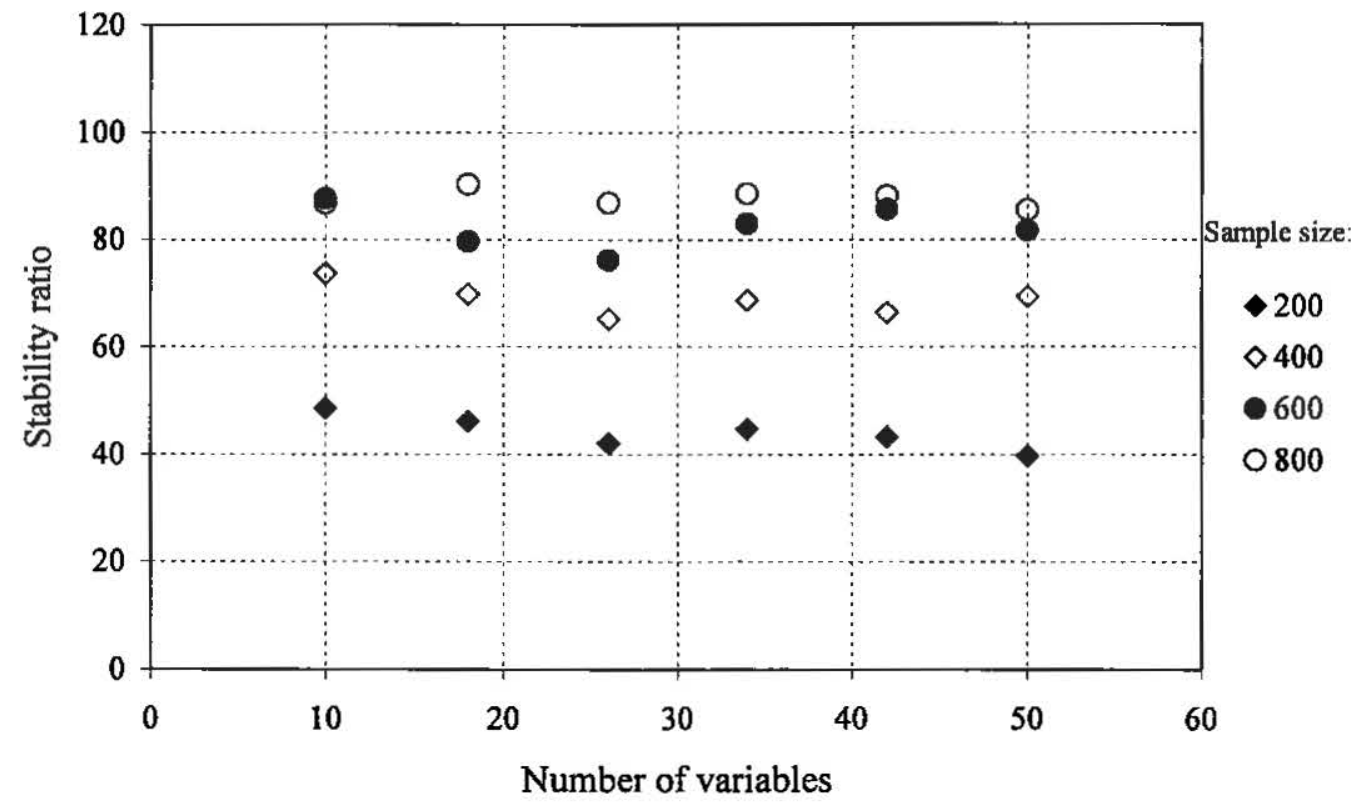

Fig. 4.6 Stability ratio when noise variance is 400 .

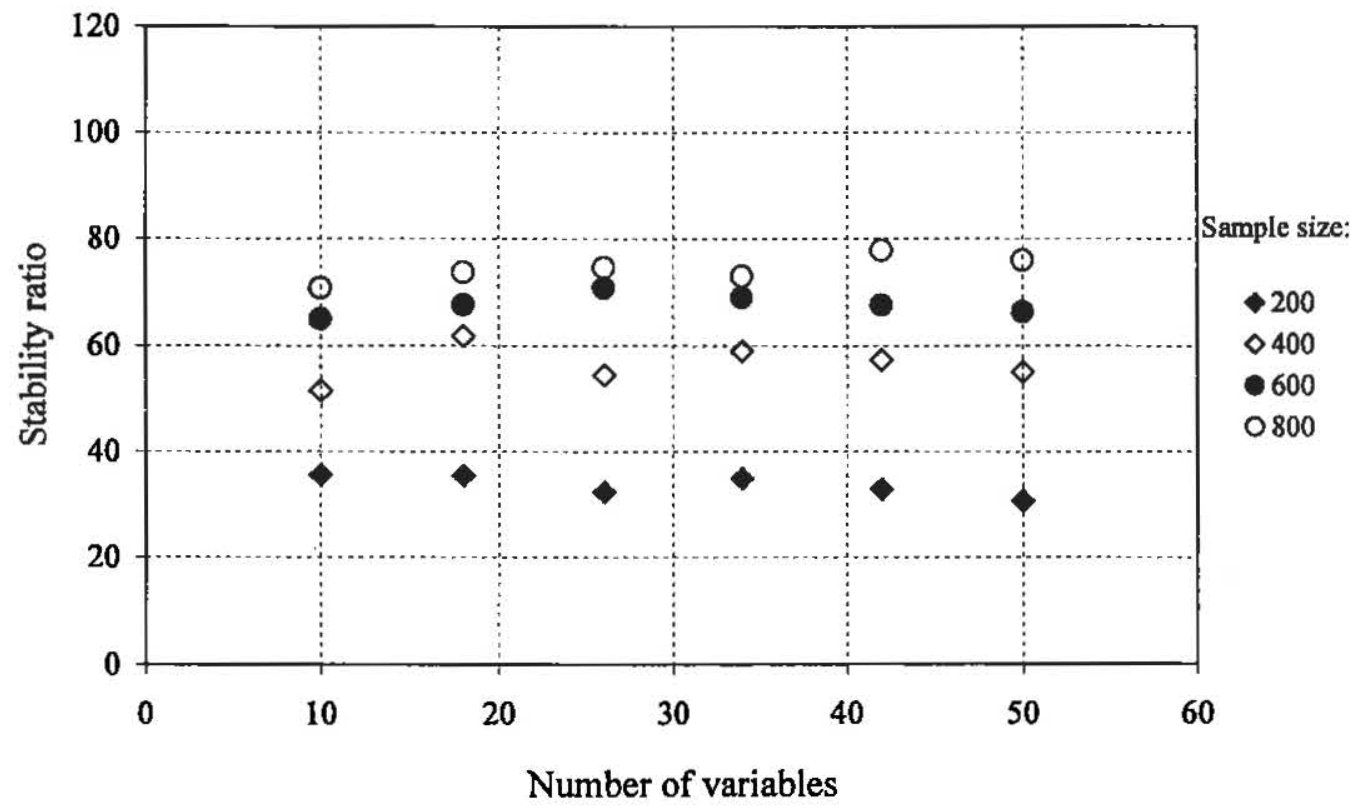

Fig. 4.7 Stability ratio when noise variance is 500 . 


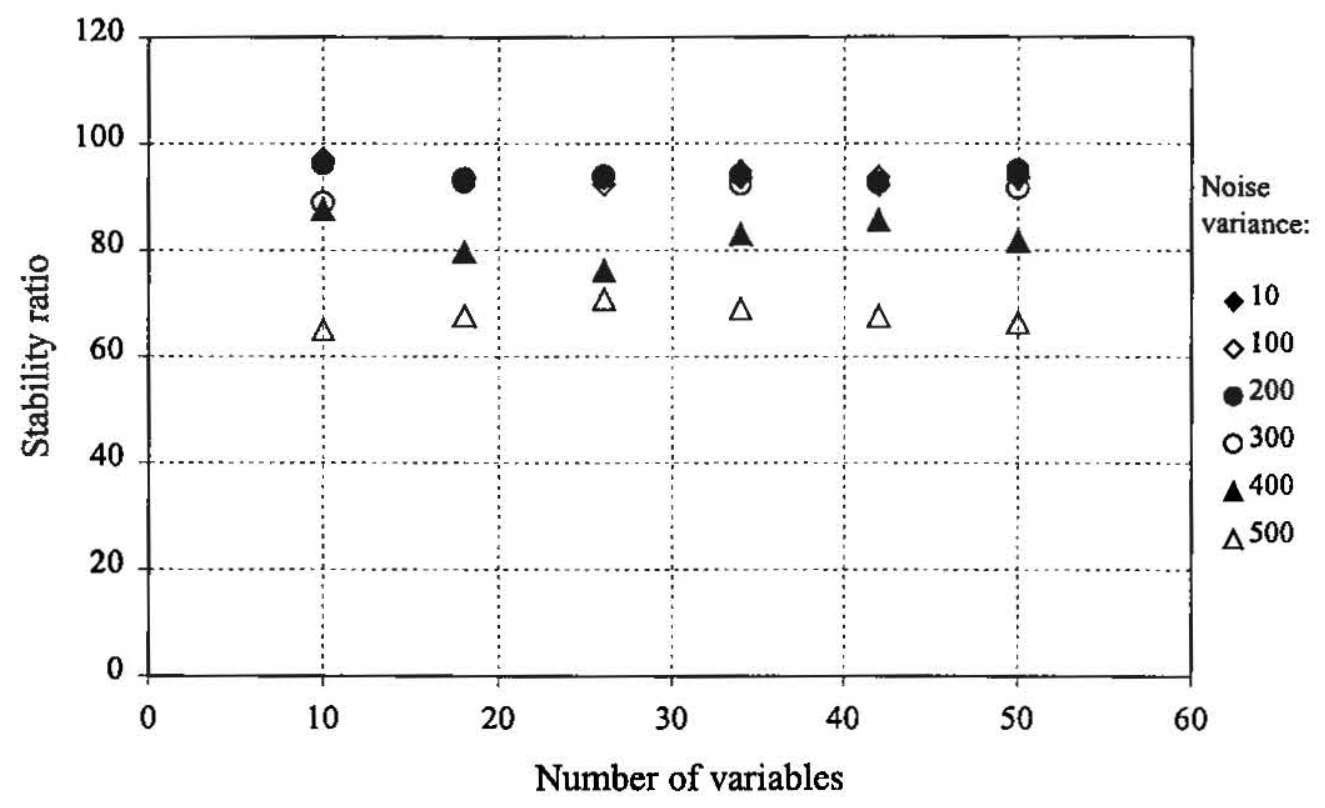

Fig. 4.10 Stability ratio when sample size is 600 .

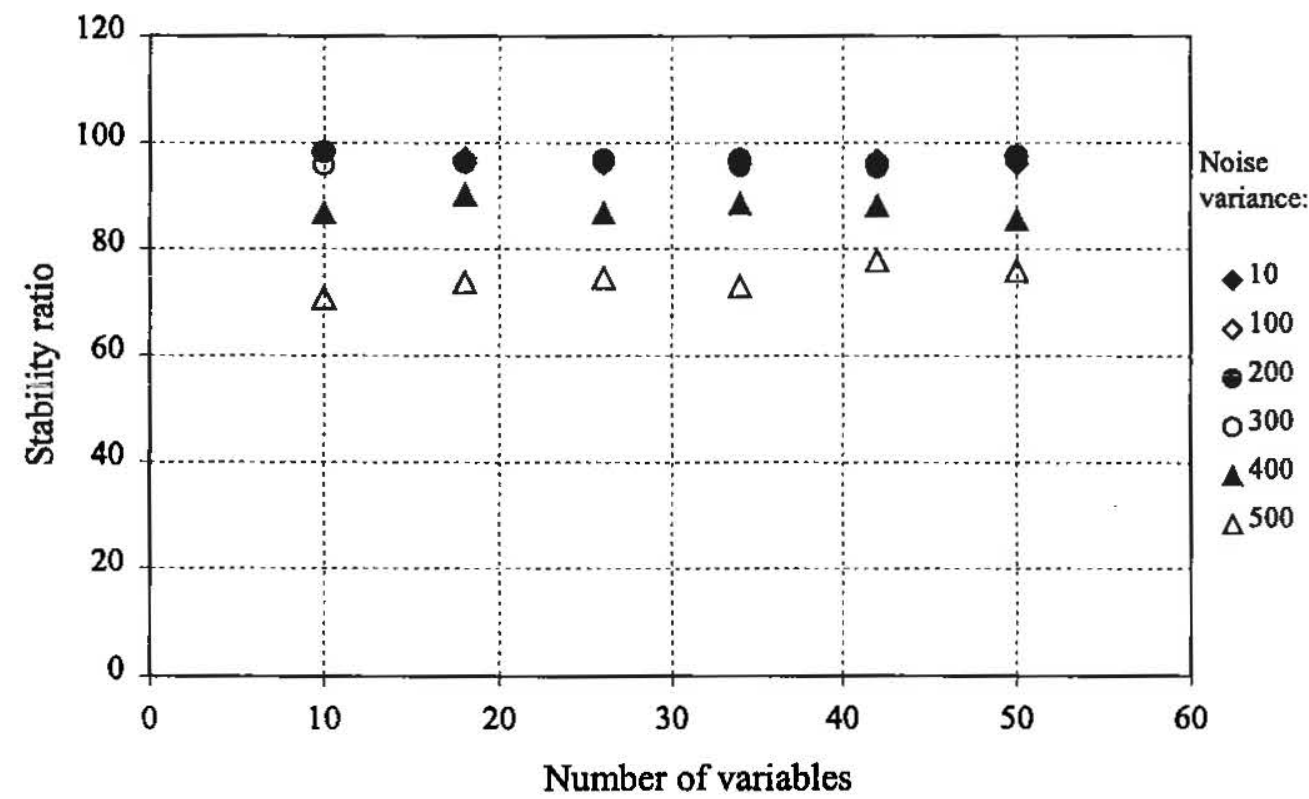

Fig. 4.11 Stability ratio when sample size is 800 . 


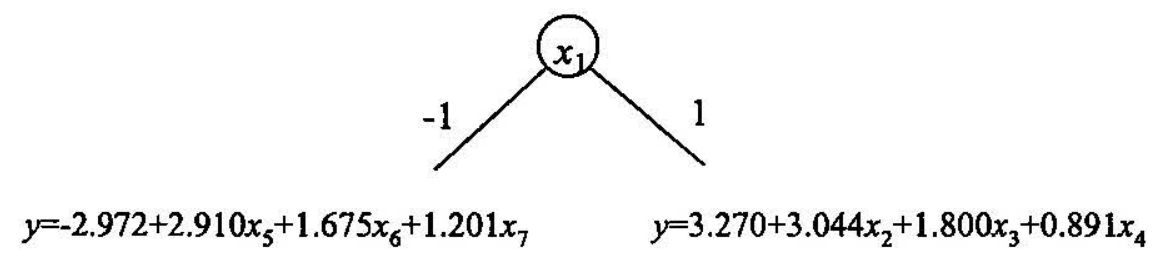

(a) EDDE's induced tree

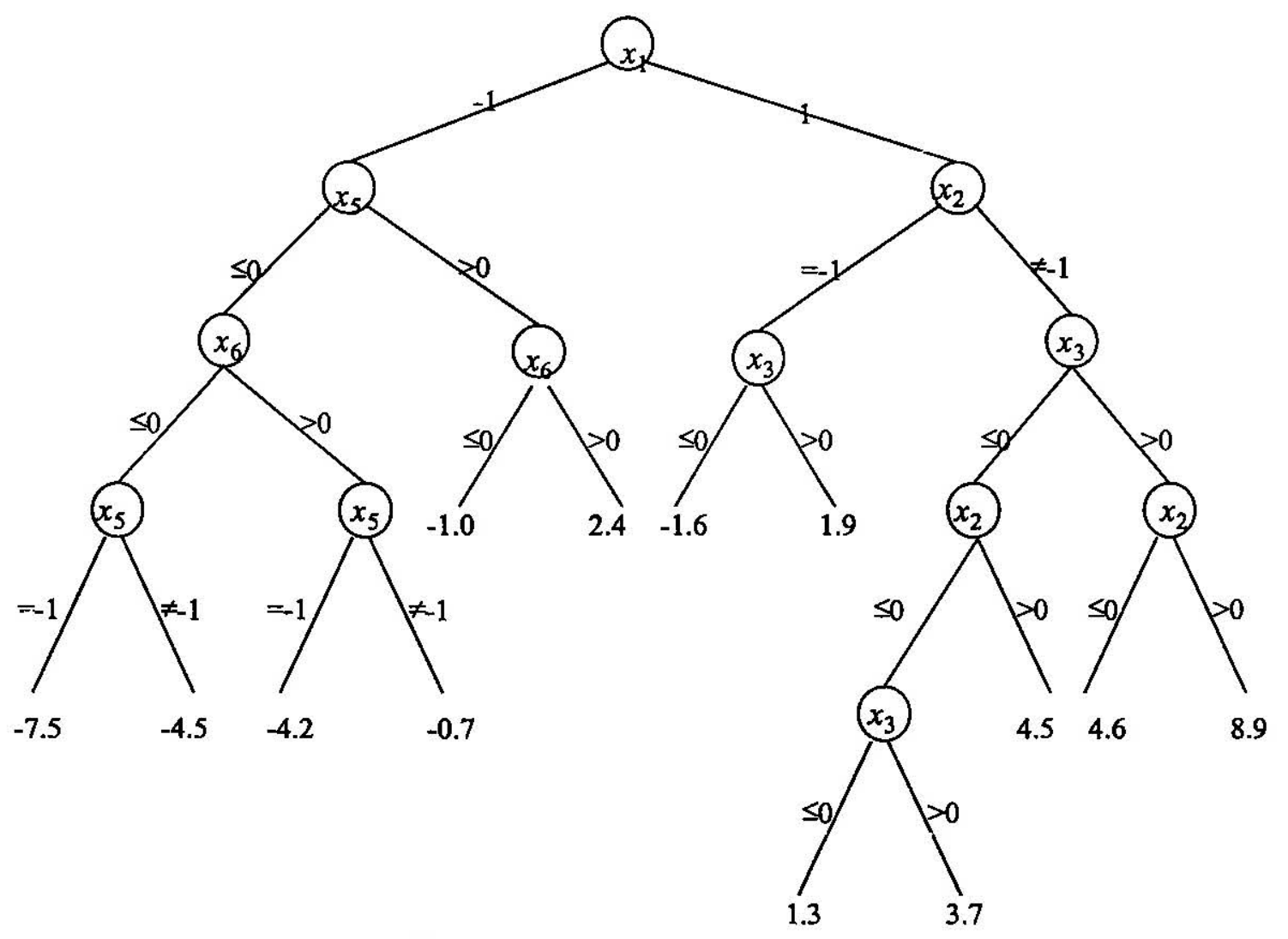

(b) CART's induced tree

Figure 4.14 Induced trees by EDDE and CART for Model 4.2 
project. The planner chooses a generic planning template that most closely matches the project type, establishing an activity network for the project. Next, the duration of each activity in the project is projected according to the predictive models stored in the management system. Finally, a complete plan and schedule is generated either by forward or backward pass calculations.

The effectiveness of planning and scheduling depends on the prediction accuracy of activity duration, which in turn depends on the prediction models. However, the current predictive models were established by experienced engineers based on rules-of-thumb, and their prediction does not accurately reflect the agency's current business practices and requirements. The early phases of EDDE's development were concerned with using the collected historical data to update the predictive models, which are expressed as equations. The updated models result in more effective planning so that costs overruns and schedule slippage can be avoided.

The earlier phases of this research include using the prototype version of EDDE to update the predictive models. Comparing the updated predictive models and the old predictive model, the percentage deviation is $50.7 \%$ using the updated predictive models, while the percentage deviation is $67.5 \%$ using the old predictive model. The updated predictive model does improve the percentage deviation of the duration prediction by $25.4 \%$. The induced equations are of practical value, which displays the usefulness of the research presented in this thesis. Meanwhile, it was also learned that the quality of the induced equations highly depends on the quality of the provided data set. When data sets are corrupted with strong noise, the data sets have to be cleaned and preprocessed. The issues of data cleaning and preprocessing should be addressed in the data preparation step of the whole process of knowledge discovery in databases, which is beyond the scope of the study presented in this thesis. The earlier phases of research and results were presented at the 1997 Transportation Research Board annual meeting and published in [Zhang and Roddis, 1997]. More extensive description is provided in [Roddis and Zhang, 1997]. 
and Darwin [1998]. To investigate the design criteria, the study is based on the assumption that the total force in a bar at splice failure, $T_{b}$, equals the sum of a concrete contribution, $T_{c}$, and a transverse reinforcement (steel) contribution, $T_{s}$, which can be written as

$$
T_{b}=T_{c}+T_{s}
$$

In their research, they study both the concrete contribution $T_{c}$ and the transverse reinforcement (steel) contribution $T_{s}$. However, only the concrete contribution $T_{c}$ is studied by EDDE in this section. Therefore, only their results concerning the concrete contribution $T_{c}$ are provided below.

Using a database including 171 specimens containing developed or spliced bars not confined by transverse reinforcement, the contribution of $T_{c}$ is studied. (The data set is listed in Appendix B.) $T_{c}$ is the product of the bar area, $A_{b}$, and the bar stress at failure, $f_{s}$, in psi. They study the relationship between $\frac{T_{c}}{f_{c}^{\prime p}}\left(=\frac{A_{b} f_{s}}{f_{c}^{\prime p}}\right)$ and specimen properties of the beam, where $p$ is a constant, and $f_{c}^{*}$ is concrete compressive strength in psi.

A series of dummy variable analyses are carried out based on bar size and concrete strength when different power values of $p$ and effective $c_{s i}$ are considered. The findings of their research include but are not limited to the following:

- When $p=1 / 4$ and effective $c_{s i}=$ actual $c_{s i}+0.25$, the introduction of the term

$$
\begin{gathered}
\left(0.1 \frac{c_{\max }}{c_{\min }}+0.9\right) \text { is necessary to describe } \frac{T_{c}}{f_{c}^{\prime p}} \text { and the equation is expressed as } \\
\frac{T_{c}}{f_{c}^{1 / 4}}=\left[59.81 l_{d}\left(c_{\min }+0.5 d_{b}\right)+2350 A_{b}\right]\left(0.1 \frac{c_{\max }}{c_{\min }}+0.9\right)
\end{gathered}
$$

where

$$
c_{\min }, c_{\max }=\text { minimum and maximum values of } c_{s} \text { and } c_{b}
$$


process. However, to accommodate users' different needs, EDDE provides other information about the discovered equations. The additional information, which is described in Section 3.3.3, includes the ratio mean and COV. But, ratio mean and COV in Zuo's research and in EDDE are calculated differently. Ratio mean and COV in Zuo's research are the resubstitution estimations of all input data, while those in EDDE are the sample estimations of only testing data. Obviously, Zuo's error measures may overestimate the equation performance in comparison to EDDE error measures because Zuo's estimations of ratio mean and COV are computed using the same data used to establish the equation.

\subsubsection{Data Preparation}

To test EDDE on this actual engineering data set including 171 examples, the data set is prepared before the actual learning process starts. A part of data, suggested by a domain engineer and containing 28 examples (from Zuo's experiments [Zuo, 1998]), is held as evaluation data, which will not be given to EDDE for learning. The other part containing 143 examples is given to EDDE to discover equations. After equations are discovered, the evaluation data is used to evaluate the equations.

On the 143 data points, the following is done.

delete 2 data points with $d_{b}=0.375 \mathrm{in}$.

delete 5 data points with $d_{b}=0.625$ in.

delete 1 data points with $d_{b}=1.128$ in.

delete 2 data points with $d_{b}=1.177$ in.

delete 2 data points with $d_{b}=1.960 \mathrm{in}$.

change 2 data points with $d_{b}=0.992 \mathrm{in}$. to $d_{b}=1.000 \mathrm{in}$.

The reason for the changes is that EDDE requires the number of each data subset to be larger than the number of the variables when EDDE tests whether to use a variable to divide the domain space. If those data are kept, the test for variable $<$ bar size $>$ will fail and the influence of $<$ bar size $>$ as a possible variable for splitting the data into subsets will not be checked. 
area of the reinforcing bar $A_{b}$.

Third, experiments also show that $c_{\max }$ and $c_{\min }$ affect $T_{c} . T_{c}$ becomes larger as the ratio $c_{\max } / c_{\min }$ increases. This was found by plotting the version of equation 5.2 that does not account for $c_{\max }, \frac{T_{c}}{f_{c}^{1 / 4}}=$ $59.81 l_{d}\left(c_{\min }+0.5 d_{b}\right)+2350 A_{b}$, for various values of $c_{\max }$ and observing the upward trend in $T_{c}$ with increasing $c_{\max }$. To account for this behavior of the data, the term $\left(0.1 \frac{c_{\max }}{c_{\min }}+0.9\right)$ was introduced in Eq. 5.2.

Based on the above considerations, the following basic templates are selected for submittal to EDDE.

a. $l_{d}\left(c_{\min }+0.5 d_{b}\right)$

b. $A_{b}$

c. $l_{d}\left(c_{\min }+0.5 d_{b}\right)\left(0.1 \frac{c_{\max }}{c_{\min }}+0.9\right)$

d. $\quad A_{d}\left(0.1 \frac{c_{\max }}{c_{\min }}+0.9\right)$

- The descriptive variables are

a. Bar size, represented by bar diameters

b. Concrete strength, discretized based on the study by Zuo and Darwin [1998] as

$$
\begin{aligned}
& 2500 \mathrm{psi}<f_{c}^{\prime} \leq 3500 \mathrm{psi} \\
& 3500 \mathrm{psi}<f_{c}^{\prime} \leq 4500 \mathrm{psi} \\
& 4500 \mathrm{psi}<f_{c}^{\prime} \leq 5500 \mathrm{psi} \\
& 5500 \mathrm{psi}<f_{c}^{\prime} \leq 6500 \mathrm{psi} \\
& 6500 \mathrm{psi}<f_{c}^{\prime} \leq 14500 \mathrm{psi}
\end{aligned}
$$


that $\frac{T_{c}}{f_{c}^{\prime 1 / 2}}$ is described by a different equation for each range of $f_{c}^{\prime}$. For example, when $60 \%$ of data is used as testing data, the induced equations are

$$
\begin{aligned}
& \text { if } f_{c}^{\prime}=2500 \sim 3500 \\
& \frac{T_{c}}{f_{c}^{1 / 2}}=\left(8.545 l_{d}\left(c_{\min }+0.5 d_{b}\right)+287.171 \mathrm{~A}_{\mathrm{d}}\right)\left(0.1 \frac{c_{\max }}{c_{\min }}+0.9\right) \\
& \text { if } f_{c}^{\prime}=3500 \sim 4500 \\
& \frac{T_{c}}{f_{c}^{1 / 2}}=\left(5.118 l_{d}\left(c_{\min }+0.5 d_{b}\right)+467.105 \mathrm{~A}_{\mathrm{d}}\right)\left(0.1 \frac{c_{\max }}{c_{\min }}+0.9\right) \\
& \text { if } f_{c}^{\prime}=4500 \sim 5500 \\
& \frac{T_{c}}{f_{c}^{1 / 2}}=\left(8.280 l_{d}\left(c_{\min }+0.5 d_{b}\right)+190.730 \mathrm{~A}_{\mathrm{d}}\right)\left(0.1 \frac{c_{\max }}{c_{\min }}+0.9\right) \\
& \text { if } f_{c}^{\prime}=5500 \sim 6500 \\
& \frac{T_{c}}{f_{c}^{1 / 2}}=\left(6.871 l_{d}\left(c_{\min }+0.5 d_{b}\right)+266.329 \mathrm{~A}_{\mathrm{d}}\right)\left(0.1 \frac{c_{\max }}{c_{\min }}+0.9\right) \\
& \text { if } f_{c}^{\prime}=6500 \sim 14500 \\
& \frac{T_{c}}{f_{c}^{\prime 1 / 2}}=\left(3.205 l_{d}\left(c_{\min }+0.5 d_{b}\right)+322.297 \mathrm{~A}_{\mathrm{d}}\right)\left(0.1 \frac{c_{\max }}{c_{\min }}+0.9\right) \\
& \text { if } f_{c}^{\prime}=14500 \sim 16500 \\
& \frac{T_{c}}{f_{c}^{1 / 2}}=\left(572.266 \mathrm{~A}_{\mathrm{d}}\right)\left(0.1 \frac{c_{\max }}{c_{\min }}+0.9\right)
\end{aligned}
$$

Zuo [Zuo and Darwin, 1998] concludes that $p=1 / 2$ can not represent the effect of concrete strength on the development/splice strength of bars with a single equation. That is to say, the equations describing $\frac{T_{c}}{f_{c}^{1 / 2}}$ in different ranges of concrete strength should be different. The equations induced by EDDE show this same behavior of different equations for different $f_{c}^{\prime}$ regions. But, the equations in the regions can not 
because the analysis of this data set is sought to get the lowest COV with a ratio mean closest to $1,60 \%$ should be used in the following analysis.

Table 5.1. Discovered equation when the dependent variable is $\frac{T_{c}}{f_{c}^{\prime 1 / 4}}$

\begin{tabular}{|c|c|c|c|c|c|c|}
\hline $\begin{array}{l}\text { percentage } \\
\text { of testing } \\
\text { data }\end{array}$ & $K_{1}$ & $K_{2}$ & $\mathrm{R}^{2}$ & $e$ & $\begin{array}{l}\text { Ratio } \\
\text { Mean }\end{array}$ & COV \\
\hline 10 & 51.746 & 2793.081 & 0.9718 & 181.663 & 0.9872 & 0.0903 \\
\hline 20 & 52.557 & 2715.439 & 0.9730 & 346.435 & 1.0282 & 0.0934 \\
\hline 30 & 50.242 & 2929.775 & 0.9728 & 354.173 & 0.9679 & 0.1209 \\
\hline 40 & 50.367 & 2919.484 & 0.9705 & 307.719 & 0.9845 & 0.1189 \\
\hline 50 & 54.336 & 2615.943 & 0.9717 & 380.349 & 1.0261 & 0.0934 \\
\hline 60 & 52.226 & 2799.449 & 0.9683 & 360.582 & 0.9856 & 0.0893 \\
\hline 70 & 50.985 & 2854.187 & 0.9694 & 343.735 & 0.9929 & 0.0949 \\
\hline 80 & 50.171 & 2815.255 & 0.9723 & 400.752 & 1.0123 & 0.1064 \\
\hline 90 & 51.493 & 2789.824 & 0.9648 & 377.982 & 0.9938 & 0.1025 \\
\hline
\end{tabular}

5.2.3.3 Power $p$ in Dependent V ariable $\frac{T_{c}}{f_{c}^{\prime p}}$

The Section 5.2.3.1 and 5.2.3.2 discuss the results when $p=1 / 2$ and $p=1 / 4$ in

$\frac{T_{c}}{f_{c}^{\prime p}}$. In this section, the influence of $p$ is more extensively examined.

The power $p$ is varied from 0.22 to 0.50 . Because different dependent variables are taken into account, the prediction error $e$ on the testing data can not be used as the criterion to decide which one is better than the others. In this test, the ratio mean and the COV of testing data are taken as the main criteria. The results are 
5.4.(b)

To reflect the splitting failure modes for bond due to the effect of concrete cover and bar spacing, effective $c_{s i}$ is studied. In ACI 318-95, the effective value of $c_{s i}$ is equal to $c_{s i}$. In the Canadian code (CSA Standard A23.3-94), a greater value $\left(4 / 3 c_{s i}+1 / 6 d_{b}\right)$ is used as the effective value of $c_{s i}$ to give a better match between test development /splice strength and predicted strength than using the actual value of $c_{s i}$. Zuo and Darwin found that using $c_{s i}+0.25$ (in.) as the effective value of $c_{s i}$ gives a better match between test development/splice strength and predicted strength than using the actual value of $c_{s i}$.

To investigate effective $c_{s i}$ using EDDE, first, the templates including all effective values of $c_{s i}$ are put into the system. These templates are:

$$
\begin{aligned}
& l_{d}\left(c_{\text {min }}(k)+0.5 d_{b}\right)\left(0.1 \frac{c_{\text {max }}(k)}{c_{\text {min }}(k)}+0.9\right) \\
& A_{d}\left(0.1 \frac{c_{\text {max }}(k)}{c_{\text {min }}(k)}+0.9\right)
\end{aligned}
$$

where $k=0.0$ and 0.2 0.4 in increments of 0.02, $c_{\min }(k)$ and $c_{\max }(k)$ are $c_{\min }$ and $c_{\max }$ with effective $c_{s i}=$ actual $c_{s i}+k$. The induced equation is

$$
\begin{array}{r}
\frac{T_{c}}{f_{c}^{1 / 4}}=55.414 l_{d}\left(c_{\min }(0.36)+0.5 d_{b}\right)\left(0.1 \frac{c_{\text {max }}(0.36)}{c_{\text {min }}(0.36)}+0.9\right) \\
+2619.910 \mathrm{~A}_{d}\left(0.1 \frac{c_{\text {max }}(0.40)}{c_{\text {min }}(0.40)}+0.9\right)
\end{array}
$$

Because the equation includes the templates with $k$ value for defining effective $c_{s i}$, the learning cycle starts again using revised template. Using the templates listed in Eq. (5.4), but, each time only the templates with the same definition of effective $c_{s i}$ are introduced, where $k=0$, or 0.2 or $\ldots$ or 0.4 . The induced equations have the form of Eq. (5.3) with different effective $c_{s i}=c_{s i}+k$ 
between confined bars, which, in turn, overestimates the concrete contribution to bond strength. Another disadvantage of using the larger effective value of $c_{s i}$ means that the assumed splitting cracks change from a horizontal plane to a vertical plane for some specimens in which splitting was actually controlled by the clear spacing. Based on these considerations, effective $c_{s i}=c_{s i}+0.25$ is used for both confined bars and bars not confined by transverse reinforcement in Zuo and Darwin's research. Because the test on EDDE is limited to the reinforcing bars without transverse confinement, Table 5.3 can only show that effective $c_{s i}=c_{s i}+0.25$ is one of a family of reasonable choices. To maintain comparability to Zuo and Darwin's research, an effective $c_{s i}=c_{s i}+0.25$ is used in the templates for generating EDDE's induced equation for final evaluation.

\subsubsection{Final Evaluation}

The final induced equation is

$$
\begin{gathered}
\frac{T_{c}}{f_{c}^{1 / 4}}=\frac{A_{b} f_{s}}{f_{c}^{1 / 4}}=\left[52.226 l_{d}\left(c_{\min }+0.5 d_{b}\right)+2799.447 A_{b}\right]\left(0.1 \frac{c_{\max }}{c_{\min }}+0.9\right) \\
\mathrm{COD}=0.968262
\end{gathered}
$$

with the following information

$$
\begin{array}{ll}
e & =360.582 \\
e \% & =7.0068 \% \\
1-\mathrm{RE} & =0.9818 \\
r & =0.98560 \\
\mathrm{COV} & =0.08925
\end{array}
$$

The induced equation is evaluated by its predictive performance on the evaluation data that is not accessed during the learning process. This data set, unseen during learning, contains 28 examples from Zuo's experiments. The comparison is listed in Table 5.4. 
used as a black box: give input and get output. Users need to pay attention to the intermediate results and interact with the system so that they can obtain results they can trust.

\subsection{FRACTURE TOUGHNESS}

Although the total number of structures that have failed by brittle fracture is low, brittle fracture is catastrophic. Fracture mechanics addresses the behavior of materials during brittle fracture. An important measure used in fracture mechanics is the material fracture toughness, which characterizes the fracture behavior of structural materials. The three primary factors that affect the fracture toughness of structural and pressure vessel steels are temperature, loading rate, and constraint. Considerable research has been conducted in the areas of temperature and loading rate effects on fracture toughness. Generally, the fracture toughness of structural steels increases with increasing temperature and decreases with increasing loading rate.

To study the effect of constraint on fracture toughness, experiments were carried out and data were collected at the University of Kansas (KU) [Smith and Rolfe, 1997] and at Oak Ridge National Laboratories (ORNL) [Theiss, Shum and Rolfe, 1994]. Using the collected data, the influence of constraint was studied by Smith and Rolfe [1997]. In this section, EDDE is tested on this actual engineering data set, and the results are discussed and compared with those in their research [Smith and Rolfe, 1997].

\subsubsection{Preparation}

\subsubsection{Data Collection}

An experimental investigation was conducted to study the relative roles of crack depth $(a)$ and crack-depth to width ratio $(a / \mathrm{W})$ on the fracture toughness of an ASTM A533-B steel. The experiments were carried out on three-point bending specimens. The test set-up is shown in Fig. 5.5, where $a$ is crack depth and $\mathrm{W}$ is 
only the toughness CTOD is included in the study. There are two reasons for selecting CTOD as the test objective. First, various types of toughness are related with one another. Understanding one of them is of benefit in understanding the others. Meanwhile, their study has already shown that the effect of crack depth and $a / \mathrm{W}$ ratio on the toughnesses are similar. From the qualitative point of view, they are the same. Therefore, studying one type of toughness will be sufficient for the testing of EDDE. Second, CTOD is one of the most often-used notch toughness measure because the CTOD test method is based on the determination of a critical strain at fracture from a load-displacement record that does not require a stress analysis. Using CTOD, linear-elastic fracture mechanics can be extended into the nonlinear elastic-plastic region.

\subsubsection{Data Preparation}

To test EDDE on this actual engineering data set including 85 examples, the data set is prepared before the actual learning process starts. Keeping the data analysis objectives in mind, the data is prepared so that the results can compare the toughness for specimens with a constant crack depth and varying $a / \mathrm{W}$ ratios, and also compare the toughness for specimens with varying crack depths and constant $a / \mathrm{W}$ ratios. Therefore, the data preparation mainly includes the discretization of these two variables $a$ and $a / \mathrm{W}$.

After consulting a domain engineer (Dr. Rolfe), the discretization is kept the same as in their study. The actual value of crack depth $a$ ranges from 0.08 to 2.0 in, and it is discretized into 3 nominal values $a=0.08,0.4$, and 2.0 in. The ratio $a / \mathrm{W}$ ranges form 0.1 to 0.5 and is discretized into 3 nominal values $a / \mathrm{W}=0.1,0.32$, and 0.5. The results of discretization are also shown in Appendix C.

\subsubsection{Domain Knowledge}

The domain knowledge, acquired from the discussion with the domain 


\subsubsection{Analysis Results by EDDE}

\subsubsection{Discovered Equation When Both $a$ and $a /$ W Are Description Variables}

In this experiment test, the data set of 85 examples is used and the dependent variable is taken as $\ln (\mathrm{CTOD})$. In order to decide what percentage of data will be used as testing data, preliminary analysis is run on the data with all default model formation parameters except the percentage of testing data. The percentage of testing data varies from $20 \%$ to $80 \%$ in increments of $10 \%$, where the two extremes of $10 \%$ and $90 \%$ are not included because the given data set is small. The selection of the percentage is based on the prediction performance on testing data.

Table 5.5 Test resuits

\begin{tabular}{|c|c|c|c|c|}
\hline $\begin{array}{c}\text { Percentage of } \\
\text { testing data }\end{array}$ & $\begin{array}{c}\text { Prediction } \\
\text { error }(e)\end{array}$ & 1-R & Ratio Mean $(r)$ & COV \\
\hline 20 & 0.24975 & 0.9221 & 0.80258 & 1.04281 \\
\hline 30 & 0.25853 & 0.9285 & 0.99685 & 0.17257 \\
\hline 40 & 0.41793 & 0.8721 & 1.02542 & 0.59647 \\
\hline 50 & 0.29773 & 0.8866 & 0.56607 & 5.14652 \\
\hline 60 & 0.40660 & 0.8229 & 0.93358 & 0.50458 \\
\hline 70 & 0.41277 & 0.8349 & 1.09716 & 0.74747 \\
\hline 80 & 0.43492 & 0.8123 & 0.85552 & 1.04247 \\
\hline
\end{tabular}

The results are listed in Table 5.5. From the table, it is noticed that the best results are obtained when the testing data is $30 \%$ of total given data, which is the default value. Therefore, $30 \%$ of the input data will be used as testing data in later data analysis. Next, we discuss the results given by EDDE when the percentage of testing data is $30 \%$.

The results are shown in Fig. 5.8. Both crack depth and $a / \mathrm{W}$ ratio are used to 
itself. When $a$ is $0.08 \mathrm{in}$. and $2.0 \mathrm{in}$., the $a / \mathrm{W}$ ratio is constant. All $a / \mathrm{W}$ ratios are 0.1 when $a$ is 0.08 in, and all $a / \mathrm{W}$ ratios are 0.5 when $a$ is 2.0 in. When the data does not provide information about varying $a / \mathrm{W}$, it is impossible for EDDE for find how the $a / \mathrm{W}$ ratio influences specimen toughness when $a$ varies to 0.08 in. or $2.0 \mathrm{in}$.

\subsubsection{Toughness with Varying Crack Depth $a$ and Constant $a / W$ Ratio}

In order to compare the toughness for specimens with varying crack depths and a constant $a / \mathrm{W}$ ratio, only the $a / \mathrm{W}$ ratio is taken as a description variable while leaving the crack depth $a$ as a prediction variable. The results are shown in Fig. 5.11 when all model formation parameters are set at default values.

Fig. 5.11 shows that when the $a / \mathrm{W}$ ratio are 0.1 and 0.5 , crack depth does affect the specimen toughness. But, when $a / \mathrm{W}$ is 0.32 , the influence of crack depth is not reflected in the induced equation. This does not mean that crack depth has no effect on the specimen toughness. In fact, crack depth may also influence CTOD when $a / \mathrm{W}$ is 0.32 , which is in between 0.1 and 0.5 , because the effect of the $a / \mathrm{W}$ ratio should be continuous and gradual. However, the data provides no information on varying crack depth when $a / \mathrm{W}$ is 0.32 . When $a / \mathrm{W}$ is 0.32 , all specimen crack depths are 0.4 in.

In the following, we discuss the influence of crack depth on the specimen toughness only when the $a / \mathrm{W}$ ratio is 0.1 and 0.5 . When the $a / \mathrm{W}$ ratio is 0.1 , referred to as a shallow crack geometry, $\ln (\mathrm{CTOD})=6.2783+0.0377 \mathrm{~T}-5.259779 a$. When $a / \mathrm{W}$ is 0.5 , referred to as a deep crack geometry, $\ln (\mathrm{CTOD})=2.9124+0.0237 \mathrm{~T}-0.375273 a$. Obviously, increasing crack depth $a$ will decrease the specimen toughness. However, the strength of the influence is different. Crack depth has less effect on the fracture toughness for deep crack geometry ( $a / \mathrm{W}$ is 0.5$)$ than for shallow crack geometry ( $a / \mathrm{W}$ is 0.1 ). For the same crack depth, assume $a$ is 1.0 in., $\ln$ (CTOD) will decrease 5.2594 for shallow crack depth and 0.3753 for dcep crack depth. The magnitude of decrease in $\ln$ (CTOD) is significantly larger for shallow crack depth than for deep 


\subsubsection{Preparation}

Before the tests are carried out on EDDE, the following preparations have been done, which include the discussions of data collection and domain knowledge.

\subsubsection{Data Collection}

An experimental investigation was conducted to study the dissolution of ionizable drugs. The experiments were carried out on a laminar flow device. The test set-up is shown in Fig. 5.15. As shown in the figure, a dissolution medium flows into the flow channel, then passes the compressed pellet of a drug where some drug is dissolved into the medium, finally, the medium with the dissolved drug flows out of the flow channel. During the tests, the steady state drug concentration in laminar flow at a single flow rate was measured using equipment. Then, the drug flux, defined as the dissolution rate of a drug per surface area, is calculated based on drug concentration. Drug flux will be the dependent variable in the study.

Four model drugs are used in the experiments, which are shown in Tables 5.6. The data are listed in Appendix D at the end of this dissertation. Compared with other data sets discussed in this chapter, this data set is relatively small with 64 entries.

Table 5.6 Model drugs

\begin{tabular}{|c|c|c|}
\hline Model Drugs & Solubility $\left(\times 10^{5} \mathrm{M}\right)$ & $\mathrm{pKa}$ \\
\hline Cinnarizine & 0.598 & 7.47 \\
\hline Naproxen & 13.0 & 4.57 \\
\hline Benzoic & 2250 & 4.03 \\
\hline 2-Naphthoic acid & 13.7 & 4.02 \\
\hline
\end{tabular}


account.

The final induced equation is also tested using evaluation data that is not accessible to EDDE. The testing shows the error measures on evaluation data are consistent with the estimated performance predicted by the system. The prediction error, ratio mean, and coefficient of variation are $360.582,0.9856$, and 0.0893 respectively on evaluation data, while the estimates of these measures are 360.232 , 1.0420 , and 0.0711 respectively by EDDE.

It is learned from this application that the final results are not obtained just by doing one batch of learning. The meaningful results are obtained through the interaction between the system and the user.

The application in Section 5.3 studies the effect of crack length $a$ and crack constraint $a / \mathrm{W}$ ratio on fracture toughness. The learning objectives are to compare toughness for specimens with a constant crack depth $a$ and varying $a / \mathrm{W}$ ratios, and to compare toughness for specimens with varying crack depth $a$ and constant $a / \mathrm{W}$ ratios. These objectives are applied to help the user in classifying the variables into description and prediction variables.

1) When the effect of different crack depth $a$ and crack constraint $a / \mathrm{W}$ is investigated, $a$ and $a / \mathrm{W}$ are both classified as description variables. The results show that $a$ and $a / \mathrm{W}$ have interactive effect on fracture toughness CTOD. 2) To compare toughness for specimens with a constant crack depth $a$ and varying $a / \mathrm{W}$ ratios, $a$ is classified as a description variable and $a / \mathrm{W}$ is classified as a prediction variable. The results show that $a$ is used to divide the domain space, and increasing $a / \mathrm{W}$ ratio will decrease the toughness CTOD when $a$ is constant. 3) To compare toughness for specimens with varying crack depth $a$ and constant $a / \mathrm{W}$ ratios, $a / \mathrm{W}$ is classified as a description variable and $a$ is classified as a prediction variable. The results show that $a / \mathrm{W}$ is used to divide the domain space, and increasing crack depth $a$ will decrease the specimen toughness when $a / \mathrm{W}$ is constant. However, the strength of the 
compared with those by Quinlan's study [1993] in Table 5.9. The methods used in Quinlan's study [1993] are described in Section 4.4.2. Table 5.9 shows that the prediction error and the relative error are 2.33 and $17.0 \%$ respectively by EDDE. They are lower than or comparable to the algorithms (except neural nests and neural nets + instances) presented in [Quinlan, 1993]. Apparently, EDDE outperforms most of the algorithms, with the improvement rate of up to $180 \%$ in prediction error and of up to $35 \%$ in RE. This is not a surprise because those algorithms do not require any information on the domain and no domain knowledge is provided to the system. This also demonstrates the important role of domain knowledge in knowledge discovery from data.

Another advantage of the induced equations over the Quinlan's method is that the trend of mpg with model year can be studied using the induced equations.

From all 392 examples, the average values of each attribute are calculated, and they are

$$
w=2977.6 \quad d=194.4 \quad p=104.5
$$

Substitute these average values into the induced equations, the mpg of each year is obtained. The mpg versus year is plotted in Fig. 5.22. Fig. 5.22 shows that $m p g$ has an increase trend with year although mpg of last year is not always less than that of next year.

\subsection{SUMMARY}

This chapter tests the performance of EDDE in engineering domains using actual engineering data sets. The results show that EDDE can be applied in a variety of engineering domains, verifying that the problem type characterized in Chapter 1 occurs widely in engineering. They also show that understanding the domain and introducing function templates to the system based on domain knowledge play a very important role in discovering useful and meaningful knowledge. They also demonstrate the importance of the interaction between the system and the users. In 
parameters are set at default values except that the percentage of testing data varies from $10 \%$ to $90 \%$ in increments of $10 \%$.

Table 5.8 Results by different percentages of testing data.

\begin{tabular}{|c|c|c|c|c|c|}
\hline $\begin{array}{c}\text { Percentage of } \\
\text { testing data }\end{array}$ & $\begin{array}{c}\text { Prediction } \\
\text { error }(e)\end{array}$ & $\begin{array}{c}\text { Percentage } \\
\text { error }(e \%)\end{array}$ & Accuracy & $\begin{array}{c}\text { Ratio Mean } \\
(r)\end{array}$ & COV \\
\hline 10 & 1.85297 & 8.0734 & 84.21053 & 0.96493 & 0.09429 \\
\hline 20 & 1.94019 & 9.0120 & 82.85714 & 1.05255 & 0.20450 \\
\hline 30 & 1.82198 & 8.1414 & 89.69072 & 1.00200 & 0.10336 \\
\hline 40 & 2.12580 & 9.3674 & 79.06977 & 1.00758 & 0.13008 \\
\hline 50 & 2.07802 & 9.1219 & 78.84615 & 1.01711 & 0.12193 \\
\hline 60 & 2.10521 & 9.6459 & 83.79888 & 1.01284 & 0.19395 \\
\hline 70 & 2.29164 & 9.8994 & 77.89474 & 1.05087 & 0.49790 \\
\hline 80 & 2.35314 & 10.1382 & 77.33333 & 1.01116 & 0.20043 \\
\hline 90 & 3.07342 & 13.7061 & 58.84956 & 1.02713 & 0.18373 \\
\hline
\end{tabular}

Note: All induced equations have 13 region-equation pairs where the regions are divided by the attribute <model year $>$.

Table 5.8 shows that the attribute $<$ model year $>$ is always used to divide the domain space, and the equations induced when the percentage of testing data is $30 \%$ give best prediction on testing data. The induced equations are

Year 70: $\quad m p g=54.46+0.0754 d-0.1847 p-0.0028 w-11.2880 d / p$

Year 71: $\quad m p g=43.35-0.0891 p-6.656144 d / p$

Year 72: $\quad m p g=35.90-0.0053 w$

Year 73: $\quad m p g=20.20+0.069067 p-0.006682 w+0.399698 w / p$

Year 74: $\quad m p g=31.23-0.0030 w-6.1964 d / p+0.4337 w / p$

Year 75: $\quad m p g=37.88+0.1437 d-0.0145 w-15.0480 d / p+0.9108 w / p$

Year 76: $\quad m p g=41.52-0.0065 w$

Year 77: $\quad m p g=78.00+0.1792 d-0.4575 p-23.8928 d / p$ 


\subsubsection{Role of Domain Knowled ge}

First, experiments are carried out on the data set that excludes the examples with the attribute $<$ cylinders $>$ of either 3 or 5 in order to check whether the attribute <cylinders> should be used to divide the domain space. Therefore, the data set contains 385 examples.

All model formation parameters are set at default values. The results show that the attribute <cylinders $>$ has never been used to divide the domain space no matter whether nonlinear templates (discussed in last section) are provided to EDDE. This is consistent with the anticipation based on the domain knowledge. Therefore, the data changes are unnecessary. All 392 examples, without removing the examples whose attribute <cylinders $>$ equal 3 or 5 , are given to EDDE in later experiments in order to used more information and acquire better results.

On the data set of 392 examples, experiments are carried out at three stages. At all three stages, the model formation parameters are set at default values.

At the first stage, no domain knowledge is introduced so that all templates used in learning are linearly related with the dependent variable mpg. A set of equations is induced by EDDE where the attribute <model year $>$ is used to divide the domain space into 13 regions. Information on the performance of the induced equations on testing data is provided in the second column of Table 5.1. At the second stage, in addition to the templates in the first stage, a nonlinear template $d / p$ is introduced to EDDE for learning. A set of equations is induced by EDDE where the attribute <model year> is also used to divide the domain space into 13 regions. Information on the performance of the induced equations on testing data is provided in the third column of Table 5.1. At the third stage, in addition to the templates in the second stage, another nonlinear template $w / p$ is introduced to EDDE for learning. A set of equations is induced by EDDE where the attribute <model year> is also used to divide the domain space into 13 regions. Information on the performance of the 
Section 5.5.1.2.

\subsubsection{Data Preparation}

The data concerns city-cycle fuel consumption in miles per gallon (mpg) to be predicted in terms of three multivalued discrete attributes and four continuous attributes. The three discrete attributes are <cylinders $>,<$ model year $>$, and <origin $>$.

The four continuous attributes are <displacement>, <horsepower $>$, <weight $>$, and $<$ acceleration>. The identifications of description variables and prediction variables are very straightforward. The three discrete attributes are used as description variables and the other four continuous attributes are used as prediction variables. The mpg is taken as the dependent variable.

To test EDDE on this actual engineering data set, the data set is prepared before the actual learning process starts. First, there are 6 examples with missing values. They are removed from the data provided to EDDE. The data set without any missing values contains 392 examples and is listed in Appendix $\mathrm{E}$ at the end of this dissertation.

The data list shows that there are only 4 examples when the attribute $<$ cylinders> equals 3 and only 3 examples when the attribute <cylinders> equals 5 . EDDE requires the number of each data subset to be larger than the number of the variables when EDDE tests whether to use a variable to divide the domain space. If those examples are kept, the tests for variable <cylinders> will fail and the influence of <cylinders $>$ as a possible variable for splitting the data into subset will not be checked. Therefore, those 7 examples with <cylinders $>$ of 3 or 5 are removed from the data provided to EDDE. Such changes make the data set contain 385 examples. In fact, we can see later that the changes are unnecessary because <cylinders $>$ is not used by EDDE to divide the domain space. Therefore, the data set including all 392 examples without the removal of the 7 examples is then provided to EDDE for learning in order to use more given information and obtain better results. 
variable to divide the domain space. If all the prediction variables are introduced at the same time, the test for any of the description variables will fail and the influence of a description variable as a possible variable for splitting the data into subsets will not be checked. Therefore, the description variables cannot be introduced at the same time. Based on repetitive tests, it is found that the maximum of two prediction variables can be introduced at that same time so that the influence of description variables can be checked. Because the medium $\mathrm{pH}$ is the most significant variable based on the existing domain knowledge, at the beginning the prediction variables are introduced in the following ways:

- $<$ Medium $\mathrm{pH}>$ and $<$ drug solubility $>$

- $<$ Medium $\mathrm{pH}>$ and $<$ particle size $>$

- $<$ Medium $\mathrm{pH}>$ and $<$ flow rate $>$

It is found that the induced equations are always the same no matter what prediction variables are introduced. The discovered equations are

$$
\begin{aligned}
& \text { If }\left\langle\text { pKal }>=4.02,<\text { flux }>^{0.1}=1.011+0.289 e^{0.1 *<\text { medium pH }>}\right. \\
& \text { If } \mathrm{D} \_\mathrm{pKa1}=4.03,<\text { flux }>^{0.1}=2.147+0.141 e^{0.1 * \text { medium } \mathrm{pH}>} \\
& \text { If } \mathrm{D} \_\mathrm{pKa1}=4.57,\left\langle\text { flux }>^{0.1}=0.943+0.318 e^{0.1 *<\text { medium } \mathrm{pH}>}\right. \\
& \text { If D_pKa1 }=7.47,<\text { flux }>^{0.1}=3.627-1.376 e^{0.1 *<\text { medium } \mathrm{pH}>} \\
& \text { Prediction error }(e) \quad=0.05868 \\
& \text { Prediction percentage error }(e \%)=3.3651 \% \\
& \text { 1-RE } \\
& =0.9679 \\
& \text { Ratio mean }(r) \\
& =0.99813 \\
& \text { Coefficient of Variation (COV) }=0.04248
\end{aligned}
$$

The induced equations are consistent with the existing domain knowledge. First, they confirm that medium $\mathrm{pH}$ is the most significant variables. Second, they display that medium $\mathrm{pH}$ has a positive effect on acidic drug and a negative effect on basic drugs. That is to say, flux increases with the increase of medium $\mathrm{pH}$ for acidic drugs, while flux decreases with the increase of medium $\mathrm{pH}$ for basic drugs. 
influence is different. The effect is stronger for shallow crack geometry than for deep crack geometry.

It is learned from this application that in addition to the importance of introducing templates based on domain knowledge, it is also important to keep the learning objectives in mind in the learning process.

The application in Section 5.4 studies the dissolution of ionizable drugs using data from chemical engineering. The templates are put into the system based on domain knowledge. The induced equations can predict the $\langle\mathrm{flux}\rangle^{0.1}$ with most of data within $15 \%$ prediction error boundary. In addition, the results are carefully studied by domain engineers. It is found that the results by EDDE are consistent with the existing domain knowledge: 1) medium $\mathrm{pH}$ is the most significant variables; 2) medium $\mathrm{pH}$ has a positive effect on acidic drug and a negative effect on basic drugs. That is to say, flux increases with the increase of medium $\mathrm{pH}$ for acidic drugs, while flux decreases with the increase of medium $\mathrm{pH}$ for basic drugs.

This thesis presents only the final results for this application. In fact, before the final results, there are many iterations of learning. Each time the user studies the feedback information, and decides whether to modify the existing templates, or to add more domain knowledge to the system.

The application in Section 5.5 studies automobile fuel consumption using the data from data repository maintained at University of California, at Irvine. The results show that the more domain knowledge is introduced to the system, the better the results. This can be shown in prediction error and percentage error. The prediction error without the introduction of nonlinear function templates, with the introduction of one nonlinear function template, and with the introduction of two nonlinear function templates are $2.03,1.85$, and 1.82 respectively, while the percentage error are $10.2 \%, 8.93 \%$, and $8.14 \%$ respectively. 


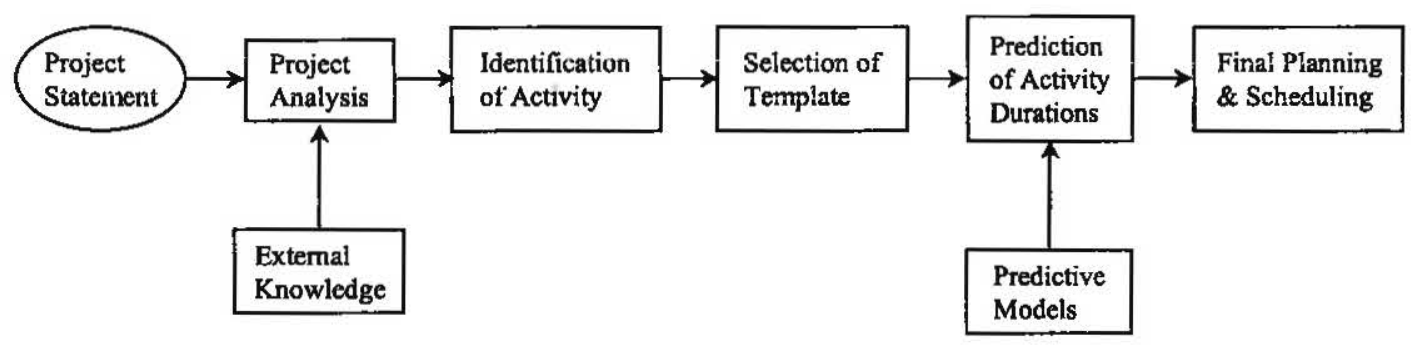

Fig. 5.1 Process of managing a project. 


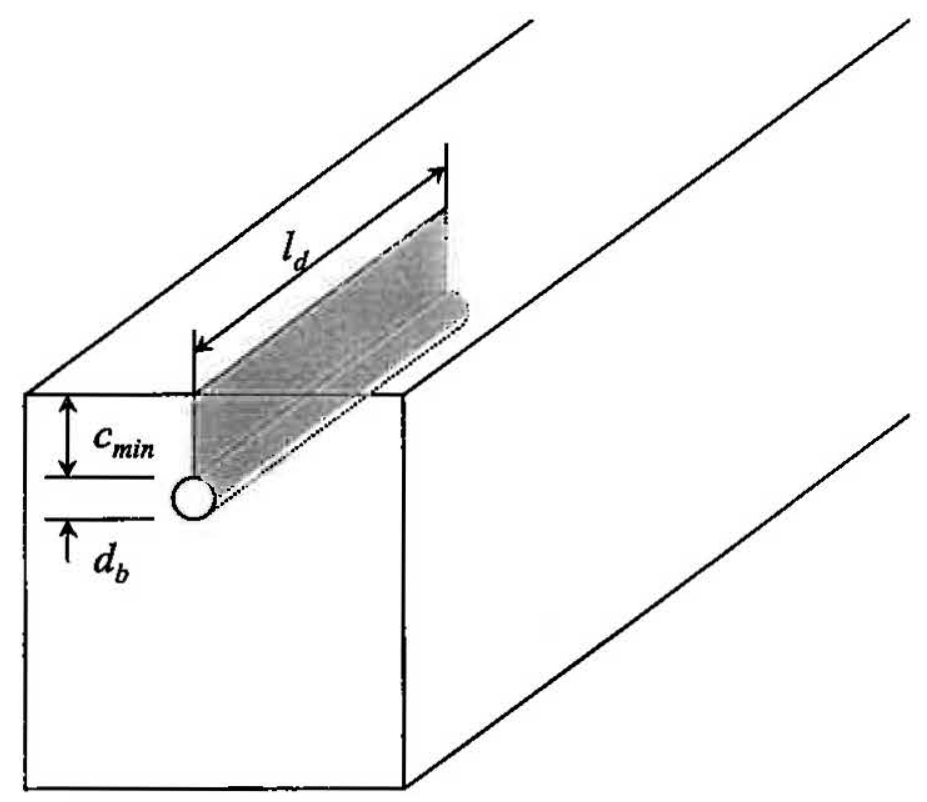

Fig. 5.3 Fracture surface at splice failure. 


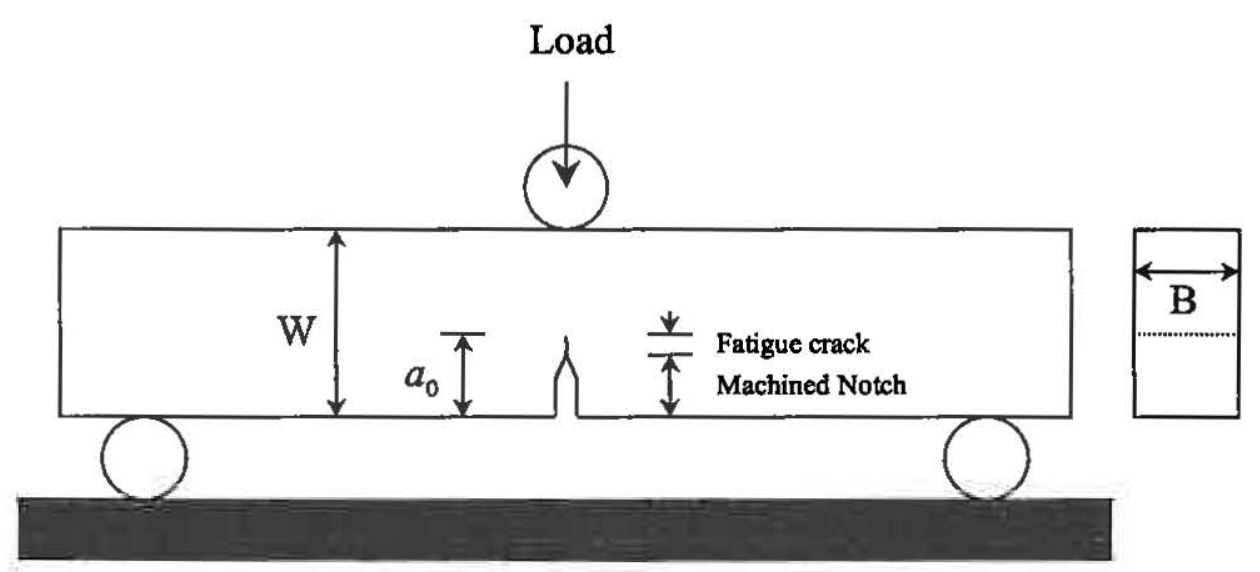

Fig. 5.5 Three point bending test set-up for CTOD. 
assist users with making best decisions.

- The system based on the method of combining machine learning and regression analysis is a monostrategy system, which can solve only certain classes of learning problems as we discussed in Chapters 1 and 3 . To be able to solve more equation discovery problems, more than one learning algorithm would be incorporated into the system [Michalski and Tecuci, 1994].

- The model is measured at two dimensions: prediction strength and comprehensibility. Choosing between alternate models, trade-off parameters are introduced. It would be interesting to use some other methods, such as MDL (Minimum Description Length). The MDL principle [Rissanen, 1989] is a statistical theory that balances model complexity and model error.

- As mentioned in Section 3.1.2, nonlinear functions are necessary for engineering problems. Although EDDE can find nonlinear functions from data, the nonlinear functions are intrinsically linear. It would be interesting to extend the system so that it can find intrinsically nonlinear functions. However, such extension will greatly increase the learning time because the solution can not be found in closed form when the function to be fit is nonlinear in the unknown parameters.

- The system can learn from data with noise, even when the noise variance is $50 \%$ of total variance. However, it is assumed that the noise in data is independent of the variables, and the noise variance is constant in the range of the independent variables (Section 3.3.1). It would be useful to study the performance of EDDE when the noise is related to the independent variable(s), and when the noise variance may change in the range of the independent variables. 
to the data either noise free or with very small noise, the tests show that the system can correctly discover the underlying equations even when the noise variance is $50 \%$ of the total variance.

The system can find the actual equations from the data when the tree interpretation and prediction strength are studied and compared with other methods in Section 4.4. The methods include: regression tree CART, instance based IBL, multivariant linear regression, model tree M5, neural nets, and combinations of these methods.

On the same data set, EDDE generates the equations that divide the data space into two regions as does the known function while CART's equations divides the data space into 13 regions. The results show that EDDE's induced tree summarizes the data more concisely, and its description of the data is more straightforward comparing with CART. Also on the same data set, EDDE generates equations that give the prediction error of 1.1, while other methods give the prediction error from 1.1 to 3.77. On this past set, EDDE outperforms those algorithms, with an improvement rate of up to $260 \%$ in prediction error.

\subsubsection{Application in Different Engineering Domains}

The system EDDE has been applied on actual data sets from different engineering domains. The actual data sets come from civil engineering to study 1) durations of construction activities, 2) development/splice strength of reinforcing bars, and 3) fracture toughness; from chemical engineering to study dissolution of ionizable drugs; and from mechanical engineering to study automobile fuel consumption.

These applications show that the domain knowledge encoded in the algorithm is very general in engineering, so that EDDE can find many different applications in engineering. Each application has its own domain knowledge that is specific to the domain. This domain knowledge is provided to the system in the form of nonlinear 


\subsubsection{Methodology}

The data analysis carried out in engineering is computationally expensive due to its characteristics. A methodology that combines machine learning and regression technique for equation discovery in databases from engineering has been developed in this thesis to overcome the difficulties in data analysis. This method successfully applies heuristics, well studied in machine learning, to solve the problems, which cannot be solved using only traditional regression technique. The data analysis where many variables, nonhomogeneous equations, and unknown significant variables are involved at the same time is successfully solved using the method to intelligently search the domain space to find the equation that satisfy users' requirements.

The important contribution is that the method successfully incorporates general engineering domain knowledge in all aspects of leaming process, from knowledge representation to variable selections to interpretation of the discovered results, and specific domain knowledge in the form of nonlinear function templates when the system is used to solve specific engineering problems. Therefore, the method can be applied in a variety of engineering disciplines.

\subsubsection{Learning Algorithm}

Based on the proposed method, a learning algorithm has been developed and implemented in a computer system EDDE. The contributions embodied in the learning algorithm are listed as follows.

- For the intended learning task, the induced model is expressed as regionequation pairs, which are well represented using a model tree. Unlike previous representations for equation discovery, this representation is able to organize the knowledge into a hierarchy that can be easily understood.

- Equation discovery is a problem of searching the domain space to find the model tree that satisfies users' requirements. Unlike previous systems, the 


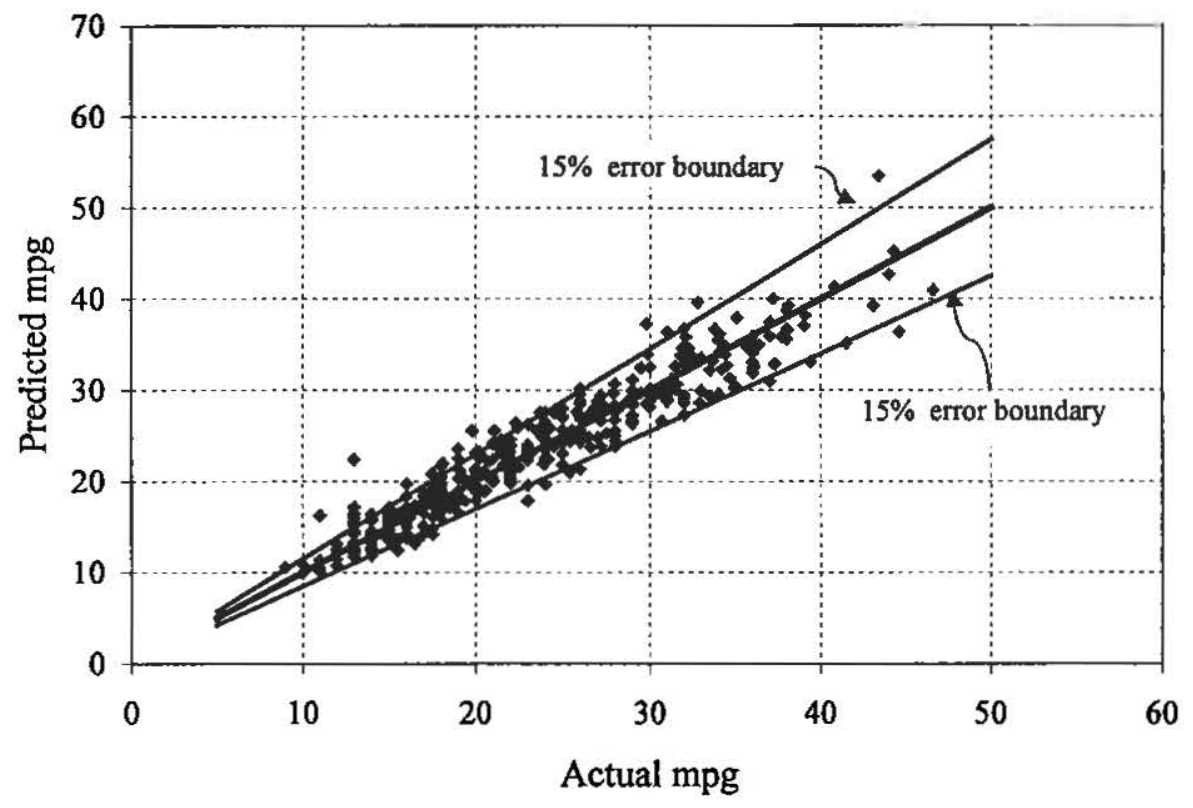

Fig. 5.21 Prediction performance on fuel consumption.

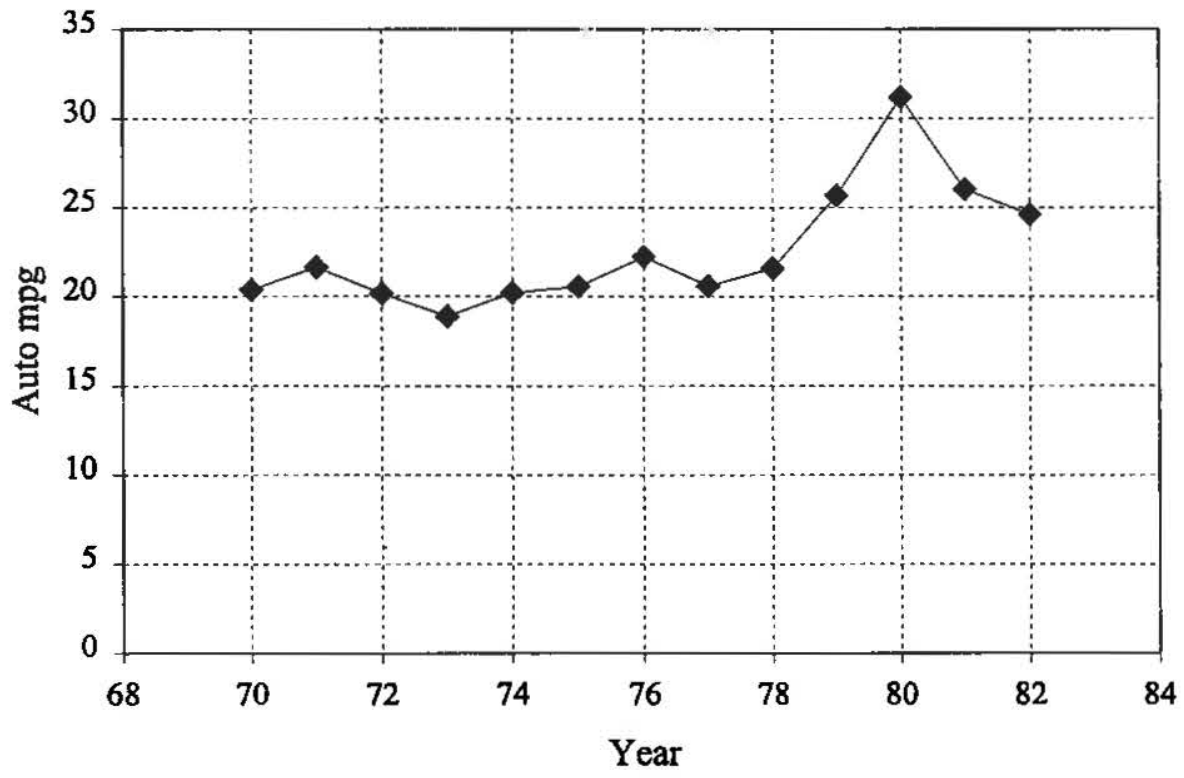

Fig. 5.22 Annual trend of mpg. 


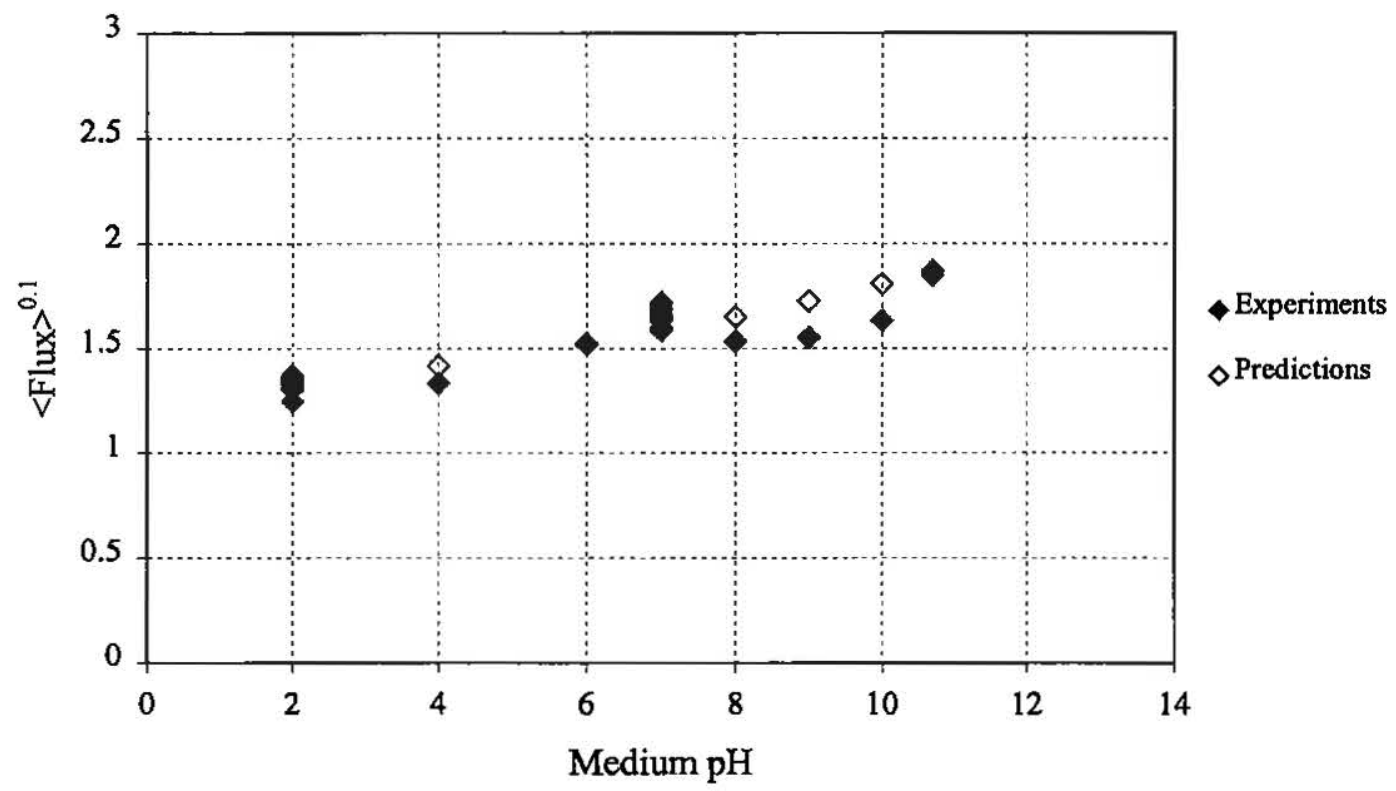

Fig. 5.18 Flux prediction when $\mathrm{pKa}=4.57$.

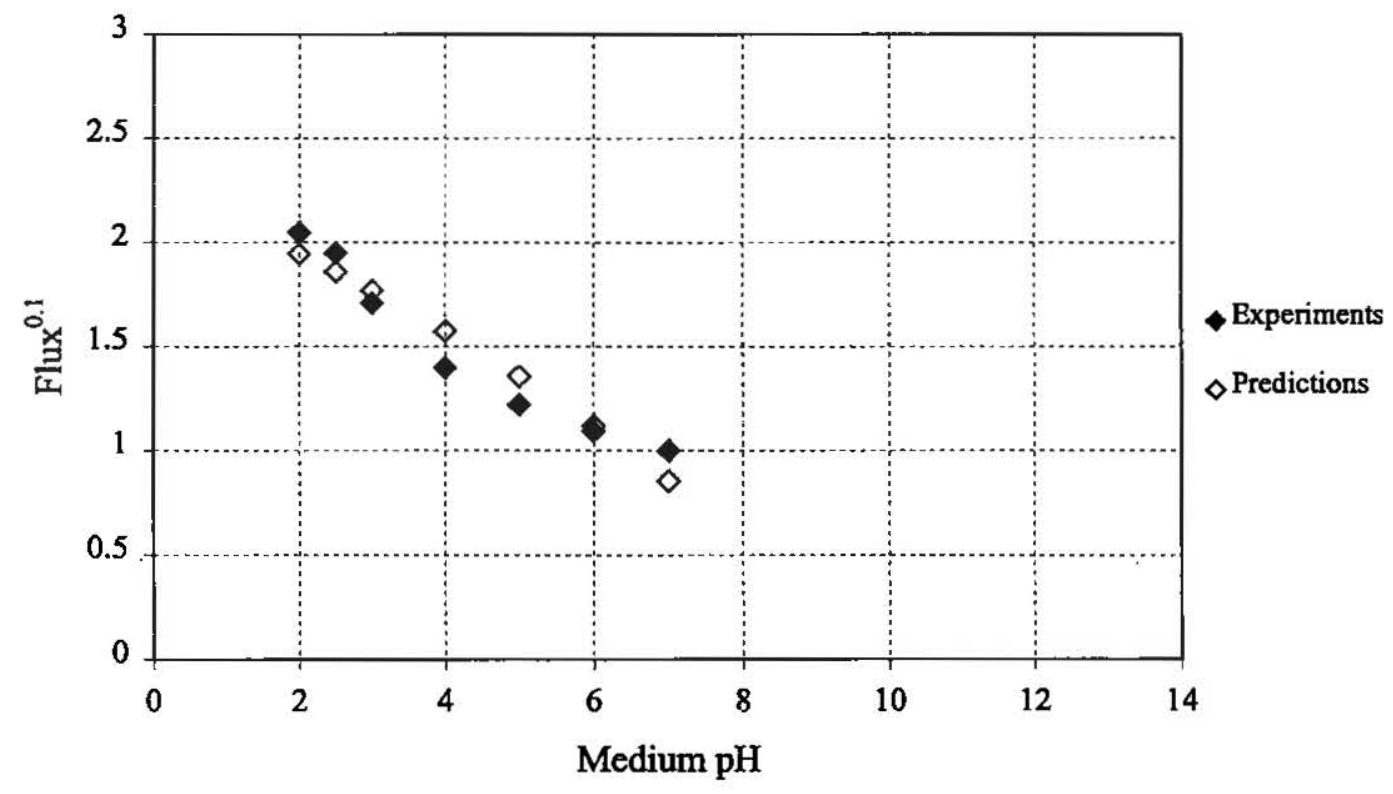

Fig. 5.19 Flux prediction when $\mathrm{pKa}=7.47$. 


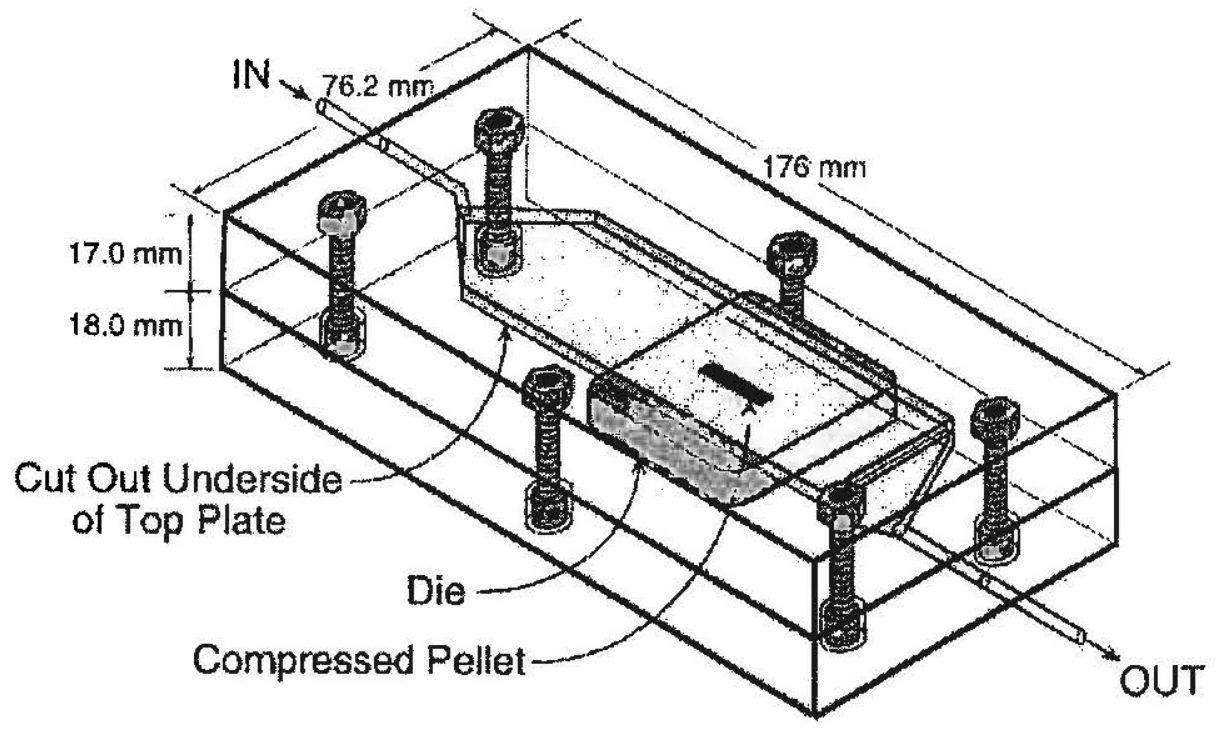

Fig. 5.15 Schematic diagram of the dissolution cell. 


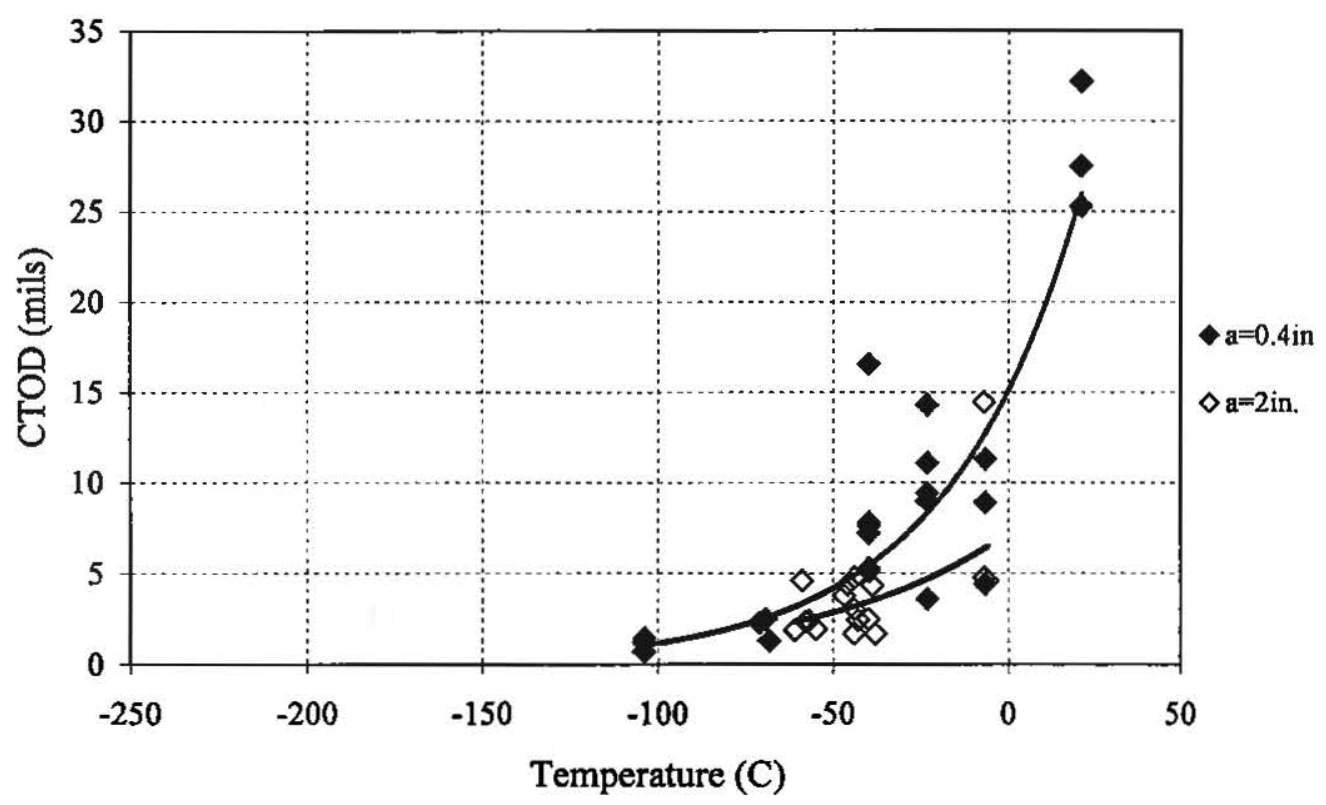

Fig. 5.12 Deep crack geometry

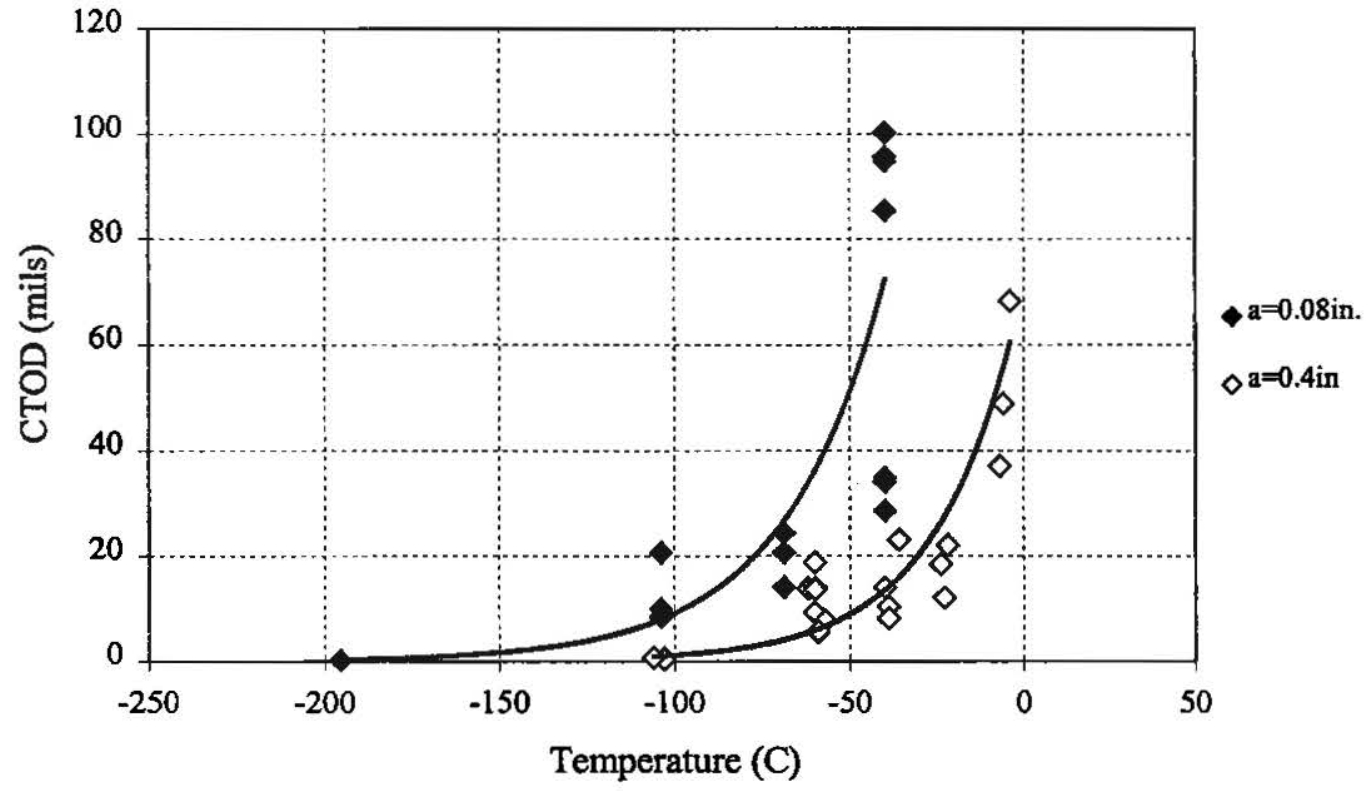

Fig. 5.13 Shallow crack geometry 


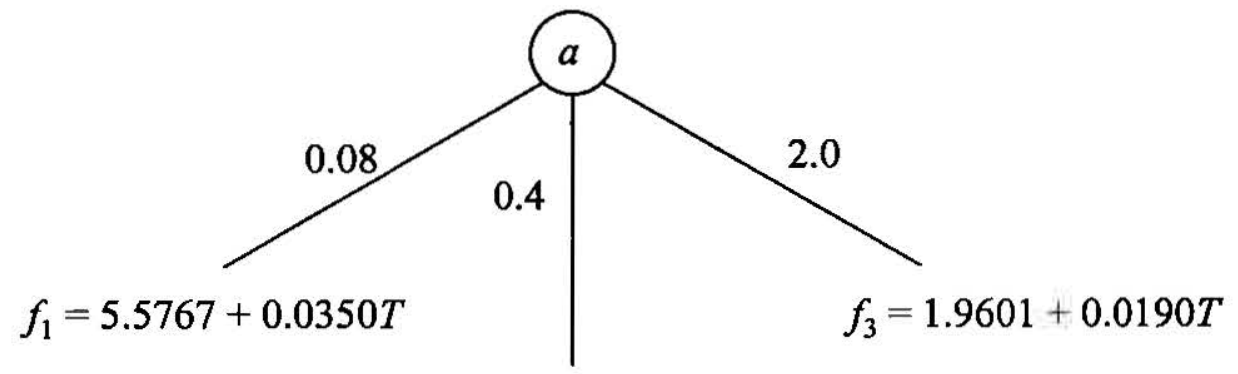

$f_{2}=3.8801+0.0310 T-1.8284 a / \mathrm{W}$

Fig. 5.9 Constant crack depth and varying $a / \mathrm{W}$ ratio

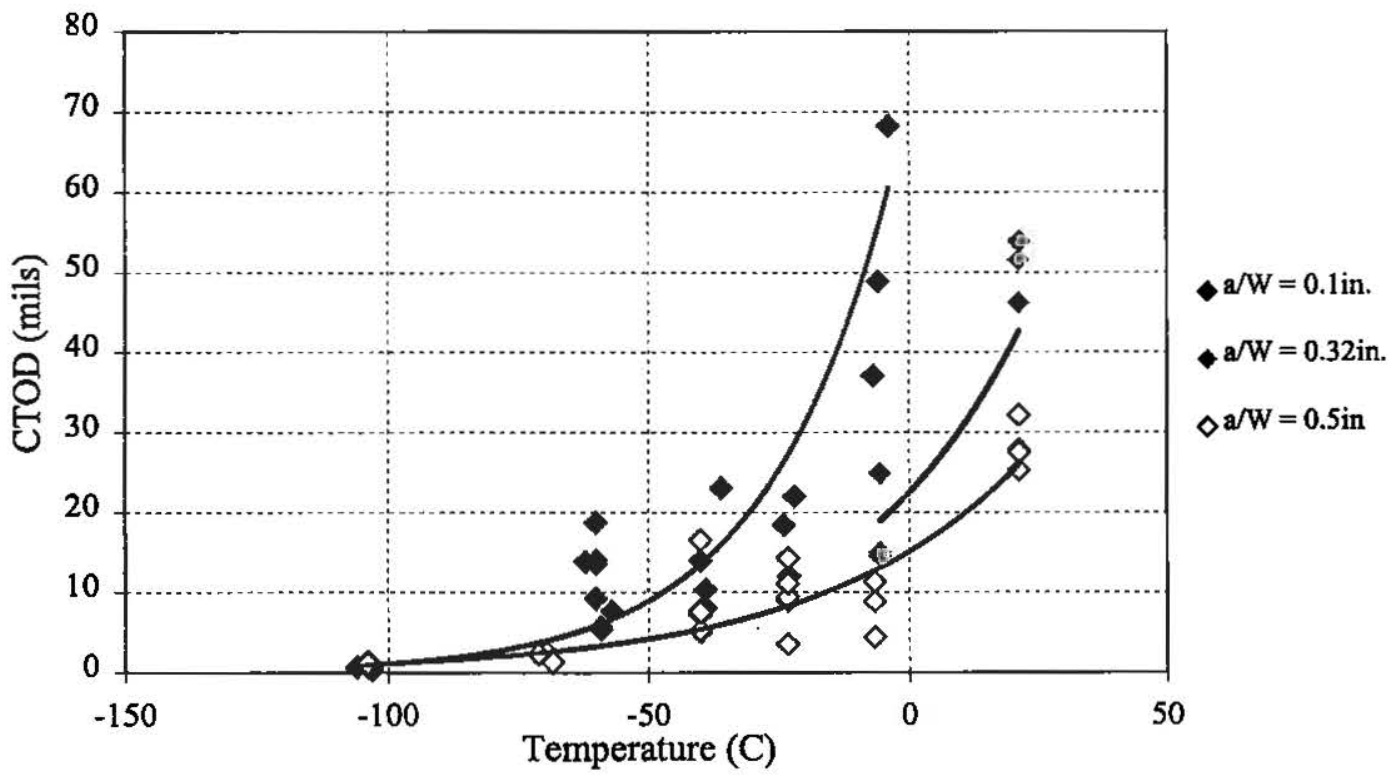

Fig. 5.10 Constant crack depth and varying $a / \mathrm{W}$ ratio 


\subsection{CLOSING REMARKS}

The results shown in this thesis demonstrate the importance of research in assisting engineers in mining useful and meaningful knowledge from data. EDDE is a useful addition to the family of systems for equation discovery in databases. It opens up a paradigm of knowledge discovery research applicable to actual engineering domains. 
Knowledge Discovery in Databases. In Advances in Knowledge Discovery and Data Mining, Eds. Fayyad, U.M., G. Piatetsky-Shapiro, P. Smyth and R. Uthurusamy, AAAI/MIT Press, Cambridge, Mass

Fayyad, U., G. Piatetsky-Shapiro and P. Smyth, (1996b). The KDD Process for Extracting Useful Knowledge from Volumes of Data, Communications of the $A C M$, Nov. 1996, Vol. 39, No. 11.

Forsyth, R. (1989), Machine Learning, Principles and Techniques, Chapter 4, Chapman and Hall Company.

Freeman, J. A. and Skapura, D. M. (1991). Neural Networks: Algorithms, Applications, and Programming Techniques. Addison-Wesley, Reading, MA.

Green, G. H. (1988), The Abacus.2 System for Quantitative Discovery, Master Thesis, Department of Computer Science of the University of Illinois at UrbanaChampaign.

Grzymala-Busse, J. W.(1991), Managing Uncertainty in Expert Systems, Kluwer Academic Publishers.

Hogg, R. V. and J. Ledolter (1987). Engineering Statistics, Macmillan Publishing Company, New York.

Kibler, D., D. W. Aha, and M. K. Albert, (1988), Instance-based Prediction of RealValued Attributes, Technical Report 88-07, ICS, University of California, Irvine.

Kokar, M. M. (1986a), Determining Arguments Of Invariant Functional Descriptions. Machine Learning, 1(4), 1986.

Kokar, M. M. (1986b), Discovering Functional Formulas Through Changing Representation Base, In Proc. of the Fifth National Conference on Artificial Intelligence, 1986.

Langley, P. (1994). Selection of Relevant Features in Machine Learning, Proceedings of the AAAI Fall Symposium on Relevance, New Orleans, LA: AAAI Press.

Langley, P. (1996). Elements of Machine Learning, Morgan Kaufmann Publishers Inc., San Francisco, California.

Langley, P. (1996). Relevance and Insight in Experimental Studies, IEEE Expert, Oct. 1996. 
Proceedings on the Tenth International Conference of Machine Learning, 236-243, University of Massachusetts, Amherst. Morgan Kaufmann.

Quinlan, R. J. (1994), C4.5 : Programs for Machine Learning, Morgan Kaufmann Publisher Inc.

Rao, R. B. (1993), Inverse Engineering: A Machine Learning Approach to Support Engineering Synthesis, Ph. D. dissertation, University of Illinois at UrbanaChampaign.

Rao, R. B. and S. C-Y. Lu (1993), A Knowledge-Based Equation Discovery System for Engineering Domains, IEEE Expert, August, 1993.

Reich, Y. (1991). Building and Improving Design Systems: A Machine Learning Approach, Ph.D. Thesis, Carnegie Mellon University, Pittsburgh, PA.

Reich, Y. and Fenves, S. J. (1989). The Potential of Machine Learning Techniques for Expert Systems. Artificial Intelligence for Engineering Design, Analysis, and Manufacturing, 3(3).

Rissanen, J., (1989). Stochastic Complexity in Statistical Inquiry, World Scientific.

Roddis, W. M. K. and L. Zhang (1997a), Development of Project Activity Duration and Resource Requirement Algorithms Based on Historical Data. Report No, K-TRAN:KU-95-7, Civil and Environmental Engineering Department, University of Kansas.

Roddis, W. M. K. and L. Zhang (1997b), Combination of Machine Learning and Regression Analysis in Knowledge Acquisition, Computing in Civil Engineering.

Schaffer, C. (1990). Domain - Independent Scientific Function Finding, Ph.D. Thesis, Rutgers University.

Schaffer, C. (1991). On Evaluation of Domain - Independent Scientific Function Finding Systems. in Knowledge Discovery in Databases, ed. G. PiatetskyShapiro and W.J. Frawley, The MIT Press.

Schlimmer, J. C. and Fisher, D. (1986), A Case Study of Incremental Concept Induction Science, Proc. of the 5th National Conference on AI, San Mateo, CA, Morgan Kaufmann. 
Engineering, The University of Kansas.

Zytkow, J. M. (1987), Combining many searches in the FAHRENHEIT discovery system. In Proc. of the fourth international workshop on machine learning, San Mateo, California, Morgan Kaufmann.

Zytkow, J. M. and J. Baker (1991), Interactive mining for regularities in databases. In Knowledge Discovery in Databases, Eds. G. Piatetsky-Shapiro and W. J. Frawley, AAAI Press, Menlo Park, California. 


\begin{tabular}{|c|c|c|c|c|c|c|c|c|c|c|c|}
\hline 25 & $\mathbf{Y}$ & $\mathrm{F}$ & $\mathrm{w}$ & $\mathbf{s}$ & $\mathrm{C}$ & 8.968 & 7.064 & 9.401 & 2.232 & 5.746 & 93.117 \\
\hline 26 & $\mathbf{N}$ & $T$ & $\mathrm{w}$ & $\mathrm{T}$ & A & 0.860 & 9.109 & 0.165 & 0.179 & 1.874 & 1.810 \\
\hline 27 & $\mathrm{~N}$ & $T$ & $\mathrm{w}$ & $\mathrm{T}$ & C & 8.321 & 9.327 & 8.544 & 3.387 & 3.416 & 77.922 \\
\hline 28 & $\mathbf{N}$ & $\mathrm{T}$ & $\mathrm{E}$ & $\mathbf{R}$ & B & 6.464 & 1.966 & 5.105 & 9.307 & 7.879 & 47.393 \\
\hline 29 & $\mathbf{N}$ & $\mathrm{F}$ & w & $\mathrm{T}$ & A & 8.614 & 2.309 & 5.619 & 6.981 & 2.297 & 46.450 \\
\hline 30 & $\mathrm{~N}$ & $\mathbf{F}$ & W & $\mathbf{R}$ & C & 7.048 & 0.863 & 0.694 & 1.948 & 1.810 & 14.495 \\
\hline 31 & $\mathrm{Y}$ & $\mathrm{T}$ & $\mathrm{E}$ & $\mathbf{R}$ & A & 5.253 & 7.465 & 1.386 & 8.110 & 6.360 & 120.846 \\
\hline 32 & $\mathrm{~N}$ & $\mathrm{~F}$ & $\mathrm{E}$ & $T$ & B & 2.483 & 7.228 & 0.933 & 6.754 & 6.112 & 12.112 \\
\hline 33 & $\mathrm{~N}$ & $\mathrm{~T}$ & E & $\mathbf{s}$ & A & 3.259 & 7.997 & 6.460 & 6.699 & 1.690 & 57.412 \\
\hline 34 & $\mathrm{Y}$ & $\mathrm{T}$ & w & $s$ & A & 4.770 & 8.370 & 3.376 & 4.781 & 8.929 & 128.694 \\
\hline 35 & $\mathrm{Y}$ & $\mathrm{F}$ & $\mathrm{E}$ & $\mathbf{S}$ & A & 0.724 & 1.963 & 1.453 & 7.967 & 1.174 & 27.387 \\
\hline 36 & $\mathrm{~N}$ & $\mathrm{~T}$ & E & $s$ & B & 0.955 & 8.816 & 8.637 & 8.336 & 0.314 & 73.309 \\
\hline 37 & $Y$ & $T$ & $\mathrm{E}$ & $s$ & A & 8.680 & 5.343 & 6.338 & 9.556 & 4.767 & 125.749 \\
\hline 38 & $\mathrm{~N}$ & $\mathrm{~F}$ & $\mathrm{E}$ & $\mathrm{T}$ & B & 9.803 & 5.312 & 1.381 & 4.961 & 3.711 & 14.306 \\
\hline 39 & $\mathrm{Y}$ & $\mathrm{T}$ & $\mathrm{E}$ & $\mathrm{s}$ & A & 6.339 & 1.922 & 3.249 & 3.975 & 7.408 & 70.450 \\
\hline 40 & $\mathrm{~N}$ & $\mathrm{~F}$ & $\mathrm{E}$ & $\mathrm{s}$ & $\mathrm{C}$ & 6.730 & 5.240 & 2.328 & 8.334 & 0.177 & 28.010 \\
\hline 41 & $\mathrm{Y}$ & $F$ & w & $\mathrm{T}$ & B & 0.991 & 1.090 & 8.700 & 1.203 & 2.220 & 16.870 \\
\hline 42 & $\mathbf{Y}$ & $T$ & w & $\mathrm{s}$ & $\mathrm{C}$ & 2.406 & 3.893 & 8.008 & 4.252 & 9.527 & 59.312 \\
\hline 43 & $\mathrm{Y}$ & $\mathrm{T}$ & $\mathrm{w}$ & $\mathrm{T}$ & A & 0.613 & 5.631 & 8.272 & 0.505 & 5.466 & 64.947 \\
\hline 44 & $\mathbf{Y}$ & $\mathrm{F}$ & $\mathrm{W}$ & $\mathbf{S}$ & B & 7.814 & 1.581 & 9.577 & 2.329 & 5.393 & 46.802 \\
\hline 45 & $\mathrm{~N}$ & $\mathrm{~T}$ & w & $\mathbf{S}$ & $\mathrm{C}$ & 5.180 & 6.756 & 3.260 & 2.674 & 2.417 & 33.926 \\
\hline 46 & $\mathrm{~N}$ & $T$ & $\mathrm{E}$ & $\mathrm{s}$ & A & 2.385 & 0.563 & 8.085 & 4.038 & 7.482 & 65.542 \\
\hline 47 & $\mathbf{N}$ & $\mathrm{T}$ & $\mathbf{w}$ & $\mathbf{S}$ & C & 8.191 & 5.114 & 0.701 & 8.046 & 5.629 & 6.040 \\
\hline 48 & $Y$ & $\mathrm{~F}$ & $\mathrm{w}$ & 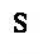 & $\mathrm{C}$ & 1.214 & 6.188 & 2.065 & 9.969 & 9.706 & 47.734 \\
\hline 49 & $\mathrm{Y}$ & $\mathrm{F}$ & E & $\mathrm{s}$ & C & 7.522 & 4.900 & 1.803 & 3.240 & 3.820 & 68.079 \\
\hline 50 & $\mathbf{N}$ & $\mathrm{T}$ & w & $\mathbf{S}$ & B & 6.944 & 7.494 & 5.394 & 6.348 & 4.286 & 48.437 \\
\hline 51 & $\mathbf{N}$ & $\mathbf{F}$ & $\mathrm{E}$ & $\mathbf{R}$ & C & 9.491 & 1.947 & 2.845 & 4.006 & 7.583 & 25.395 \\
\hline 52 & $\mathbf{Y}$ & $\mathrm{T}$ & w & $\mathbf{R}$ & A & 9.053 & 7.561 & 1.854 & 6.157 & 5.084 & 153.166 \\
\hline 53 & $\mathbf{N}$ & $T$ & $\mathrm{E}$ & $\mathbf{R}$ & $\mathbf{A}$ & 8.454 & 3.768 & 6.380 & 8.210 & 3.774 & 58.562 \\
\hline 54 & $\mathbf{Y}$ & $\mathbf{T}$ & w & $\mathbf{s}$ & A & 8.744 & 4.477 & 2.209 & 1.897 & 5.275 & 120.861 \\
\hline 55 & $\mathbf{Y}$ & $\mathbf{T}$ & $\mathbf{w}$ & $\mathbf{R}$ & B & 2.336 & 2.168 & 6.249 & 5.728 & 6.280 & 42.701 \\
\hline 56 & $\mathbf{N}$ & $\mathbf{F}$ & $\mathbf{E}$ & $\mathrm{T}$ & C & 7.949 & 4.964 & 4.558 & 6.531 & 2.142 & 39.508 \\
\hline 57 & $\mathbf{N}$ & $\mathbf{T}$ & w & $\mathbf{S}$ & A & 9.904 & 3.476 & 7.477 & 2.951 & 9.428 & 61.619 \\
\hline 58 & $\mathbf{N}$ & $\mathrm{T}$ & $\mathbf{E}$ & $\mathbf{R}$ & B & 9.509 & 0.152 & 0.489 & 0.732 & 6.612 & 5.634 \\
\hline 59 & $\mathrm{Y}$ & $\mathbf{F}$ & $\mathrm{E}$ & $\mathbf{R}$ & B & 2.800 & 1.337 & 4.340 & 5.652 & 4.869 & 29.336 \\
\hline 60 & $\mathbf{Y}$ & $\mathbf{T}$ & $\mathrm{E}$ & $\mathrm{T}$ & $\mathrm{C}$ & 5.812 & 6.918 & 3.355 & 9.814 & 4.880 & 117.507 \\
\hline 61 & $\mathbf{Y}$ & $F$ & $\mathrm{E}$ & $\mathbf{R}$ & A & 2.478 & 7.360 & 5.273 & 8.579 & 5.399 & 70.566 \\
\hline 62 & $\mathbf{Y}$ & $\mathrm{F}$ & $\mathrm{w}$ & $\mathbf{R}$ & A & 9.803 & 5.818 & 6.545 & 0.957 & 3.674 & 88.831 \\
\hline 63 & $\mathrm{Y}$ & $\mathrm{T}$ & w & $\mathbf{T}$ & B & 6.035 & 5.014 & 6.107 & 2.488 & 0.453 & 104.360 \\
\hline 64 & $\mathrm{~N}$ & $\mathrm{~T}$ & $\mathrm{w}$ & $\mathbf{s}$ & $\mathbf{A}$ & 6.487 & 4.512 & 8.763 & 9.149 & 2.138 & 74.344 \\
\hline
\end{tabular}




\begin{tabular}{|c|c|c|c|c|c|c|c|c|c|c|c|}
\hline 105 & $\mathrm{~N}$ & $\mathbf{F}$ & w & $\mathbf{T}$ & $\mathbf{A}$ & 8.850 & 4.204 & 7.200 & 5.909 & 6.706 & 68.933 \\
\hline 106 & $\mathbf{N}$ & $\mathbf{T}$ & E & $\mathbf{S}$ & B & 4.814 & 0.063 & 7.216 & 5.829 & 9.305 & 63.113 \\
\hline 107 & $\mathbf{Y}$ & $F$ & $\mathrm{E}$ & $T$ & B & 1.974 & 2.638 & 5.847 & 2.004 & 8.314 & 27.549 \\
\hline 108 & $\mathbf{N}$ & $T$ & W & $\mathbf{R}$ & B & 0.846 & 3.646 & 1.264 & 5.552 & 6.352 & 19.865 \\
\hline 109 & $\mathrm{Y}$ & $\mathrm{T}$ & $E$ & $\mathbf{R}$ & B & 1.727 & 1.590 & 9.666 & 0.387 & 4.455 & 39.764 \\
\hline 110 & $\mathrm{Y}$ & $\mathrm{T}$ & W & $\mathrm{T}$ & C & 1.474 & 5.502 & 0.478 & 7.080 & 9.912 & 67.251 \\
\hline 111 & $\mathrm{~N}$ & $\mathrm{~T}$ & $\mathrm{E}$ & $\mathbf{R}$ & A & 7.669 & 4.087 & 1.254 & 8.410 & 6.886 & 19.683 \\
\hline 112 & $\mathrm{Y}$ & $\mathrm{T}$ & $\mathrm{E}$ & $\mathrm{T}$ & B & 0.721 & 3.858 & 0.384 & 7.009 & 5.025 & 44.285 \\
\hline 113 & $\mathrm{Y}$ & $\mathrm{T}$ & W & $\mathbf{S}$ & A & 4.567 & 8.038 & 9.911 & 5.296 & 9.337 & 121.794 \\
\hline 114 & $Y$ & $\mathrm{~F}$ & w & $\mathrm{T}$ & A & 9.217 & 3.116 & 9.892 & 2.745 & 9.316 & 61.570 \\
\hline 115 & $\mathrm{Y}$ & $\mathrm{T}$ & $\mathrm{E}$ & $\mathrm{T}$ & B & 3.494 & 6.377 & 7.073 & 7.170 & 7.733 & 92.248 \\
\hline 116 & $\mathrm{~N}$ & $\mathrm{~T}$ & w & $\mathbf{T}$ & B & 9.864 & 3.455 & 9.748 & 3.880 & 1.239 & 82.064 \\
\hline 117 & $Y$ & $\mathrm{~T}$ & W & $\mathrm{T}$ & B & 4.088 & 8.175 & 4.520 & 4.715 & 3.611 & 115.887 \\
\hline 118 & $\mathbf{Y}$ & $\mathrm{F}$ & w & $\mathbf{R}$ & $\mathrm{C}$ & 8.292 & 8.984 & 2.998 & 2.374 & 9.806 & 101.052 \\
\hline 119 & $\mathbf{Y}$ & $\mathrm{F}$ & W & $\mathbf{S}$ & A & 0.027 & 9.413 & 3.035 & 6.910 & 3.814 & 72.240 \\
\hline 120 & $\mathrm{~N}$ & $\mathrm{~F}$ & $\mathrm{E}$ & $\mathbf{S}$ & $\mathbf{A}$ & 0.452 & 3.622 & 8.897 & 3.043 & 7.283 & 75.330 \\
\hline 121 & $\mathbf{Y}$ & $\mathrm{F}$ & W & $\mathbf{S}$ & C & 8.950 & 9.037 & 4.831 & 9.036 & 7.951 & 101.656 \\
\hline 122 & $\mathrm{Y}$ & $F$ & $\mathrm{E}$ & $\mathbf{S}$ & $\mathrm{C}$ & 2.794 & 1.270 & 3.030 & 6.250 & 5.554 & 28.598 \\
\hline 123 & $\mathrm{Y}$ & $F$ & E & $\mathrm{T}$ & $\mathrm{C}$ & 2.706 & 1.462 & 3.548 & 2.602 & 2.027 & 29.244 \\
\hline 124 & $\mathbf{N}$ & $\mathrm{T}$ & E & $\mathrm{s}$ & $\mathrm{C}$ & 8.665 & 3.550 & 4.474 & 7.848 & 0.379 & 39.369 \\
\hline 125 & $\mathbf{N}$ & $\mathrm{T}$ & E & $s$ & C & 1.724 & 3.446 & 2.754 & 8.560 & 4.682 & 25.100 \\
\hline 126 & $\mathbf{N}$ & $\mathbf{T}$ & E & $\mathbf{T}$ & A & 2.054 & 1.747 & 1.677 & 2.219 & 9.211 & 15.292 \\
\hline 127 & $\mathbf{Y}$ & $T$ & E & $\mathbf{R}$ & C & 9.675 & 6.160 & 2.593 & 8.221 & 2.035 & 139.656 \\
\hline 128 & $\mathbf{N}$ & $\mathrm{T}$ & E & $\mathrm{S}$ & B & 5.802 & 3.857 & 4.949 & 5.677 & 7.085 & 42.027 \\
\hline 129 & $\mathbf{N}$ & $T$ & $\mathrm{E}$ & $\mathrm{T}$ & C & 9.135 & 6.047 & 5.554 & 7.908 & 6.004 & 47.257 \\
\hline 130 & $\mathbf{N}$ & $\mathrm{T}$ & w & $\mathbf{R}$ & B & 9.841 & 3.770 & 2.407 & 8.712 & 1.611 & 23.397 \\
\hline 131 & $\mathbf{N}$ & $T$ & E & $\mathrm{T}$ & B & 1.333 & 3.426 & 7.062 & 6.627 & 6.071 & 63.552 \\
\hline 132 & $\mathbf{N}$ & $\mathbf{F}$ & $\mathbf{E}$ & $s$ & B & 5.694 & 7.863 & 9.769 & 8.950 & 7.579 & 81.756 \\
\hline 133 & $\mathbf{Y}$ & $T$ & $\mathbf{w}$ & $\mathbf{R}$ & $\mathbf{B}$ & 9.256 & 0.186 & 3.366 & 4.972 & 3.989 & 80.169 \\
\hline 134 & $\mathbf{N}$ & $\mathbf{F}$ & W & $\mathbf{S}$ & $\mathbf{A}$ & 7.551 & 3.376 & 0.505 & 5.726 & 4.506 & 8.893 \\
\hline 135 & $\mathbf{Y}$ & $\mathbf{F}$ & w & $\mathrm{T}$ & C & 3.042 & 8.275 & 5.070 & 0.958 & 6.528 & 77.830 \\
\hline 136 & $\mathbf{Y}$ & $\mathrm{T}$ & W & $\mathbf{S}$ & $\mathbf{A}$ & 8.913 & 4.866 & 3.784 & 0.296 & 8.481 & 123.951 \\
\hline 137 & $\mathbf{Y}$ & $\mathbf{T}$ & $\mathbf{w}$ & $\mathbf{R}$ & B & 3.880 & 7.273 & 4.802 & 5.193 & 4.557 & 108.489 \\
\hline 138 & $\mathrm{~N}$ & $\Upsilon$ & $\mathrm{E}$ & $\mathbf{R}$ & $\mathbf{A}$ & 5.497 & 8.355 & 0.255 & 9.448 & 4.179 & 9.947 \\
\hline 139 & $\mathbf{N}$ & $\mathrm{F}$ & W & $\mathbf{R}$ & $\mathbf{A}$ & 6.423 & 5.140 & 1.694 & 6.907 & 3.318 & 11.799 \\
\hline 140 & $\mathbf{N}$ & $\mathbf{T}$ & $\mathrm{E}$ & $\mathrm{S}$ & B & 4.101 & 0.292 & 3.695 & 6.820 & 7.781 & 37.036 \\
\hline 141 & $\mathbf{N}$ & $\mathrm{T}$ & E & $\mathbf{S}$ & $\mathrm{C}$ & 9.259 & 5.611 & 3.724 & 7.652 & 6.329 & 38.404 \\
\hline 142 & $\mathbf{Y}$ & $\mathbf{F}$ & W & $\mathbf{S}$ & B & 5.845 & 7.228 & 6.373 & 8.325 & 5.132 & 78.609 \\
\hline 143 & $\mathbf{N}$ & $\mathbf{F}$ & W & $\mathbf{R}$ & C & 9.325 & 3.777 & 9.723 & 2.817 & 0.802 & 79.254 \\
\hline 144 & $\mathbf{Y}$ & $\mathbf{F}$ & $\mathbf{E}$ & $\mathbf{R}$ & C & 0.007 & 3.987 & 4.397 & 7.250 & 2.129 & 35.541 \\
\hline
\end{tabular}




\begin{tabular}{llllllllllll}
185 & Y & T & E & R & A & 4.370 & 7.371 & 7.682 & 4.120 & 4.641 & 111.314 \\
186 & Y & T & E & S & B & 2.123 & 4.663 & 6.802 & 3.481 & 2.969 & 67.981 \\
187 & N & T & E & T & A & 8.019 & 8.450 & 3.974 & 2.286 & 6.233 & 36.318 \\
188 & N & T & W & T & B & 9.020 & 4.457 & 8.800 & 8.078 & 6.905 & 76.510 \\
189 & N & T & W & R & A & 3.748 & 2.529 & 8.446 & 9.191 & 2.445 & 70.718 \\
190 & N & F & W & S & B & 7.279 & 0.048 & 3.629 & 8.237 & 4.748 & 34.100 \\
191 & N & F & W & R & A & 9.498 & 3.510 & 5.847 & 4.953 & 3.516 & 53.709 \\
192 & Y & F & E & R & C & 4.806 & 4.834 & 1.919 & 1.247 & 0.535 & 61.573 \\
193 & Y & T & W & R & C & 9.440 & 1.390 & 7.541 & 1.884 & 6.334 & 94.834 \\
194 & Y & F & E & S & B & 9.687 & 4.748 & 6.639 & 2.315 & 3.361 & 76.547 \\
195 & Y & T & W & T & B & 7.411 & 2.653 & 8.991 & 7.040 & 3.118 & 86.362 \\
196 & N & F & W & T & C & 4.650 & 4.024 & 5.052 & 1.666 & 2.496 & 45.938 \\
197 & Y & F & E & T & A & 4.735 & 7.731 & 7.089 & 5.023 & 4.293 & 79.484 \\
198 & N & F & W & R & C & 5.499 & 6.732 & 3.292 & 6.911 & 9.405 & 22.076 \\
199 & Y & F & W & R & B & 0.215 & 6.926 & 6.952 & 9.052 & 3.102 & 56.424 \\
200 & N & T & W & S & C & 7.963 & 8.160 & 5.720 & 1.991 & 6.197 & 53.406 \\
\hline
\end{tabular}

\section{A.2 TESTING DATA}

\begin{tabular}{cccccccccccc}
\hline index & $x_{1}$ & $x_{2}$ & $x_{3}$ & $x_{4}$ & $x_{5}$ & $x_{6}$ & $x_{7}$ & $x_{8}$ & $x_{9}$ & $x_{10}$ & $y$ \\
\hline 5 & Y & F & E & T & C & 3.760 & 1.730 & 3.550 & 5.410 & 3.240 & 32.720 \\
13 & N & F & W & S & A & 4.470 & 6.160 & 1.430 & 6.620 & 2.100 & 18.240 \\
15 & N & F & W & T & B & 8.710 & 3.200 & 2.300 & 0.860 & 7.490 & 20.790 \\
16 & N & F & W & T & C & 0.870 & 4.240 & 5.730 & 7.780 & 8.190 & 45.420 \\
17 & Y & T & E & S & B & 4.600 & 6.590 & 5.760 & 9.880 & 5.510 & 106.770 \\
28 & N & T & E & R & B & 6.460 & 1.970 & 5.110 & 9.310 & 7.880 & 47.390 \\
40 & N & F & E & S & C & 6.730 & 5.240 & 2.330 & 8.330 & 0.180 & 28.010 \\
42 & Y & T & W & S & C & 2.410 & 3.890 & 8.010 & 4.250 & 9.530 & 59.310 \\
43 & Y & T & W & T & A & 0.610 & 5.630 & 8.270 & 0.510 & 5.470 & 64.950 \\
48 & Y & F & W & S & C & 1.210 & 6.190 & 2.060 & 9.970 & 9.710 & 47.730 \\
52 & Y & T & W & R & A & 9.050 & 7.560 & 1.850 & 6.160 & 5.080 & 153.170 \\
53 & N & T & E & R & A & 8.450 & 3.770 & 6.380 & 8.210 & 3.770 & 58.560 \\
57 & N & T & W & S & A & 9.900 & 3.480 & 7.480 & 2.950 & 9.430 & 61.620 \\
64 & N & T & W & S & A & 6.490 & 4.510 & 8.760 & 9.150 & 2.140 & 74.340 \\
65 & N & T & W & T & C & 5.720 & 0.490 & 4.480 & 4.910 & 8.210 & 35.070 \\
67 & N & F & W & S & B & 2.610 & 3.800 & 1.280 & 2.240 & 5.620 & 15.010 \\
80 & Y & T & E & R & C & 8.880 & 6.060 & 7.920 & 9.730 & 2.120 & 138.570 \\
& & & & & & & 161 & & & &
\end{tabular}




\section{APPENDIX B}

\section{THE DATA SET USED IN SECTION 5.2}

\section{B.1 THE INPUT DATA SET}

\begin{tabular}{|c|c|c|c|c|c|c|c|c|c|c|c|c|c|c|}
\hline Test No. & $\mathrm{n}$ & $\begin{array}{c}\mathrm{l}_{\mathrm{d}} \\
\text { (in.) }\end{array}$ & $\begin{array}{c}\mathrm{d}_{\mathrm{b}} \\
\text { (in.) }\end{array}$ & $\begin{array}{c}\mathrm{c}_{\mathrm{so}} \\
\text { (in.) }\end{array}$ & $\begin{array}{l}\mathrm{c}_{\mathrm{si}} \\
\text { (in.) }\end{array}$ & $\begin{array}{c}c_{b} \\
\text { (in.) }\end{array}$ & $\begin{array}{c}\mathrm{b} \\
\text { (in.) }\end{array}$ & $\begin{array}{c}\mathrm{h} \\
\text { (in.) }\end{array}$ & $\begin{array}{c}\mathrm{d} \\
\text { (in.) }\end{array}$ & $\begin{array}{c}\mathrm{f}_{\mathrm{c}} \\
\text { (psi) }\end{array}$ & $\begin{array}{c}f_{y} \\
\text { (ksi) }\end{array}$ & $\begin{array}{c}\mathrm{f}_{\mathrm{s}} * \\
(\mathrm{ksi})\end{array}$ & $\begin{array}{c}\mathrm{T}_{c} / \mathrm{f}_{c}{ }^{1 / 2} \\
\left(\text { in. }^{2}\right)\end{array}$ & $\begin{array}{l}T_{d} / f_{e}^{1 / 4} \\
\left(\text { in. }{ }^{2}\right)\end{array}$ \\
\hline \multicolumn{15}{|c|}{ Chinn(1956) } \\
\hline D31 & 1 & 5.50 & 0.375 & 1.470 & & 0.830 & 3.69 & - & - & 4700 & 79.00 & 60.70 & 98 & 810 \\
\hline D36 & 1 & 5.50 & 0.375 & 1.470 & & 0.560 & 3.69 & . & - & 4410 & 79.00 & 49.21 & 82 & 667 \\
\hline D10 & 1 & 7.00 & 0.750 & 1.060 & & 1.480 & 3.62 & . & - & 4370 & 57.00 & 26.41 & 176 & 1429 \\
\hline D20 & 1 & 7.00 & 0.750 & 1.125 & & 1.420 & 3.75 & . & - & 4230 & 57.00 & 27.12 & 183 & 1479 \\
\hline D22 & 1 & 7.00 & 0.750 & 1.095 & & 0.800 & 3.69 & , & . & 4480 & 57.00 & 23.97 & 158 & 1289 \\
\hline D13 & 1 & 11.00 & 0.750 & 2.905 & & 1.440 & 7.31 & - & - & 4820 & 57.00 & 49.14 & 311 & 2595 \\
\hline D14 & 1 & 11.00 & 0.750 & 1.095 & & 0.830 & 3.69 & - & - & 4820 & 57.00 & 32.82 & 208 & 1733 \\
\hline D15 & 1 & 11.00 & 0.750 & 2.875 & & 0.620 & 7.25 & $=$ & $=$ & 4290 & 57.00 & 42.45 & 285 & 2308 \\
\hline D21 & 1 & 11.00 & 0.750 & 2.905 & & 1.470 & 7.31 & - & - & 4480 & 57.00 & 43.53 & 286 & 2341 \\
\hline D29 & 1 & 11.00 & 0.750 & 1.095 & & 1.390 & 3.69 & - & - & 7480 & 57.00 & 44.62 & 227 & 2111 \\
\hline D3 & 2 & 11.00 & 0.750 & 1.500 & 0.500 & 1.500 & 9.00 & . & $=$ & 4350 & 57.00 & 37.15 & 248 & 2013 \\
\hline D32 & 1 & 11.00 & 0.750 & 2.875 & & 1.470 & 7.25 & - & - & 4700 & 57.00 & 46.24 & 297 & 2457 \\
\hline D38 & 1 & 11.00 & 0.750 & 1.560 & & 1.520 & 4.62 & . & - & 3160 & 57.00 & 28.50 & 223 & 1673 \\
\hline D39 & 1 & 11.00 & 0.750 & 1.095 & & 1.560 & 3.69 & . & - & 3160 & 57.00 & 28.05 & 220 & 1646 \\
\hline D5 & 1 & 11.00 & 0.750 & 2.000 & & 1.500 & 5.50 & . & - & 4180 & 57.00 & 44.76 & 305 & 2449 \\
\hline D6 & 2 & 11.00 & 0.750 & 1.500 & 0.625 & 1.160 & 7.25 & . & $=$ & 4340 & 57.00 & 33.48 & 224 & 1815 \\
\hline D7 & 1 & 11.00 & 0.750 & 1.060 & & 1.270 & 3.62 & 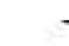 & - & 4450 & 57.00 & 34.15 & 225 & 1840 \\
\hline D8 & 2 & 11.00 & 0.750 & 1.500 & 0.625 & 1.480 & 7.25 & . & . & 4570 & 57.00 & 36.28 & 236 & 1942 \\
\hline D9 & 1 & 11.00 & 0.750 & 1.060 & & 1.440 & 3.62 & - & - & 4380 & 57.00 & 35.33 & 235 & 1911 \\
\hline D34 & 1 & 12.50 & 0.750 & 1.060 & & 1.490 & 3.62 & 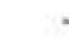 & - & 3800 & 57.00 & 37.46 & 267 & 2099 \\
\hline D12 & 1 & 16.00 & 0.750 & 1.125 & & 1.620 & 3.75 & 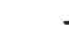 & . & 4530 & 57.00 & 46.37 & 303 & 2487 \\
\hline D17 & 1 & 16.00 & 0.750 & 1.095 & & 0.800 & 3.69 & . & - & 3580 & 57.00 & 40.56 & 298 & 2307 \\
\hline D19** & 1 & 16.00 & 0.750 & 2.905 & & 1.700 & 7.31 & 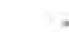 & - & 4230 & 57.00 & 57.60 & 390 & 3142 \\
\hline D23 & 1 & 16.00 & 0.750 & 1.060 & & 0.780 & 3.62 & 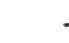 & - & 4450 & 57.00 & 39.70 & 262 & 2139 \\
\hline
\end{tabular}




\begin{tabular}{|c|c|c|c|c|c|c|c|c|c|c|c|c|c|c|}
\hline $11 \mathrm{~F} 36 \mathrm{a}$ & 2 & 49.50 & 1.410 & 4.590 & 4.635 & 1.500 & 24.09 & 18.00 & 15.79 & 4570 & 73.00 & 64.66 & 1492 & 12268 \\
\hline $11 \mathrm{~F} 36 \mathrm{~b}$ & 2 & 49.50 & 1.410 & 4.590 & 4.605 & 1.470 & 24.03 & 18.00 & 15.83 & 3350 & 65.00 & 60.09 & 1620 & 12321 \\
\hline $11 F 42 a$ & 2 & 57.75 & 1.410 & 4.590 & 4.590 & 1.480 & 24.00 & 18.00 & 15.82 & 3530 & 65.00 & 64.57 & 1695 & 13067 \\
\hline 11 F48a** & 2 & 66.00 & 1.410 & 4.590 & 4.620 & 1.530 & 24.16 & 18.03 & 15.80 & 3140 & 73.00 & 73.91 & 2058 & 15402 \\
\hline $11 \mathrm{~F} 48 \mathrm{~b} * *$ & 2 & 66.00 & 1.410 & 4.590 & 4.665 & 1.580 & 24.15 & 18.22 & 15.93 & 3330 & 65.00 & 72.24 & 1953 & 14835 \\
\hline $11 \mathrm{R} 48 \mathrm{a}$ & 2 & 66.00 & 1.410 & 4.590 & 4.670 & 1.500 & 24.16 & 18.03 & 15.83 & 5620 & 93.00 & 82.81 & 1723 & 14920 \\
\hline $11 \mathrm{R} 48 \mathrm{~b}$ & 2 & 66.00 & 1.410 & 4.590 & 4.700 & 2.060 & 24.22 & 18.19 & 15.43 & 3100 & 93.00 & 73.20 & 2051 & 15303 \\
\hline $11 \mathrm{~F} 60 \mathrm{a}^{* *}$ & 2 & 82.50 & 1.410 & 4.590 & 4.575 & 1.590 & 23.97 & 18.09 & 15.83 & 2610 & 73.00 & 84.80 & 2589 & 18508 \\
\hline $11 \mathrm{~F} 60 \mathrm{~b}^{* *}$ & 2 & 82.50 & 1.410 & 4.590 & 4.590 & 1.500 & 24.00 & 18.09 & 15.92 & 4090 & 65.00 & 78.02 & 1903 & 15219 \\
\hline $11 \mathrm{R} 60 \mathrm{a}$ & 2 & 82.50 & 1.410 & 4.590 & 4.590 & 1.410 & 24.00 & 18.12 & 16.01 & 2690 & 93.00 & 77.19 & 2322 & 16720 \\
\hline $11 \mathrm{R} 60 \mathrm{~b}$ & 2 & 82.50 & 1.410 & 4.590 & 4.575 & 1.750 & 24.00 & 18.03 & 15.58 & 3460 & 93.00 & 90.35 & 2396 & 18378 \\
\hline \multicolumn{15}{|c|}{ Thompson_etal(1975) } \\
\hline $6-12-4 / 2 / 2-6 / 6$ & 6 & 12.00 & 0.750 & 2.000 & 2.000 & 2.000 & 33.00 & 13.00 & 10.63 & 3730 & 61.70 & 57.96 & 418 & 3263 \\
\hline $8-18-4 / 3 / 2-6 / 6$ & 6 & 18.00 & 1.000 & 2.000 & 2.000 & 3.000 & 36.00 & 13.00 & 9.50 & 4710 & 59.30 & 57.00 & 656 & 5435 \\
\hline $8-18-4 / 3 / 2.5-4 / 6$ & 6 & 18.00 & 1.000 & 2.500 & 2.000 & 3.000 & 36.00 & 13.00 & 9.50 & 2920 & 59.30 & 50.86 & 744 & 5466 \\
\hline $8-24-4 / 2 / 2-6 / 6$ & 6 & 24.00 & 1.000 & 2.000 & 2.000 & 2.000 & 36.00 & 13.00 & 10.50 & 3105 & 59.30 & 51.89 & 736 & 5491 \\
\hline $11-25-6 / 2 / 3-5 / 5$ & 5 & 25.00 & 1.410 & 3.000 & 3.000 & 2.000 & 44.06 & 13.01 & 10.30 & 3920 & 66.30 & 45.00 & 1121 & 8873 \\
\hline $11-30-4 / 2 / 2-6 / 6$ & 6 & 30.00 & 1.410 & 2.000 & 2.000 & 2.000 & 40.88 & 13.01 & 10.30 & 2865 & 60.50 & 39.56 & 1153 & 8436 \\
\hline $11-30-4 / 2 / 4-6 / 6$ & 6 & 30.00 & 1.410 & 4.000 & 2.000 & 2.000 & 44.88 & 13.01 & 10.30 & 3350 & 63.40 & 45.90 & 1237 & 9413 \\
\hline $11-30-4 / 2 / 2.7-4 / 6$ & 4 & 30.00 & 1.410 & 2.700 & 2.000 & 2.000 & 44.88 & 13.01 & 10.30 & 4420 & 63.30 & 58.48 & 1372 & 11189 \\
\hline $11-45-4 / 1 / 2-6 / 6$ & 6 & 45.00 & 1.410 & 2.000 & 2.000 & 1.000 & 40.88 & 13.01 & 11.30 & 3520 & 60.50 & 46.72 & 1228 & 9462 \\
\hline $14-60-4 / 2 / 2-5 / 5$ & 5 & 60.00 & 1.693 & 2.000 & 2.000 & 2.000 & 37.50 & 16.15 & 13.30 & 2865 & 57.70 & 48.13 & 2023 & 14801 \\
\hline $14-60-4 / 2 / 4-5 / 5$ & 5 & 60.00 & 1.693 & 4.000 & 2.000 & 2.000 & 41.50 & 16.00 & 13.15 & 3200 & 57.70 & 56.64 & 2253 & 16944 \\
\hline \multicolumn{15}{|l|}{ Zekany(1981) } \\
\hline $9-53-B-N$ & 5 & 16.00 & 1.128 & 2.000 & 1.423 . & 2.000 & 27.25 & 16.00 & 13.44 & 5650 & 62.80 & 47.77 & 636 & 5510 \\
\hline $\mathrm{N}-\mathrm{N}-80 \mathrm{~B}$ & 4 & 22.00 & 1.410 & 2.000 & 1.849 & 2.000 & 27.25 & 16.01 & 13.30 & 3825 & 60.10 & 38.53 & 972 & 7642 \\
\hline \multicolumn{15}{|c|}{ Choi_etal(1990\&1991) } \\
\hline 1.1 & 2 & 12.00 & 0.625 & 2.000 & 2.000 & 1.000 & 10.50 & 16.00 & 14.69 & 5360 & 63.80 & 61.51 & 260 & 2229 \\
\hline $1.2^{* *}$ & 3 & 12.00 & 0.625 & 2.000 & 2.000 & 1.000 & 15.75 & 16.00 & 14.69 & 5360 & 63.80 & 64.00 & 271 & 319 \\
\hline 2.3 & 2 & 12.00 & 0.750 & 2.000 & 2.000 & 1.000 & 11.00 & 16.01 & 14.63 & 6010 & 70.90 & 51.34 & 291 & 2566 \\
\hline 2.1 & 2 & 12.00 & 0.750 & 2.000 & 2.000 & 1.000 & 11.00 & 16.01 & 14.63 & 6010 & 63.80 & 45.67 & 259 & 2282 \\
\hline 3.3 & 2 & 16.00 & 1.000 & 2.000 & 2.000 & 1.500 & 12.00 & 16.00 & 14.00 & 5980 & 63.80 & .00 & 39 & 363 \\
\hline 3.1 & 2 & 16.00 & 1.000 & 2.000 & 2.000 & 1.500 & 12.00 & 14.00 & 12.00 & 5980 & 67.00 & 42.81 & 437 & 3846 \\
\hline 4.3 & 2 & 24.00 & 1.410 & 2.000 & 2.000 & 2.000 & 13.65 & 16.01 & 13.30 & 5850 & 63.10 & 37.93 & 774 & 6765 \\
\hline 4.1 & 2 & 24.00 & 1.410 & 2.000 & 2.000 & 2.000 & 13.65 & 16.01 & 13.30 & 5850 & 64.60 & 40.37 & 823 & 7201 \\
\hline \multicolumn{15}{|c|}{ Hester_etal(1991\&1993) } \\
\hline 1.1 & 3 & 16.00 & 1.000 & 2.000 & 1.500 & 2.000 & 16.00 & 16.00 & 13.50 & 5990 & 63.80 & 50.13 & 512 & 4501 \\
\hline 2.1 & 3 & 16.00 & 1.000 & 2.000 & 1.500 & 1.840 & 16.00 & 16.33 & 13.99 & 6200 & 69.00 & 46.25 & 464 & 4118 \\
\hline 3.1 & 3 & 16.00 & 1.000 & 2.000 & 1.500 & 2.040 & 16.09 & 16.23 & 13.69 & 6020 & 71.10 & 46.86 & 477 & 4202 \\
\hline 4.1 & 3 & 16.00 & 1.000 & 2.000 & 1.500 & 2.100 & 16.08 & 16.22 & 13.62 & 6450 & 71.10 & 42.36 & 417 & 3734 \\
\hline 5.1 & 3 & 16.00 & 1.000 & 2.000 & 1.500 & 2.050 & 16.09 & 16.27 & 13.72 & 5490 & 69.00 & 39.86 & 425 & 3658 \\
\hline 6.1 & 3 & 22.75 & 1.000 & 2.000 & 1.500 & 2.150 & 16.06 & 16.19 & 13.54 & 5850 & 69.00 & 51.99 & 537 & 4696 \\
\hline 7.1 & 2 & 16.00 & 1.000 & 2.000 & 4.000 & 2.120 & 16.03 & 16.20 & 13.58 & 5240 & 69.00 & 45.40 & 495 & 4215 \\
\hline
\end{tabular}




\section{B.2 EVALUATION DATA SET}

\begin{tabular}{|c|c|c|c|c|c|c|c|c|c|c|c|c|c|c|}
\hline Test No. & $\mathrm{n}$ & $\begin{array}{c}l_{d} \\
\text { (in.) }\end{array}$ & $\begin{array}{c}\mathrm{d}_{\mathrm{b}} \\
\text { (in.) }\end{array}$ & $\begin{array}{c}\mathrm{c}_{\mathrm{so}} \\
\text { (in.) }\end{array}$ & $\begin{array}{c}\mathrm{c}_{\mathbf{d}} \\
\text { (in.) }\end{array}$ & $\begin{array}{c}c_{b} \\
\text { (in.) }\end{array}$ & $\begin{array}{c}\mathrm{b} \\
\text { (in.) }\end{array}$ & $\begin{array}{c}\mathrm{h} \\
\text { (in.) }\end{array}$ & $\begin{array}{c}\mathrm{d} \\
\text { (in.) }\end{array}$ & $\begin{array}{c}\mathrm{f}_{\mathrm{c}} \\
\text { (psi) }\end{array}$ & $\begin{array}{c}f_{y} \\
(k s i)\end{array}$ & $\begin{array}{c}\mathrm{f}_{s} * \\
\text { (ksi) }\end{array}$ & $\begin{array}{l}\mathrm{T} d \mathrm{f}_{\mathrm{c}}{ }^{1 / 2} \\
\left(\mathrm{in}{ }^{2}{ }^{2}\right)\end{array}$ & $\begin{array}{l}T_{c} / f_{c}{ }^{1 / 4} \\
\left(\text { in. }^{2}\right)\end{array}$ \\
\hline \multicolumn{15}{|c|}{ Zuo (1998) } \\
\hline $25.1 * *$ & 3 & 16.50 & 0.625 & 1.985 & 1.023 & 1.556 & 12.19 & 16.27 & 14.37 & 4490 & 62.98 & 63.72 & 295 & 2413 \\
\hline 19.1 & 3 & 36.00 & 1.000 & 1.953 & 1.930 & 1.961 & 18.14 & 16.16 & 13.66 & 4250 & 80.57 & 73.51 & 891 & 7193 \\
\hline 19.2 & 3 & 36.00 & 1.000 & 2.016 & 1.883 & 1.929 & 18.06 & 16.13 & 13.66 & 4250 & 80.57 & 67.85 & 822 & 6639 \\
\hline 20.6 & 3 & 40.00 & 1.000 & 1.516 & 0.672 & 1.300 & 12.08 & 15.60 & 13.76 & 5080 & 80.57 & 57.15 & 633 & 5348 \\
\hline $23 a .5$ & 2 & 22.00 & 1.000 & 2.000 & 1.891 & 1.938 & 18.19 & 16.16 & 13.63 & 9320 & 80.57 & 62.24 & 509 & 5005 \\
\hline $23 a .6$ & 2 & 29.00 & 1.000 & 2.031 & 1.875 & 1.919 & 12.24 & 16.11 & 13.67 & 9320 & 80.57 & 75.47 & 618 & 6068 \\
\hline $23 b .3$ & 2 & 19.50 & 1.000 & 3.031 & 3.859 & 3.057 & 18.23 & 16.32 & 12.72 & 8370 & 80.57 & 71.64 & 619 & 5917 \\
\hline 24.1 & 2 & 32.00 & 1.000 & 2.000 & 1.875 & 1.903 & 12.14 & 16.12 & 13.69 & 4300 & 79.70 & 61.91 & 746 & 6040 \\
\hline 26.3 & 3 & 40.00 & 1.000 & 1.547 & 0.652 & 1.889 & 12.11 & 16.19 & 13.78 & 4960 & 79.70 & 62.52 & 701 & 5885 \\
\hline 26.5 & 3 & 40.00 & 1.000 & 1.500 & 0.684 & 1.891 & 12.15 & 16.17 & 13.75 & 4960 & 77.96 & 64.36 & 722 & 6058 \\
\hline 31.5 & 3 & 22.00 & 1.000 & 1.828 & 0.508 & 1.494 & 12.26 & 15.58 & 13.56 & 12890 & 79.70 & 61.43 & 427 & 4555 \\
\hline 31.6 & 3 & 22.00 & 1.000 & 1.719 & 0.539 & 1.492 & 12.17 & 15.49 & 13.44 & 12890 & 69.50 & 63.42 & 441 & 4702 \\
\hline 34.1 & 3 & 24.00 & 1.000 & 2.063 & 1.938 & 1.941 & 18.13 & 16.12 & 13.66 & 5440 & 79.70 & 57.88 & 620 & 5324 \\
\hline 34.2 & 3 & 24.00 & 1.000 & 2.070 & 1.945 & 1.918 & 18.17 & 16.05 & 13.61 & 5440 & 79.70 & 61.97 & 664 & 5701 \\
\hline 34.3 & 3 & 24.00 & 1.000 & 2.080 & 1.844 & 1.981 & 18.12 & 16.02 & 13.49 & 5440 & 69.50 & 58.94 & 631 & 5422 \\
\hline 34.4 & 3 & 24.00 & 1.000 & 2.045 & 1.883 & 1.936 & 18.21 & 16.02 & 13.53 & 5440 & 69.50 & 58.49 & 626 & 5380 \\
\hline 36.3 & 3 & 26.00 & 1.000 & 2.016 & 1.836 & 2.000 & 18.17 & 16.10 & 13.55 & 5060 & 69.50 & 62.78 & 697 & 5881 \\
\hline 36.4 & 3 & 26.00 & 1.000 & 2.031 & 1.828 & 1.988 & 18.14 & 16.10 & 13.56 & 5060 & 69.50 & 60.17 & 668 & 5636 \\
\hline 38.1 & 3 & 26.00 & 1.000 & 1.938 & 1.953 & 1.802 & 18.25 & 16.10 & 13.75 & 5080 & 69.50 & 53.96 & 598 & 5049 \\
\hline 38.2 & 3 & 26.00 & 1.000 & 2.125 & 1.844 & 2.075 & 18.17 & 16.14 & 13.51 & 5080 & 69.50 & 60.30 & 668 & 5643 \\
\hline 39.6 & 3 & 21.00 & 1.000 & 1.953 & 0.516 & 1.505 & 12.19 & 15.41 & 13.59 & 14450 & 67.69 & 67.38 & 443 & 4855 \\
\hline 40.5 & 2 & 17.00 & 1.000 & 2.000 & 1.875 & 1.846 & 12.11 & 16.04 & 13.67 & 15650 & 77.96 & 65.81 & 416 & 4649 \\
\hline 28.5 & 2 & 30.00 & 1.410 & 1.977 & 4.031 & 1.999 & $18.09^{\circ}$ & 16.20 & 13.45 & 12610 & 77.77 & 50.89 & 707 & 7492 \\
\hline 30.5 & 2 & 30.00 & 1.410 & 2.063 & 4.016 & 1.956 & 18.12 & 16.15 & 13.44 & 13220 & 77.77 & 66.95 & 908 & 9740 \\
\hline 32.1 & 2 & 32.00 & 1.410 & 2.000 & 0.984 & 1.904 & 12.17 & 16.17 & 13.52 & 14400 & 77.77 & 63.33 & 823 & 9019 \\
\hline 32.2 & 2 & 32.00 & 1.410 & 2.000 & 1.063 & 1.916 & 12.14 & 16.16 & 13.51 & 14400 & 66.69 & 61.49 & 799 & 8757 \\
\hline 32.3 & 2 & 32.00 & 1.410 & 1.969 & 4.016 & 1.947 & 18.14 & 16.15 & 13.45 & 14400 & 77.77 & 60.64 & 788 & 8635 \\
\hline 32.4 & 2 & 28.00 & 1.410 & 2.031 & 4.047 & 1.935 & 18.20 & 16.17 & 13.50 & 14400 & 66.69 & 61.01 & 793 & 8688 \\
\hline
\end{tabular}




\begin{tabular}{|c|c|c|c|c|c|c|c|c|c|}
\hline 39 & -40 & 20.3 & 2.18 & 0.086 & 0.08 & 0.108 & 0.1 & 0.886 & 34.88 \\
\hline 24 & -40 & 20.3 & 1.73 & 0.068 & 0.08 & 0.085 & 0.1 & 2.411 & 94.92 \\
\hline 5 & -40 & 20.3 & 1.98 & 0.078 & 0.08 & 0.098 & 0.1 & 2.17 & 85.43 \\
\hline 13 & -68.9 & 20.3 & 1.37 & 0.054 & 0.08 & 0.068 & 0.1 & 0.358 & 14.09 \\
\hline 19 & -68.9 & 20.3 & 2.06 & 0.081 & 0.08 & 0.101 & 0.1 & 0.525 & 20.67 \\
\hline 22 & -68.9 & 20.3 & 1.91 & 0.075 & 0.08 & 0.094 & 0.1 & 0.617 & 24.29 \\
\hline 2 & -103.9 & 20.3 & 1.96 & 0.077 & 0.08 & 0.096 & 0.1 & 0.251 & 9.88 \\
\hline 21 & -103.9 & 20.3 & 2.24 & 0.088 & 0.08 & 0.11 & 0.1 & 0.251 & 9.88 \\
\hline 28 & -103.9 & 20.3 & 1.91 & 0.075 & 0.08 & 0.094 & 0.1 & 0.213 & 8.39 \\
\hline 29 & -103.9 & 20.3 & 1.91 & 0.075 & 0.08 & 0.094 & 0.1 & 0.523 & 20.59 \\
\hline 26 & -195.6 & 20.3 & 2.24 & 0.088 & 0.08 & 0.11 & 0.1 & 0.005 & 0.2 \\
\hline 3 & -195.6 & 20.3 & 2.69 & 0.106 & 0.08 & 0.133 & 0.1 & 0.005 & 0.2 \\
\hline 65 & 21.1 & 31.8 & 10.44 & 0.411 & 0.4 & 0.329 & 0.32 & 0.708 & 27.87 \\
\hline 66 & 21.1 & 31.8 & 10.36 & 0.408 & 0.4 & 0.326 & 0.32 & 1.368 & 53.86 \\
\hline 69 & 21.1 & 31.8 & 9.88 & 0.389 & 0.4 & 0.311 & 0.32 & 1.31 & 51.57 \\
\hline 72 & 21.1 & 31.8 & 10.11 & 0.398 & 0.4 & 0.318 & 0.32 & 1.173 & 46.18 \\
\hline 67 & -5.6 & 31.8 & 10.18 & 0.401 & 0.4 & 0.321 & 0.32 & 0.368 & 14.49 \\
\hline 68 & -5.6 & 31.8 & 10.24 & 0.403 & 0.4 & 0.322 & 0.32 & 0.632 & 24.88 \\
\hline 71 & -5.6 & 31.8 & 9.8 & 0.386 & 0.4 & 0.309 & 0.32 & 0.383 & 15.08 \\
\hline \multicolumn{10}{|c|}{ Fracture mechanics } \\
\hline 3 & -36 & 100 & 10 & 0.394 & 0.4 & 0.1 & 0.1 & 0.586 & 23.07 \\
\hline 7 & -59 & 94 & 10.2 & 0.402 & 0.4 & 0.109 & 0.1 & 0.137 & 5.39 \\
\hline 8 & -60 & 94 & 9.6 & 0.378 & 0.4 & 0.102 & 0.1 & 0.476 & 18.74 \\
\hline 9 & -62 & 94 & 9.5 & 0.374 & 0.4 & 0.101 & 0.1 & 0.352 & 13.86 \\
\hline 10 & -60 & 94 & 14 & 0.551 & 0.4 & 0.149 & 0.1 & 0.235 & 9.25 \\
\hline 11 & -57 & 94 & 8.4 & 0.331 & 0.4 & 0.089 & 0.1 & 0.196 & 7.72 \\
\hline 13 & -60 & 94 & 8.8 & 0.346 & 0.4 & 0.094 & 0.1 & 0.357 & 14.06 \\
\hline 14 & -60 & 93 & 8.7 & 0.343 & 0.4 & 0.094 & 0.1 & 0.346 & 13.62 \\
\hline 15 & -59 & 94 & 8.7 & 0.343 & 0.4 & 0.093 & 0.1 & 0.146 & 5.75 \\
\hline 18 & -24 & 102 & 10.6 & 0.417 & 0.4 & 0.104 & 0.1 & 0.468 & 18.43 \\
\hline 20 & -4 & 101 & 10.8 & 0.425 & 0.4 & 0.107 & 0.1 & 1.733 & 68.23 \\
\hline 21 & -23 & 102 & 10.7 & 0.421 & 0.4 & 0.105 & 0.1 & 0.306 & 12.05 \\
\hline 22 & -7 & 102 & 10.9 & 0.429 & 0.4 & 0.107 & 0.1 & 0.942 & 37.09 \\
\hline 26 & -40 & 102 & 11 & 0.433 & 0.4 & 0.108 & 0.1 & 0.355 & 13.98 \\
\hline 27 & -22 & 102 & 10.7 & 0.421 & 0.4 & 0.105 & 0.1 & 0.559 & 22.01 \\
\hline 28 & -6 & 102 & 10.3 & 0.406 & 0.4 & 0.101 & 0.1 & 1.242 & 48.9 \\
\hline 32 & -103 & 102 & 11.1 & 0.437 & 0.4 & 0.109 & 0.1 & 0.016 & 0.63 \\
\hline 33 & -103 & 102 & 10.7 & 0.421 & 0.4 & 0.105 & 0.1 & 0.009 & 0.35 \\
\hline 34 & -106 & 102 & 10.4 & 0.409 & 0.4 & 0.102 & 0.1 & 0.017 & 0.67 \\
\hline 37 & -39 & 102 & 10.8 & 0.425 & 0.4 & 0.106 & 0.1 & 0.263 & 10.35 \\
\hline 38 & -39 & 102 & 10.8 & 0.425 & 0.4 & 0.106 & 0.1 & 0.206 & 8.11 \\
\hline
\end{tabular}




\section{APPENDIX D}

\section{THE DATA SET USED IN SECTION 5.4}

\begin{tabular}{|c|c|c|c|c|c|c|}
\hline $\begin{array}{l}\text { solubility } \\
\left(\times 10^{5} \mathrm{M}\right)\end{array}$ & pKal & compound & particle_size & medium $\mathrm{pH}$ & $\begin{array}{l}\text { flowrate } \\
(\mathrm{ml} / \mathrm{min})\end{array}$ & $\begin{array}{c}\text { flux } \\
\left(\times 10^{10} \mathrm{~mol} / \mathrm{min} . \mathrm{cm}^{2}\right\}\end{array}$ \\
\hline 13.7 & 4.02 & acidic & 120 & 2 & 5.1 & 24.38 \\
\hline 13.7 & 4.02 & acidic & 120 & 3 & 5.1 & 28.73 \\
\hline 13.7 & 4.02 & acidic & 120 & 4 & 5.1 & 45.27 \\
\hline 13.7 & 4.02 & acidic & 120 & 6 & 5.1 & 87.06 \\
\hline 13.7 & 4.02 & acidic & 120 & 7 & 5.1 & 95.77 \\
\hline 13.7 & 4.02 & acidic & 120 & 8 & 5.1 & 104.47 \\
\hline 13.7 & 4.02 & acidic & 120 & 9 & 5.1 & 128.85 \\
\hline 13.7 & 4.02 & acidic & 120 & 10 & 5.1 & 430.95 \\
\hline 2150 & 4.03 & acidic & 120 & 2 & 1.1 & 2989.52 \\
\hline 2150 & 4.03 & acidic & 120 & 2 & 3.48 & 4442.16 \\
\hline 2150 & 4.03 & acidic & 120 & 2 & 5.1 & 5356.48 \\
\hline 2150 & 4.03 & acidic & 120 & 2 & 8.49 & 5785.91 \\
\hline 2150 & 4.03 & acidic & 120 & 2 & 13.41 & 6969.39 \\
\hline 2150 & 4.03 & acidic & 120 & 2 & 5.1 & 5356.78 \\
\hline 2150 & 4.03 & acidic & 120 & 4 & 5.1 & 5356.78 \\
\hline 2150 & 4.03 & acidic & 120 & 7 & 5.1 & 5503.54 \\
\hline 2150 & 4.03 & acidic & 120 & 9 & 5.1 & 5503.54 \\
\hline 2150 & 4.03 & acidic & 120 & 10.6 & 5.1 & 6237.34 \\
\hline 2150 & 4.03 & acidic & 120 & 11.2 & 5.1 & 7117.91 \\
\hline 2150 & 4.03 & acidic & 120 & 12 & 5.1 & 11887.64 \\
\hline 2150 & 4.03 & acidic & 120 & 12.2 & 5.1 & 19959.49 \\
\hline 2150 & 4.03 & acidic & 120 & 12.5 & 5.1 & 33167.98 \\
\hline 13.7 & 4.57 & acidic & 137 & 2 & 5 & 21.43 \\
\hline 13.7 & 4.57 & acidic & 137 & 2 & 5 & 23.6 \\
\hline 13.7 & 4.57 & acidic & 37 & 7 & 5 & 144.8 \\
\hline 13.7 & 4.57 & acidic & 137 & 2 & 5 & 22.29 \\
\hline 13.7 & 4.57 & acidic & 37 & 7 & 5 & 138.3 \\
\hline 13.7 & 4.57 & acidic & 137 & 7 & 5 & 133.5 \\
\hline 13.7 & 4.57 & acidic & 111.5 & 7 & 5 & 138.3 \\
\hline
\end{tabular}




\section{APPENDIX E}

\section{THE DATA SET USED IN SECTION 5.5}

\begin{tabular}{|c|c|c|c|c|c|c|c|c|}
\hline$m p g$ & c & d & $\mathrm{h}$ & w & a & $y$ & 0 & Car Name \\
\hline 18 & 8 & 307 & 130 & 3504 & 12 & 70 & 1 & chevrolet chevelle malibu \\
\hline 15 & 8 & 350 & 165 & 3693 & 11.5 & 70 & 1 & buick skylark 320 \\
\hline 18 & 8 & 318 & 150 & 3436 & 11 & 70 & 1 & plymouth satellite \\
\hline 16 & 8 & 304 & 150 & 3433 & 12 & 70 & 1 & amc rebel sst \\
\hline 17 & 8 & 302 & 140 & 3449 & 10.5 & 70 & 1 & ford torino \\
\hline 15 & 8 & 429 & 198 & 4341 & 10 & 70 & 1 & ford galaxie 500 \\
\hline 14 & 8 & 454 & 220 & 4354 & 9 & 70 & 1 & chevrolet impala \\
\hline 14 & 8 & 440 & 215 & 4312 & 8.5 & 70 & 1 & plymouth fury iii \\
\hline 14 & 8 & 455 & 225 & 4425 & 10 & 70 & 1 & pontiac catalina \\
\hline 15 & 8 & 390 & 190 & 3850 & 8.5 & 70 & 1 & amc ambassador dpl \\
\hline 15 & 8 & 383 & 170 & 3563 & 10 & 70 & 1 & dodge challenger se \\
\hline 14 & 8 & 340 & 160 & 3609 & 8 & 70 & 1 & plymouth 'cuda 340 \\
\hline 15 & 8 & 400 & 150 & 3761 & 9.5 & 70 & 1 & chevrolet monte carlo \\
\hline 14 & 8 & 455 & 225 & 3086 & 10 & 70 & 1 & buick estate wagon (sw) \\
\hline 24 & 4 & 113 & 95 & 2372 & 15 & 70 & 3 & toyota corona mark ii \\
\hline 22 & 6 & 198 & 95 & 2833 & 15.5 & 70 & 1 & plymouth duster \\
\hline 18 & 6 & 199 & 97 & 2774 & 15.5 & 70 & 1 & anc hornet \\
\hline 21 & 6 & 200 & 85 & 2587 & 16 & 70 & 1 & ford maverick \\
\hline 27 & 4 & 97 & 88 & 2130 & 14.5 & 70 & 3 & datsun pl510 \\
\hline 26 & 4 & 97 & 46 & 1835 & 20.5 & 70 & 2 & volkswagen 1131 deluxe sedan \\
\hline 25 & 4 & 110 & 87 & 2672 & 17.5 & 70 & 2 & peugeot 504 \\
\hline 24 & 4 & 107 & 90 & 2430 & 14.5 & 70 & 2 & audi $100 \mathrm{ls}$ \\
\hline 25 & 4 & 104 & 95 & 2375 & 17.5 & 70 & 2 & saab $99 e$ \\
\hline 26 & 4 & 121 & 113 & 2234 & 12.5 & 70 & 2 & bmw 2002 \\
\hline 21 & 6 & 199 & 90 & 2648 & 15 & 70 & 1 & ame gremlin \\
\hline 10 & 8 & 360 & 215 & 4615 & 14 & 70 & 1 & ford $\mathrm{f} 250$ \\
\hline 10 & 8 & 307 & 200 & 4376 & 15 & 70 & 1 & chevy c20 \\
\hline 11 & 8 & 318 & 210 & 4382 & 13.5 & 70 & 1 & dodge d200 \\
\hline 9 & 8 & 304 & 193 & 4732 & 18.5 & 70 & 1 & hs $1200 \mathrm{~d}$ \\
\hline
\end{tabular}




\begin{tabular}{|c|c|c|c|c|c|c|c|c|}
\hline 15 & 8 & 304 & 150 & 3892 & 12.5 & 72 & 1 & amc matador (sw) \\
\hline 13 & 8 & 307 & 130 & 4098 & 14 & 72 & 1 & chevrolet chevelle concours (sw) \\
\hline 13 & 8 & 302 & 140 & 4294 & 16 & 72 & 1 & ford gran torino (sw) \\
\hline 14 & 8 & 318 & 150 & 4077 & 14 & 72 & 1 & plymouth satellite custom (sw) \\
\hline 18 & 4 & 121 & 112 & 2933 & 14.5 & 72 & 2 & volvo $145 \mathrm{e}$ (sw) \\
\hline 22 & 4 & 121 & 76 & 2511 & 18 & 72 & 2 & volkswagen 411 (sw) \\
\hline 21 & 4 & 120 & 87 & 2979 & 19.5 & 72 & 2 & peugeot 504 (sw) \\
\hline 26 & 4 & 96 & 69 & 2189 & 18 & 72 & 2 & renault $12(\mathrm{sw})$ \\
\hline 22 & 4 & 122 & 86 & 2395 & 16 & 72 & 1 & ford pinto (sw) \\
\hline 28 & 4 & 97 & 92 & 2288 & 17 & 72 & 3 & datsun $510(\mathrm{sw})$ \\
\hline 23 & 4 & 120 & 97 & 2506 & 14.5 & 72 & 3 & toyouta corona mark ii (sw) \\
\hline 28 & 4 & 98 & 80 & 2164 & 15 & 72 & 1 & dodge colt (sw) \\
\hline 27 & 4 & 97 & 88 & 2100 & 16.5 & 72 & 3 & toyota corolla 1600 (sw) \\
\hline 13 & 8 & 350 & 175 & 4100 & 13 & 73 & 1 & buick century 350 \\
\hline 14 & 8 & 304 & 150 & 3672 & 11.5 & 73 & 1 & amc matador \\
\hline 13 & 8 & 350 & 145 & 3988 & 13 & 73 & 1 & chevrolet malibu \\
\hline 14 & 8 & 302 & 137 & 4042 & 14.5 & 73 & 1 & ford gran torino \\
\hline 15 & 8 & 318 & 150 & 3777 & 12.5 & 73 & 1 & dodge coronet custom \\
\hline 12 & 8 & 429 & 198 & 4952 & 11.5 & 73 & 1 & mercury marquis brougham \\
\hline 13 & 8 & 400 & 150 & 4464 & 12 & 73 & 1 & chevrolet caprice classic \\
\hline 13 & 8 & 351 & 158 & 4363 & 13 & 73 & 1 & ford ltd \\
\hline 14 & 8 & 318 & 150 & 4237 & 14.5 & 73 & 1 & plymouth fury gran sedan \\
\hline 13 & 8 & 440 & 215 & 4735 & 11 & 73 & 1 & chrysler new yorker brougham \\
\hline 12 & 8 & 455 & 225 & 4951 & 11 & 73 & 1 & buick electra 225 custom \\
\hline 13 & 8 & 360 & 175 & 3821 & 11 & 73 & 1 & amc ambassador brougham \\
\hline 18 & 6 & 225 & 105 & 3121 & 16.5 & 73 & 1 & plymouth valiant \\
\hline 16 & 6 & 250 & 100 & 3278 & 18 & 73 & 1 & chevrolet nova custom \\
\hline 18 & 6 & 232 & 100 & 2945 & 16 & 73 & 1 & amc hornet \\
\hline 18 & 6 & 250 & 88 & 3021 & 16.5 & 73 & 1 & ford maverick \\
\hline 23 & 6 & 198 & 95 & 2904 & 16 & 73 & 1 & plymouth duster \\
\hline 26 & 4 & 97 & 46 & 1950 & 21 & 73 & 2 & volkswagen super beetle \\
\hline 11 & 8 & 400 & 150 & 4997 & 14 & 73 & 1 & chevrolet impala \\
\hline 12 & 8 & 400 & 167 & 4906 & 12.5 & 73 & 1 & ford country \\
\hline 13 & 8 & 360 & 170 & 4654 & 13 & 73 & 1 & plymouth custom suburb \\
\hline 12 & 8 & 350 & 180 & 4499 & 12.5 & 73 & 1 & oldsmobile vista cruiser \\
\hline 18 & 6 & 232 & 100 & 2789 & 15 & 73 & 1 & ame gremlin \\
\hline 20 & 4 & 97 & 88 & 2279 & 19 & 73 & 3 & toyota carina \\
\hline 21 & 4 & 140 & 72 & 2401 & 19.5 & 73 & 1 & chevrolet vega \\
\hline 22 & 4 & 108 & 94 & 2379 & 16.5 & 73 & 3 & datsun 610 \\
\hline 18 & 3 & 70 & 90 & 2124 & 13.5 & 73 & 3 & $\operatorname{maxda} r \times 3$ \\
\hline 19 & 4 & 122 & 85 & 2310 & 18.5 & 73 & 1 & ford pinto \\
\hline 21 & 6 & 155 & 107 & 2472 & 14 & 73 & 1 & mercury capri v6 \\
\hline 26 & 4 & 98 & 90 & 2265 & 15.5 & 73 & 2 & fiat 124 sport coupe \\
\hline
\end{tabular}




\begin{tabular}{|c|c|c|c|c|c|c|c|c|}
\hline 16 & 8 & 318 & 150 & 4498 & 14.5 & 75 & 1 & plymouth grand fury \\
\hline 14 & 8 & 351 & 148 & 4657 & 13.5 & 75 & 1 & ford ltd \\
\hline 17 & 6 & 231 & 110 & 3907 & 21 & 75 & 1 & buick century \\
\hline 16 & 6 & 250 & 105 & 3897 & 18.5 & 75 & 1 & chevroelt chevelle malibu \\
\hline 15 & 6 & 258 & 110 & 3730 & 19 & 75 & 1 & amc matador \\
\hline 18 & 6 & 225 & 95 & 3785 & 19 & 75 & 1 & plymouth fury \\
\hline 21 & 6 & 231 & 110 & 3039 & 15 & 75 & 1 & buick skyhawk \\
\hline 20 & 8 & 262 & 110 & 3221 & 13.5 & 75 & 1 & chevrolet monza $2+2$ \\
\hline 13 & 8 & 302 & 129 & 3169 & 12 & 75 & 1 & ford mustang ii \\
\hline 29 & 4 & 97 & 75 & 2171 & 16 & 75 & 3 & toyota corolla \\
\hline 23 & 4 & 140 & 83 & 2639 & 17 & 75 & 1 & ford pinto \\
\hline 20 & 6 & 232 & 100 & 2914 & 16 & 75 & 1 & amc gremlin \\
\hline 23 & 4 & 140 & 78 & 2592 & 18.5 & 75 & 1 & pontiac astro \\
\hline 24 & 4 & 134 & 96 & 2702 & 13.5 & 75 & 3 & toyota corona \\
\hline 25 & 4 & 90 & 71 & 2223 & 16.5 & 75 & 2 & volkswagen dasher \\
\hline 24 & 4 & 119 & 97 & 2545 & 17 & 75 & 3 & datsun 710 \\
\hline 18 & 6 & 171 & 97 & 2984 & 14.5 & 75 & 1 & ford pinto \\
\hline 29 & 4 & 90 & 70 & 1937 & 14 & 75 & 2 & volkswagen rabbit \\
\hline 19 & 6 & 232 & 90 & 3211 & 17 & 75 & 1 & amc pacer \\
\hline 23 & 4 & 115 & 95 & 2694 & 15 & 75 & 2 & audi $1001 \mathrm{~s}$ \\
\hline 23 & 4 & 120 & 88 & 2957 & 17 & 75 & 2 & peugeot 504 \\
\hline 22 & 4 & 121 & 98 & 2945 & 14.5 & 75 & 2 & volvo $244 \mathrm{dl}$ \\
\hline 25 & 4 & 121 & 115 & 2671 & 13.5 & 75 & 2 & saab 991e \\
\hline 33 & 4 & 91 & 53 & 1795 & 17.5 & 75 & 3 & honda civic crec \\
\hline 28 & 4 & 107 & 86 & 2464 & 15.5 & 76 & 2 & fiat 131 \\
\hline 25 & 4 & 116 & 81 & 2220 & 16.9 & 76 & 2 & opel 1900 \\
\hline 25 & 4 & 140 & 92 & 2572 & 14.9 & 76 & 1 & capri ii \\
\hline 26 & 4 & 98 & 79 & 2255 & 17.7 & 76 & 1 & dodge colt \\
\hline 27 & 4 & 101 & 83 & 2202 & 15.3 & 76 & 2 & renault 12 tl \\
\hline 17.5 & 8 & 305 & 140 & 4215 & 13 & 76 & 1 & chevrolet cheveile malibu classic \\
\hline 16 & 8 & 318 & 150 & 4190 & 13 & 76 & 1 & dodge coronet brougham \\
\hline 15.5 & 8 & 304 & 120 & 3962 & 13.9 & 76 & 1 & amc matador \\
\hline 14.5 & 8 & 351 & 152 & 4215 & 12.8 & 76 & 1 & ford gran torino \\
\hline 22 & 6 & 225 & 100 & 3233 & 15.4 & 76 & 1 & plymouth valiant \\
\hline 22 & 6 & 250 & 105 & 3353 & 14.5 & 76 & 1 & chevrolet nova \\
\hline 24 & 6 & 200 & 81 & 3012 & 17.6 & 76 & 1 & ford maverick \\
\hline 22.5 & 6 & 232 & 90 & 3085 & 17.6 & 76 & 1 & amc hornet \\
\hline 29 & 4 & 85 & 52 & 2035 & 22.2 & 76 & 1 & chevrolet chevette \\
\hline 24.5 & 4 & 98 & 60 & 2164 & 22.1 & 76 & 1 & chevrolet woody \\
\hline 29 & 4 & 90 & 70 & 1937 & 14.2 & 76 & 2 & vw rabbit \\
\hline 33 & 4 & 91 & 53 & 1795 & 17.4 & 76 & 3 & honda civic \\
\hline 20 & 6 & 225 & 100 & 3651 & 17.7 & 76 & 1 & dodge aspen se \\
\hline 18 & 6 & 250 & 78 & 3574 & 21 & 76 & 1 & ford granada ghia \\
\hline
\end{tabular}




\begin{tabular}{|c|c|c|c|c|c|c|c|c|}
\hline 43.1 & 4 & 90 & 48 & 1985 & 21.5 & 78 & 2 & volkswagen rabbit custom diesel \\
\hline 36.1 & 4 & 98 & 66 & 1800 & 14.4 & 78 & 1 & ford fiesta \\
\hline 32.8 & 4 & 78 & 52 & 1985 & 19.4 & 78 & 3 & mazda glc deluxe \\
\hline 39.4 & 4 & 85 & 70 & 2070 & 18.6 & 78 & 3 & datsun $\mathrm{b} 210 \mathrm{gx}$ \\
\hline 36.1 & 4 & 91 & 60 & 1800 & 16.4 & 78 & 3 & honda civic evcc \\
\hline 19.9 & 8 & 260 & 110 & 3365 & 15.5 & 78 & 1 & oldsmobile cutlass salon brougham \\
\hline 19.4 & 8 & 318 & 140 & 3735 & 13.2 & 78 & 1 & dodge diplomat \\
\hline 20.2 & 8 & 302 & 139 & 3570 & 12.8 & 78 & 1 & mercury monarch ghia \\
\hline 19.2 & 6 & 231 & 105 & 3535 & 19.2 & 78 & 1 & pontiac phoenix lj \\
\hline 20.5 & 6 & 200 & 95 & 3155 & 18.2 & 78 & 1 & chevrolet malibu \\
\hline 20.2 & 6 & 200 & 85 & 2965 & 15.8 & 78 & 1 & ford fairmont (auto) \\
\hline 25.1 & 4 & 140 & 88 & 2720 & 15.4 & 78 & 1 & ford fairmont (man) \\
\hline 20.5 & 6 & 225 & 100 & 3430 & 17.2 & 78 & 1 & plymouth volare \\
\hline 19.4 & 6 & 232 & 90 & 3210 & 17.2 & 78 & 1 & ame concord \\
\hline 20.6 & 6 & 231 & 105 & 3380 & 15.8 & 78 & 1 & buick century special \\
\hline 20.8 & 6 & 200 & 85 & 3070 & 16.7 & 78 & 1 & mercury zephyr \\
\hline 18.6 & 6 & 225 & 110 & 3620 & 18.7 & 78 & 1 & dodge aspen \\
\hline 18.1 & 6 & 258 & 120 & 3410 & 15.1 & 78 & 1 & ame concord $d / l$ \\
\hline 19.2 & 8 & 305 & 145 & 3425 & 13.2 & 78 & 1 & chevrolet monte carlo landau \\
\hline 17.7 & 6 & 231 & 165 & 3445 & 13.4 & 78 & 1 & buick regal sport coupe (turbo) \\
\hline 18.1 & 8 & 302 & 139 & 3205 & 11.2 & 78 & 1 & ford futura \\
\hline 17.5 & 8 & 318 & 140 & 4080 & 13.7 & 78 & 1 & dodge magnum xe \\
\hline 30 & 4 & 98 & 68 & 2155 & 16.5 & 78 & 1 & chevrolet chevette \\
\hline 27.5 & 4 & 134 & 95 & 2560 & 14.2 & 78 & 3 & toyota corona \\
\hline 27.2 & 4 & 119 & 97 & 2300 & 14.7 & 78 & 3 & datsun 510 \\
\hline 30.9 & 4 & 105 & 75 & 2230 & 14.5 & 78 & 1 & dodge omni \\
\hline 21.1 & 4 & 134 & 95 & 2515 & 14.8 & 78 & 3 & toyota celica gt liftback \\
\hline 23.2 & 4 & 156 & 105 & 2745 & 16.7 & 78 & 1 & plymouth sapporo \\
\hline 23.8 & 4 & 151 & 85 & 2855 & 17.6 & 78 & 1 & oldsmobile starfire sx \\
\hline 23.9 & 4 & 119 & 97 & 2405 & 14.9 & 78 & 3 & datsun 200-sx \\
\hline 20.3 & 5 & 131 & 103 & 2830 & 15.9 & 78 & 2 & audi 5000 \\
\hline 17 & 6 & 163 & 125 & 3140 & 13.6 & 78 & 2 & volvo $264 \mathrm{gl}$ \\
\hline 21.6 & 4 & 121 & 115 & 2795 & 15.7 & 78 & 2 & saab 99gle \\
\hline 16.2 & 6 & 163 & 133 & 3410 & 15.8 & 78 & 2 & peugeot $604 s i$ \\
\hline 31.5 & 4 & 89 & 71 & 1990 & 14.9 & 78 & 2 & volkswagen scirocco \\
\hline 29.5 & 4 & 98 & 68 & 2135 & 16.6 & 78 & 3 & honda accord ix \\
\hline 21.5 & 6 & 231 & 115 & 3245 & 15.4 & 79 & 1 & pontiac lemans v6 \\
\hline 19.8 & 6 & 200 & 85 & 2990 & 18.2 & 79 & 1 & mercury zephyr 6 \\
\hline 22.3 & 4 & 140 & 88 & 2890 & 17.3 & 79 & 1 & ford fairmont 4 \\
\hline 20.2 & 6 & 232 & 90 & 3265 & 18.2 & 79 & 1 & amc concord dl 6 \\
\hline 20.6 & 6 & 225 & 110 & 3360 & 16.6 & 79 & 1 & dodge aspen 6 \\
\hline 17 & 8 & 305 & 130 & 3840 & 15.4 & 79 & 1 & chevrolet caprice classic \\
\hline 17.6 & 8 & 302 & 129 & 3725 & 13.4 & 79 & 1 & ford ltd landau \\
\hline
\end{tabular}




\begin{tabular}{|c|c|c|c|c|c|c|c|c|}
\hline 40.9 & 4 & 85 & $?$ & 1835 & 17.3 & 80 & 2 & renault lecar deluxe \\
\hline 33.8 & 4 & 97 & 67 & 2145 & 18 & 80 & 3 & subaru dl \\
\hline 29.8 & 4 & 89 & 62 & 1845 & 15.3 & 80 & 2 & vokswagen rabbit \\
\hline 32.7 & 6 & 168 & 132 & 2910 & 11.4 & 80 & 3 & datsun $280-z x$ \\
\hline 23.7 & 3 & 70 & 100 & 2420 & 12.5 & 80 & 3 & mazda rx-7 gs \\
\hline 35 & 4 & 122 & 88 & 2500 & 15.1 & 80 & 2 & triumph tr 7 coupe \\
\hline 23.6 & 4 & 140 & $?$ & 2905 & 14.3 & 80 & 1 & ford mustang cobra \\
\hline 32.4 & 4 & 107 & 72 & 2290 & 17 & 80 & 3 & honda accord \\
\hline 27.2 & 4 & 135 & 84 & 2490 & 15.7 & 81 & 1 & plymouth reliant \\
\hline 26.6 & 4 & 151 & 84 & 2635 & 16.4 & 81 & 1 & buick skylark \\
\hline 25.8 & 4 & 156 & 92 & 2620 & 14.4 & 81 & 1 & dodge aries wagon (sw) \\
\hline 23.5 & 6 & 173 & 110 & 2725 & 12.6 & 81 & 1 & chevrolet citation \\
\hline 30 & 4 & 135 & 84 & 2385 & 12.9 & 81 & 1 & plymouth reliant \\
\hline 39.1 & 4 & 79 & 58 & 1755 & 16.9 & 81 & 3 & toyota starlet \\
\hline 39 & 4 & 86 & 64 & 1875 & 16.4 & 81 & 1 & plymouth champ \\
\hline 35.1 & 4 & 81 & 60 & 1760 & 16.1 & 81 & 3 & honda civic 1300 \\
\hline 32.3 & 4 & 97 & 67 & 2065 & 17.8 & 81 & 3 & subaru \\
\hline 37 & 4 & 85 & 65 & 1975 & 19.4 & 81 & 3 & datsun $210 \mathrm{mpg}$ \\
\hline 37.7 & 4 & 89 & 62 & 2050 & 17.3 & 81 & 3 & toyota tercel \\
\hline 34.1 & 4 & 91 & 68 & 1985 & 16 & 81 & 3 & mazda glc 4 \\
\hline 34.7 & 4 & 105 & 63 & 2215 & 14.9 & 81 & 1 & plymouth horizon 4 \\
\hline 34.4 & 4 & 98 & 65 & 2045 & 16.2 & 81 & 1 & ford escort 4w \\
\hline 29.9 & 4 & 98 & 65 & 2380 & 20.7 & 81 & 1 & ford escort $2 \mathrm{~h}$ \\
\hline 33 & 4 & 105 & 74 & 2190 & 14.2 & 81 & 2 & volkswagen jetta \\
\hline 34.5 & 4 & 100 & $?$ & 2320 & 15.8 & 81 & 2 & renault $18 \mathrm{i}$ \\
\hline 33.7 & 4 & 107 & 75 & 2210 & 14.4 & 81 & 3 & honda prelude \\
\hline 32.4 & 4 & 108 & 75 & 2350 & 16.8 & 81 & 3 & toyota corolla \\
\hline 32.9 & 4 & 119 & 100 & 2615 & 14.8 & 81 & 3 & datsun 200 sx \\
\hline 31.6 & 4 & 120 & 74 & 2635 & 18.3 & 81 & 3 & mazda 626 \\
\hline 28.1 & 4 & 141 & 80 & 3230 & 20.4 & 81 & 2 & peugeot 505 s turbo diesel \\
\hline 30.7 & 6 & 145 & 76 & 3160 & 19.6 & 81 & 2 & volvo diesel \\
\hline 25.4 & 6 & 168 & 116 & 2900 & 12.6 & 81 & 3 & toyota cressida \\
\hline 24.2 & 6 & 146 & 120 & 2930 & 13.8 & 81 & 3 & datsun 810 maxima \\
\hline 22.4 & 6 & 231 & 110 & 3415 & 15.8 & 81 & 1 & buick century \\
\hline 26.6 & 8 & 350 & 105 & 3725 & 19 & 81 & 1 & oldsmobile cutlass is \\
\hline 20.2 & 6 & 200 & 88 & 3060 & 17.1 & 81 & 1 & ford granada gl \\
\hline 17.6 & 6 & 225 & 85 & 3465 & 16.6 & 81 & 1 & chrysler lebaron salon \\
\hline 28 & 4 & 112 & 88 & 2605 & 19.6 & 82 & 1 & chevrolet cavalier \\
\hline 27 & 4 & 112 & 88 & 2640 & 18.6 & 82 & 1 & chevrolet cavalier wagon \\
\hline 34 & 4 & 112 & 88 & 2395 & 18 & 82 & 1 & chevrolet cavalier 2-door \\
\hline 31 & 4 & 112 & 85 & 2575 & 16.2 & 82 & 1 & pontiac j2000 se hatchback \\
\hline 29 & 4 & 135 & 84 & 2525 & 16 & 82 & 1 & dodge aries se \\
\hline 27 & 4 & 151 & 90 & 2735 & 18 & 82 & 1 & pontiac phoenix \\
\hline
\end{tabular}

